\title{
Structuring Peptide Dendrimers Through pH Modulation and Substrate Binding
}

Luís C. S. Filipe, ${ }^{\dagger}$ Sara R. R. Campos, ${ }^{\dagger}$ Miguel Machuqueiro, ${ }^{\ddagger}$ Tamis Darbre, ${ }^{\mathbb{I I}}$ and António M. Baptista ${ }^{*,+}$

†Instituto de Tecnologia Química e Biológica, Universidade Nova de Lisboa, Av. da República, EAN, 2780-157 Oeiras, Portugal

$\ddagger$ Centro de Química e Bioquímica and Departamento de Química e Bioquímica, Faculdade de Ciências, Universidade de Lisboa, 1749-016 Lisboa, Portugal

IIDepartment of Chemistry and Biochemistry, University of Bern, Freiestrasse 3, 3012 Bern, Switzerland

E-mail: baptista@itqb.unl.pt

Phone: +351-21-4469619. Fax: +351-21-4411277

Supporting Information 


\section{Contents}

1 Equilibration times $\quad$ S3

2 Replicate sampling convergence $\quad$ S28

$3 \quad$ Parameterization of substrate BPTS $\quad$ S33

4 Dendrimer-substrate equilibration times $\quad$ S41

5 Dendrimer-substrate replicate sampling convergence S44

$\begin{array}{lll}\mathrm{X}-\mathrm{H} \cdots \pi \text { test } & \text { S45 }\end{array}$

$\begin{array}{lll}7 & \text { A1 and A2 titration curves } & \text { S46 }\end{array}$

$8 R_{\mathrm{g}}$ histograms at different $\mathrm{pH}$ values $\quad S 49$

9 Average SASA at different $\mathrm{pH}$ values $\quad$ S50

10 Energy landscapes: A1, A2 and A3 S51

$11 \pi-\pi$ interactions $\quad$ S54

12 Hydrogen Bonds: MainChain vs. SideChain $S 55$

13 Histidines protonation curves $\quad$ S56

14 Dendrimer-Substrate contact area $\quad S 64$

15 Dendrimer-substrate interaction tests $\quad$ S65

16 Dendrimer-Substrate complexes $\quad$ S66

$\begin{array}{ll}\text { References } & \text { S72 }\end{array}$ 


\section{Equilibration times}




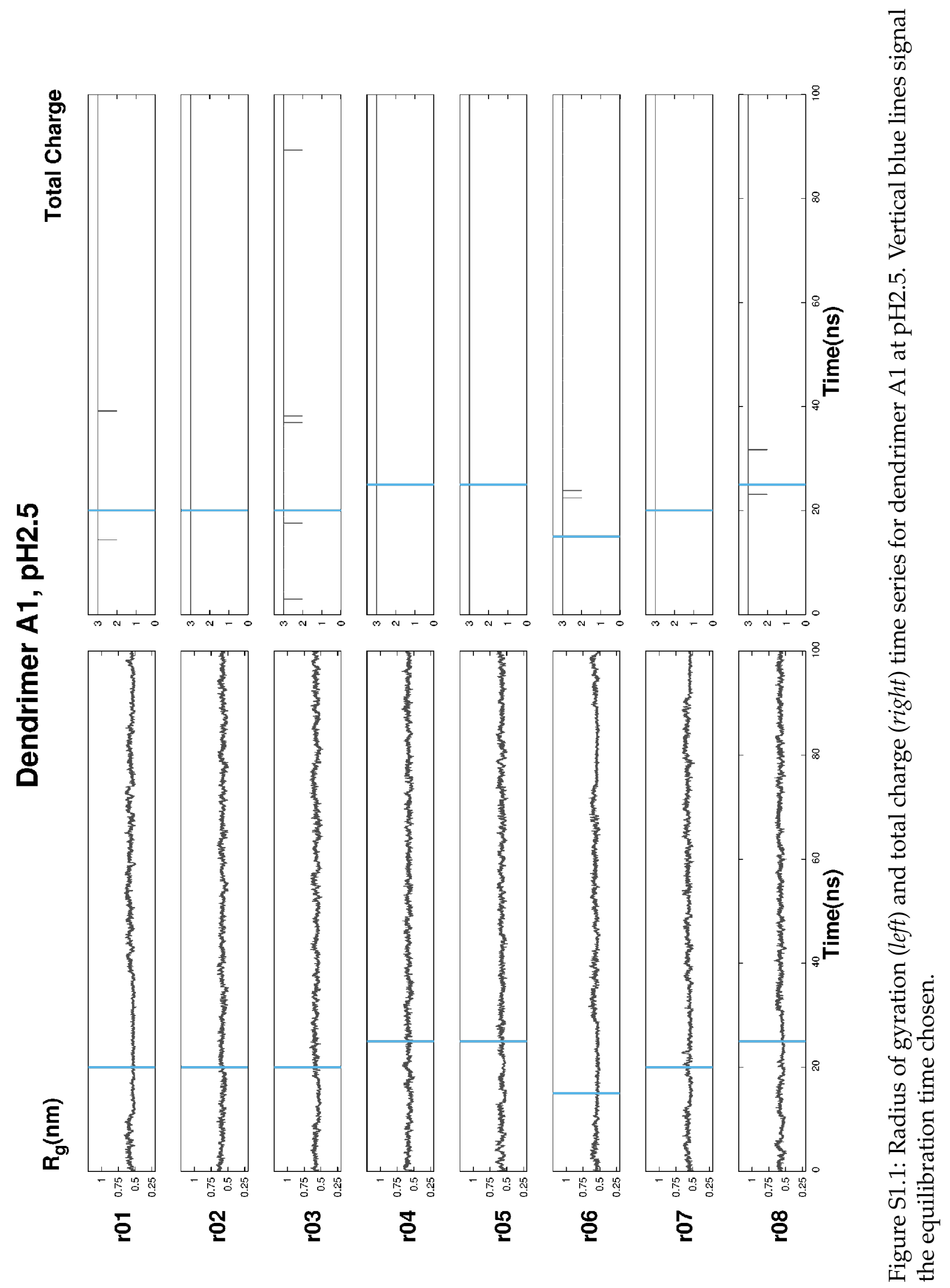




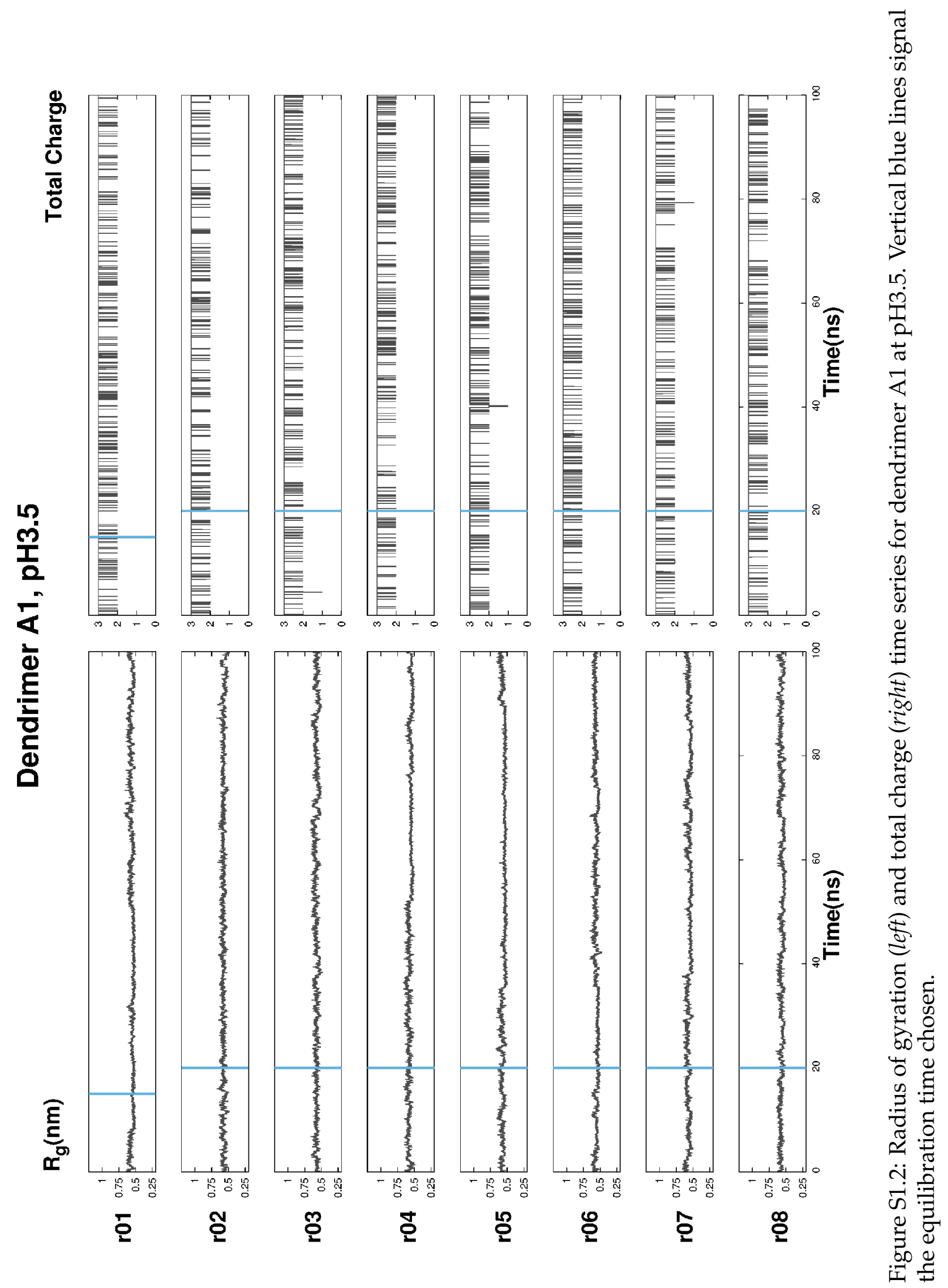




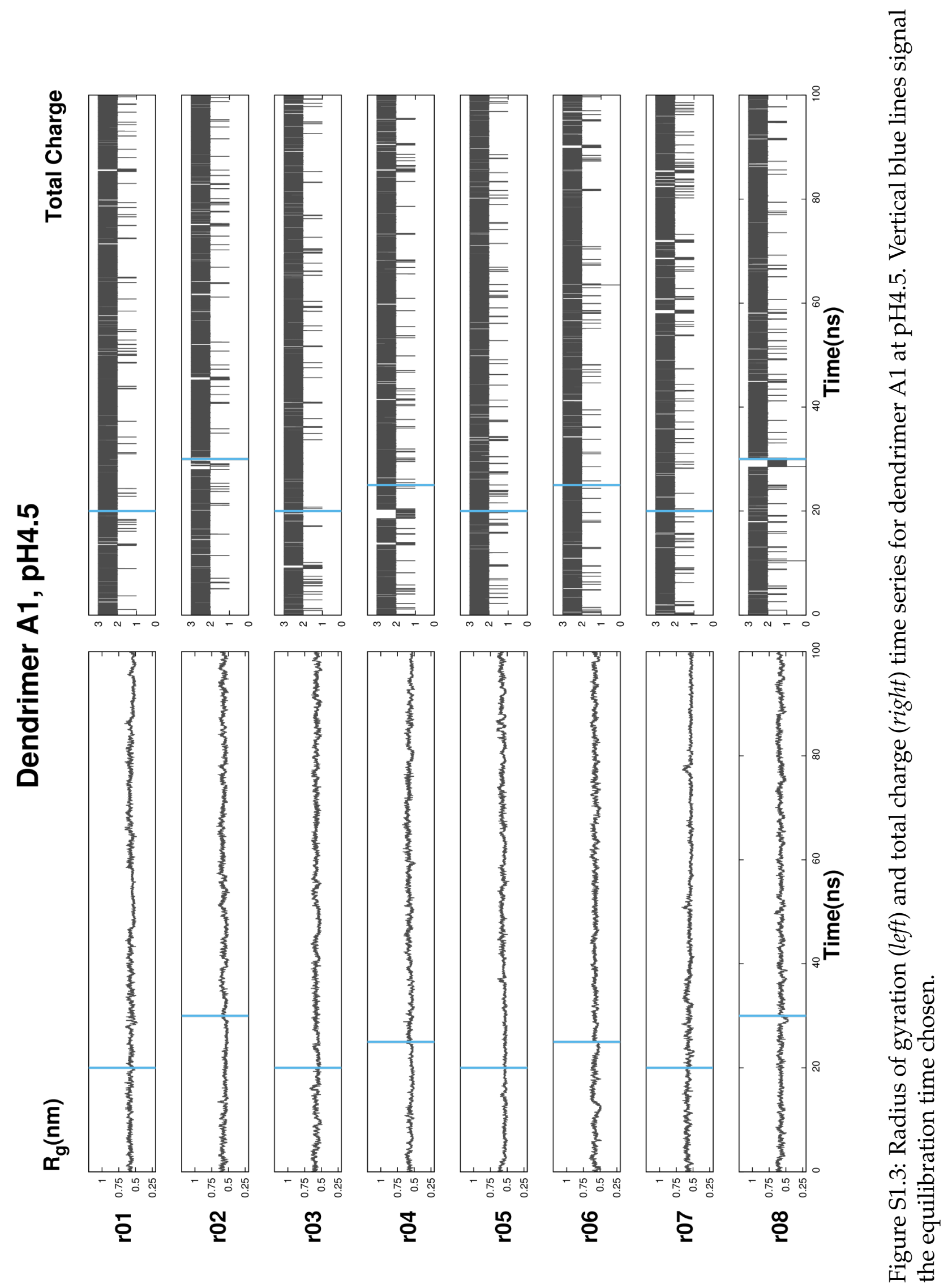




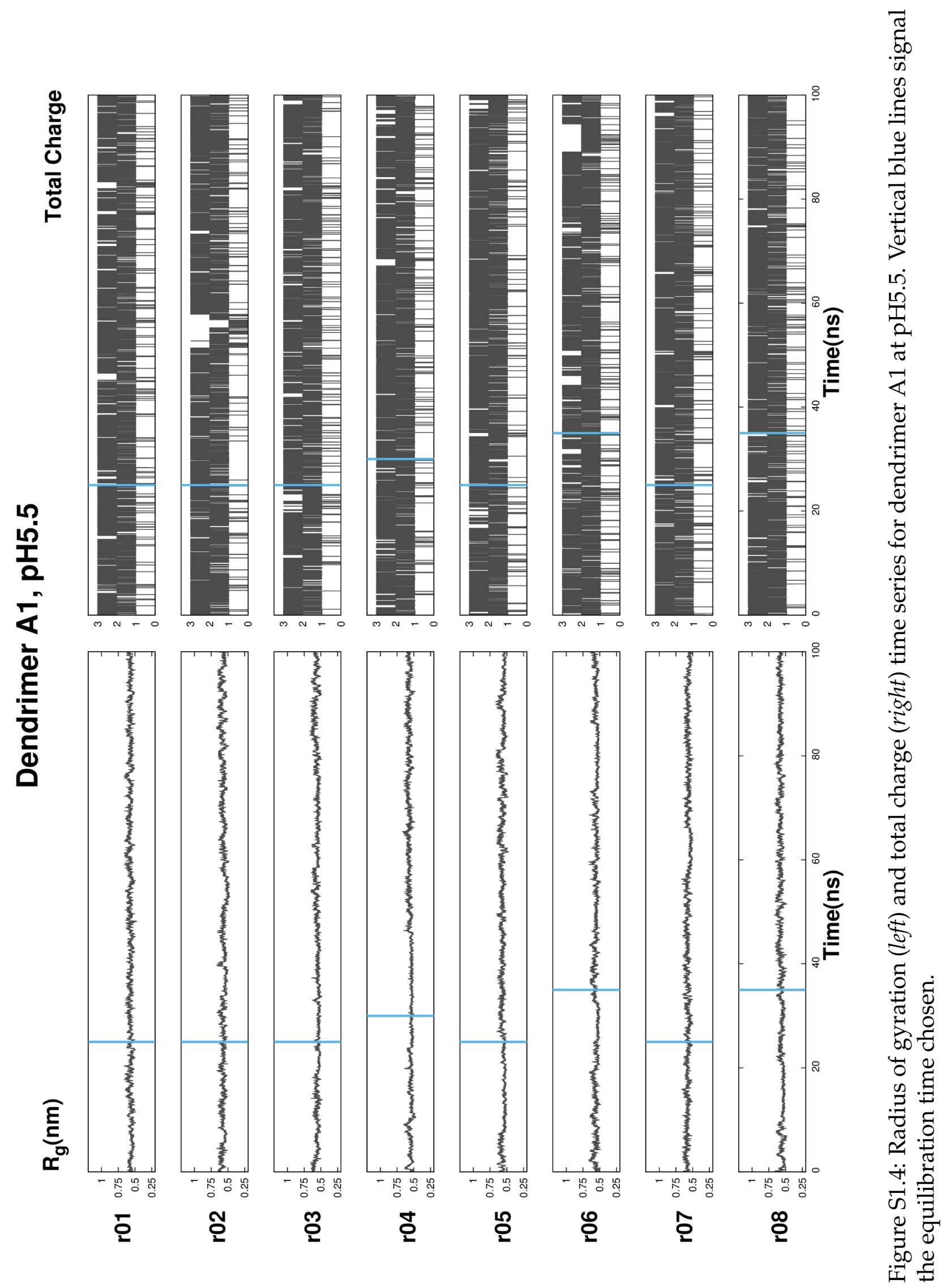




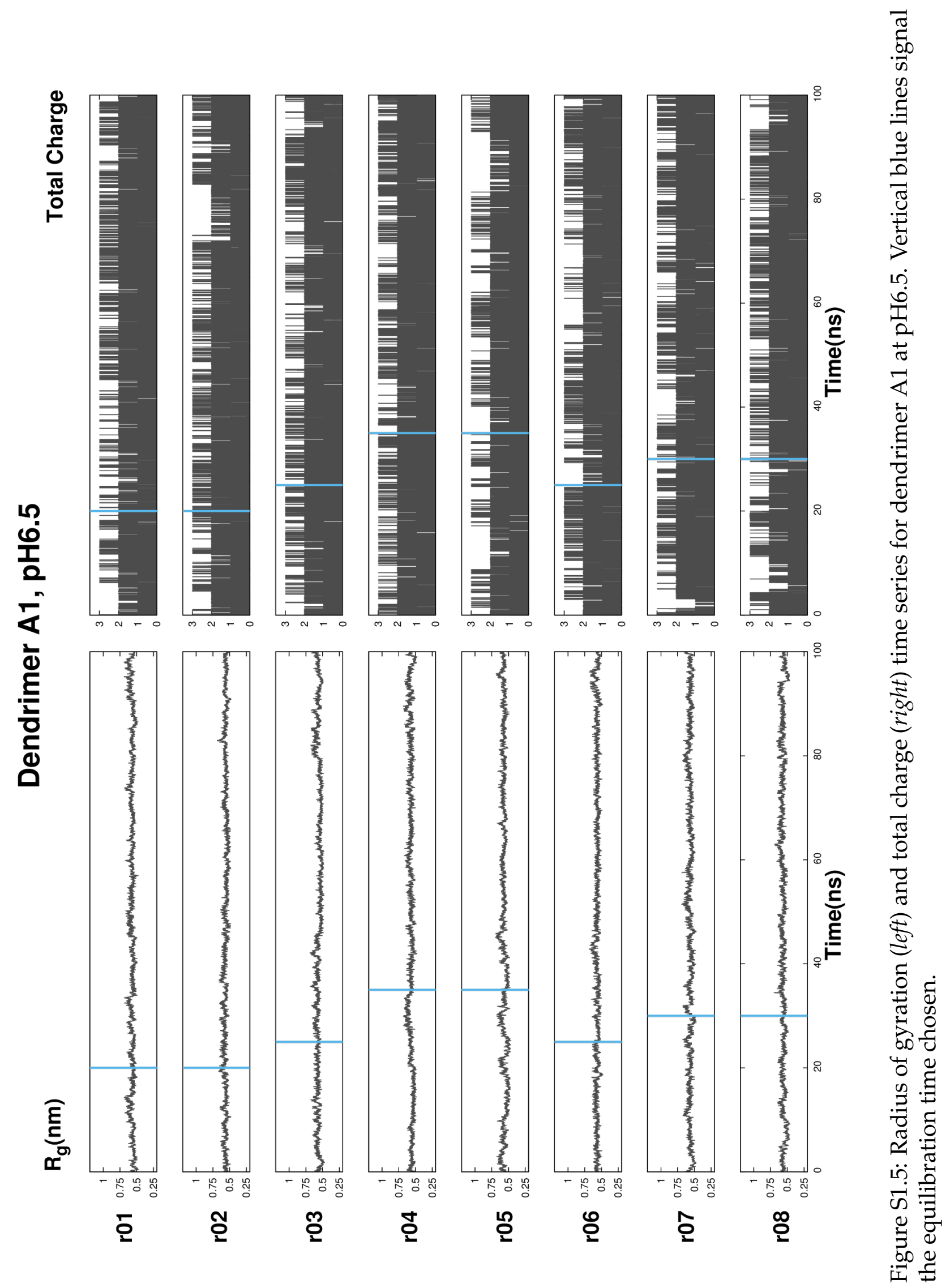




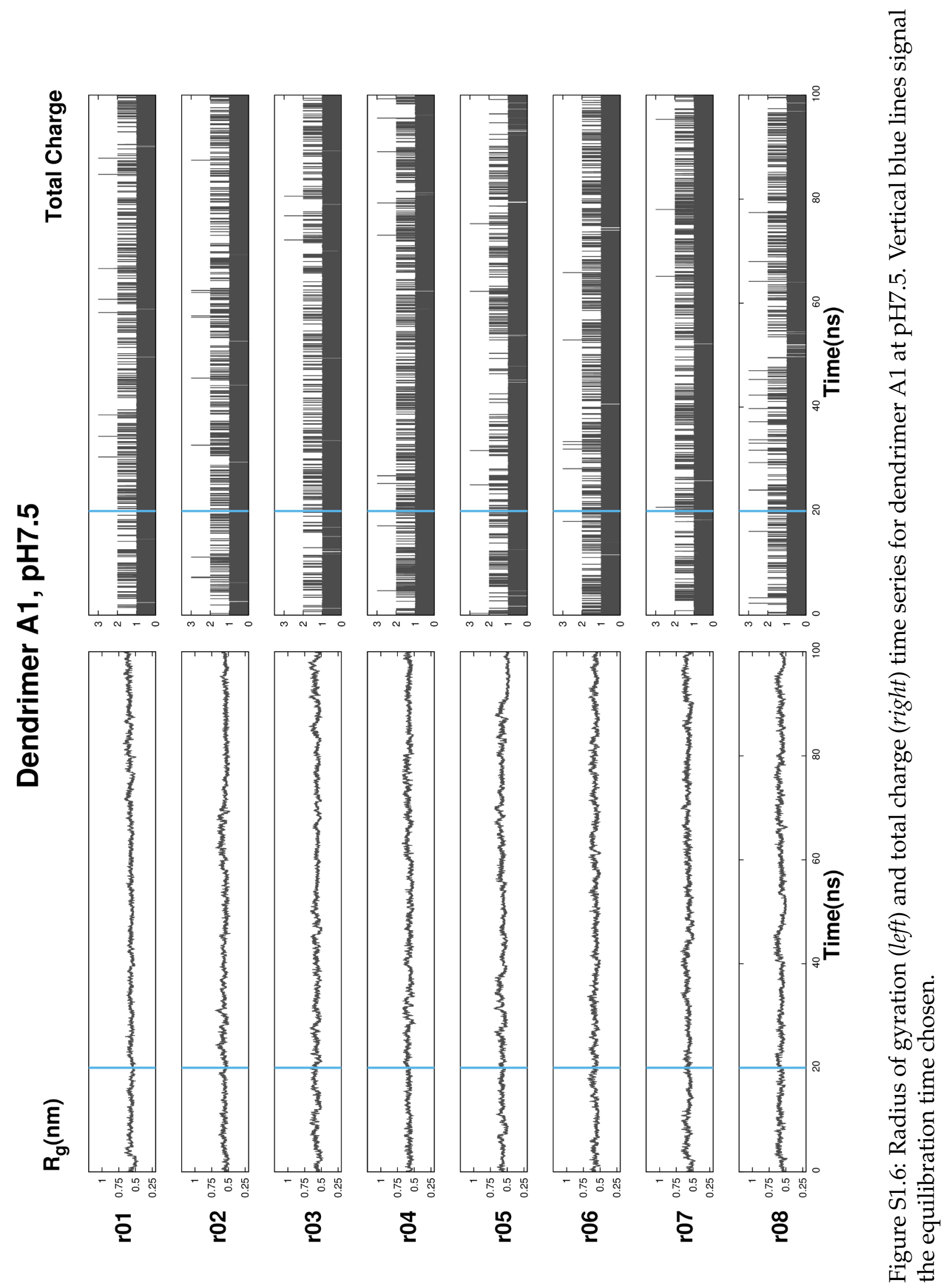




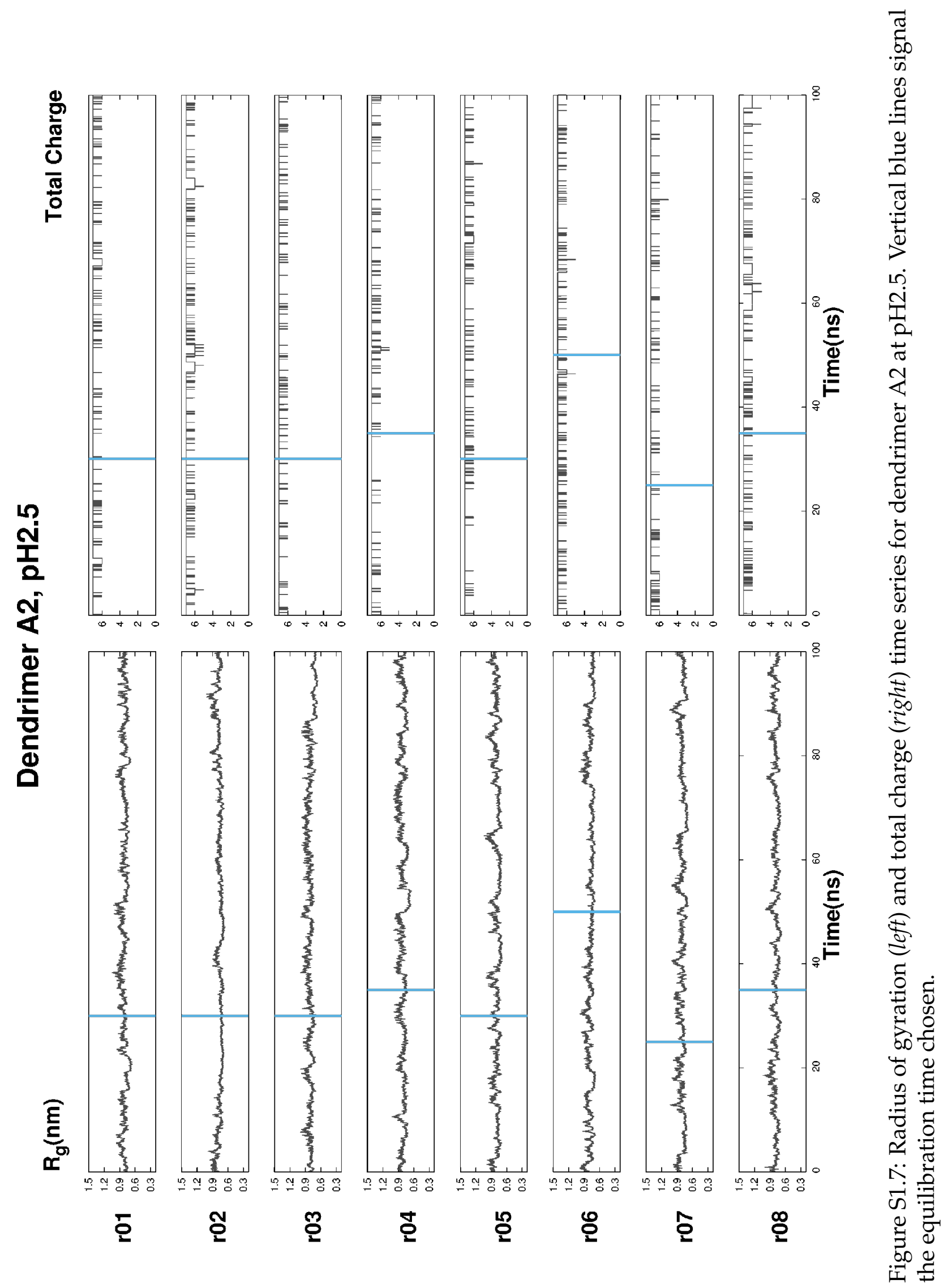




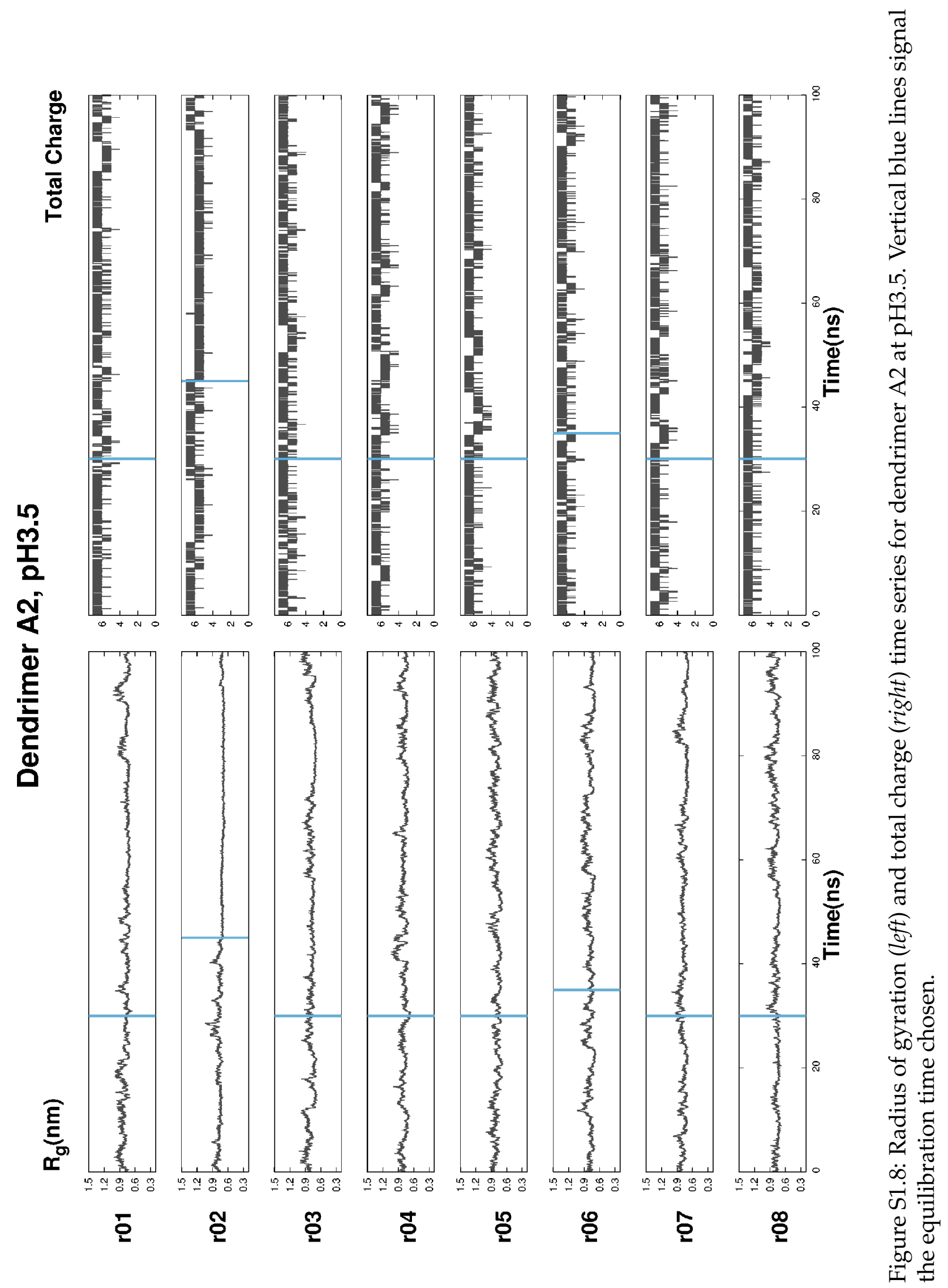




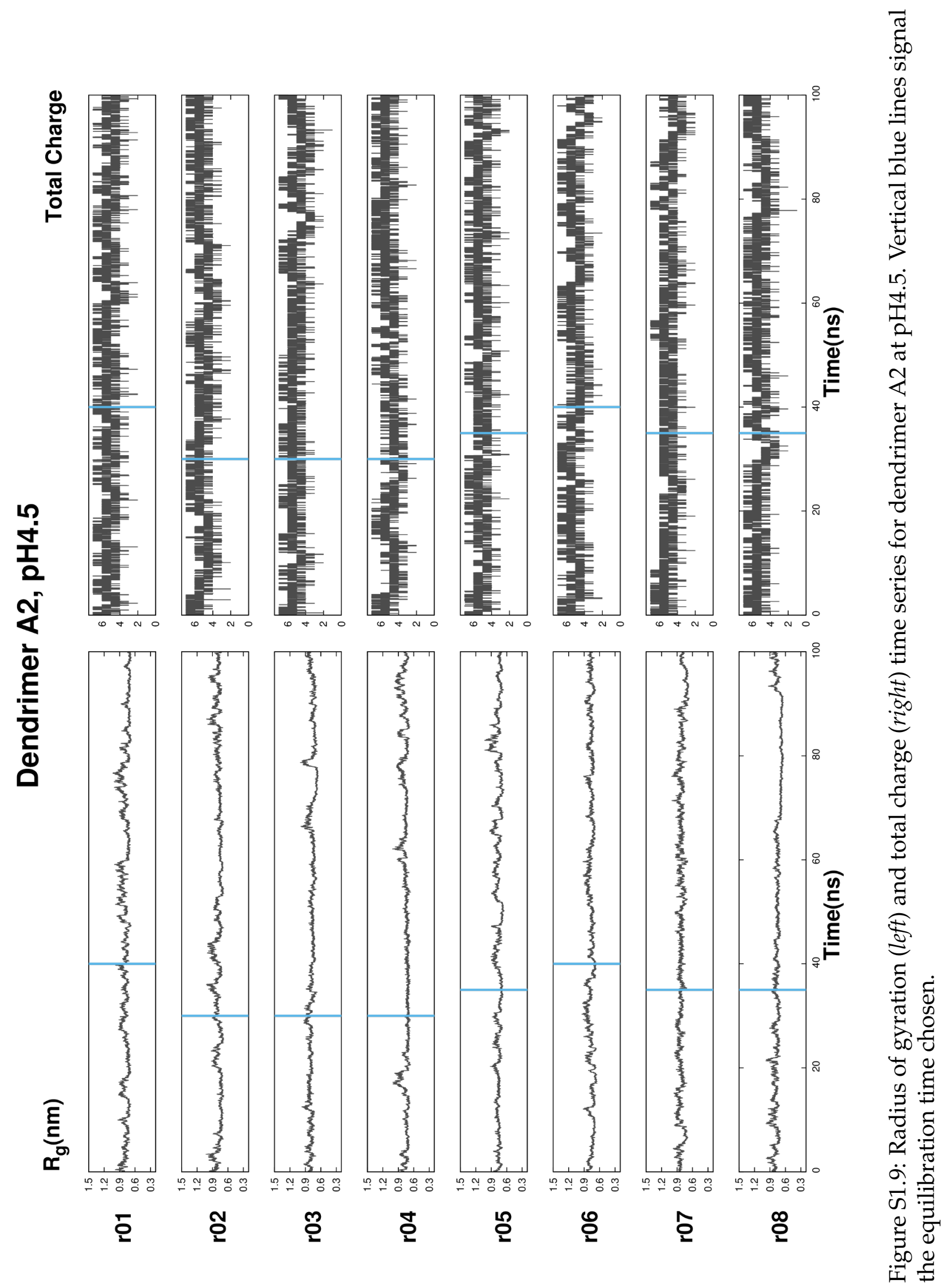




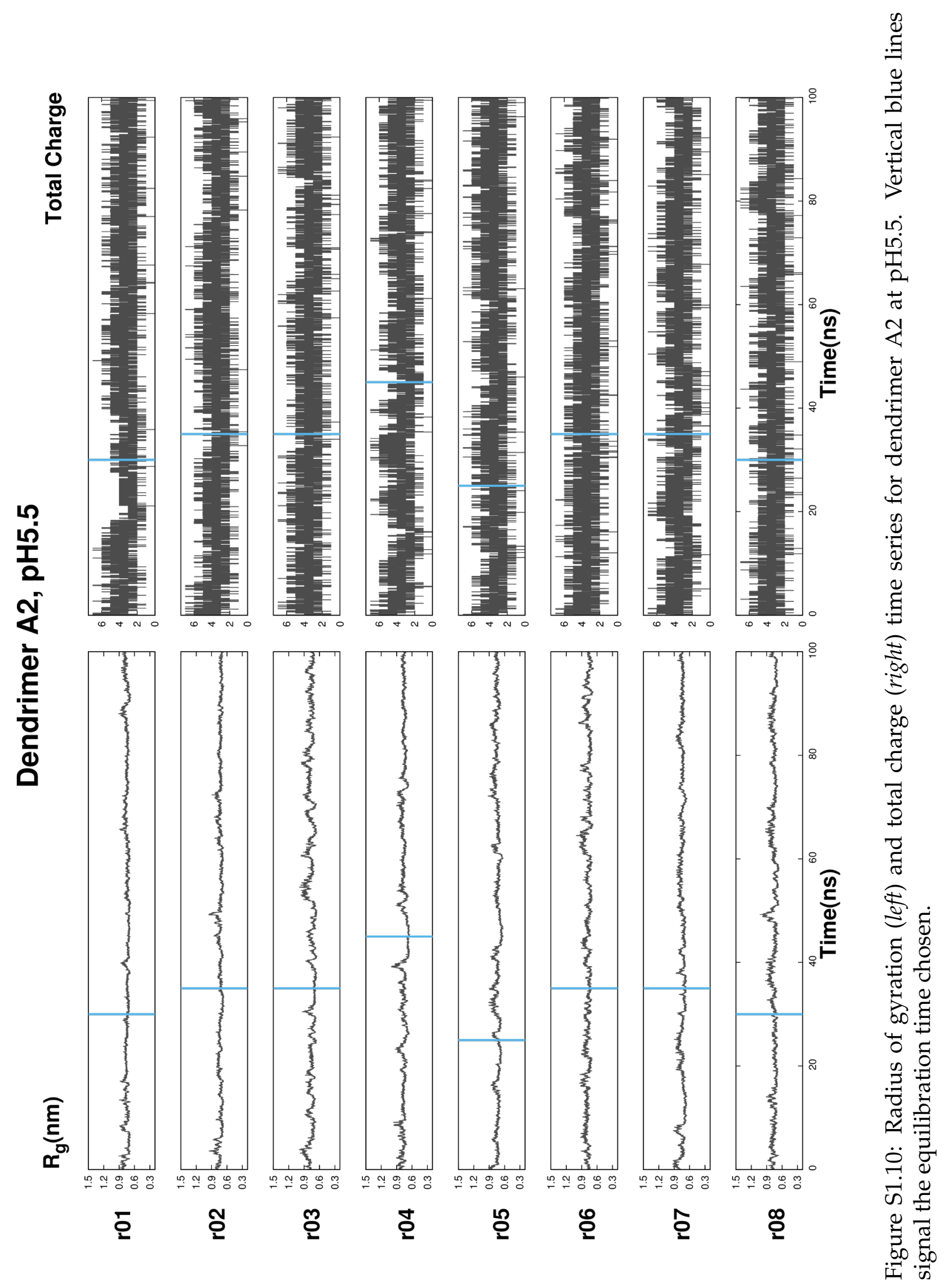




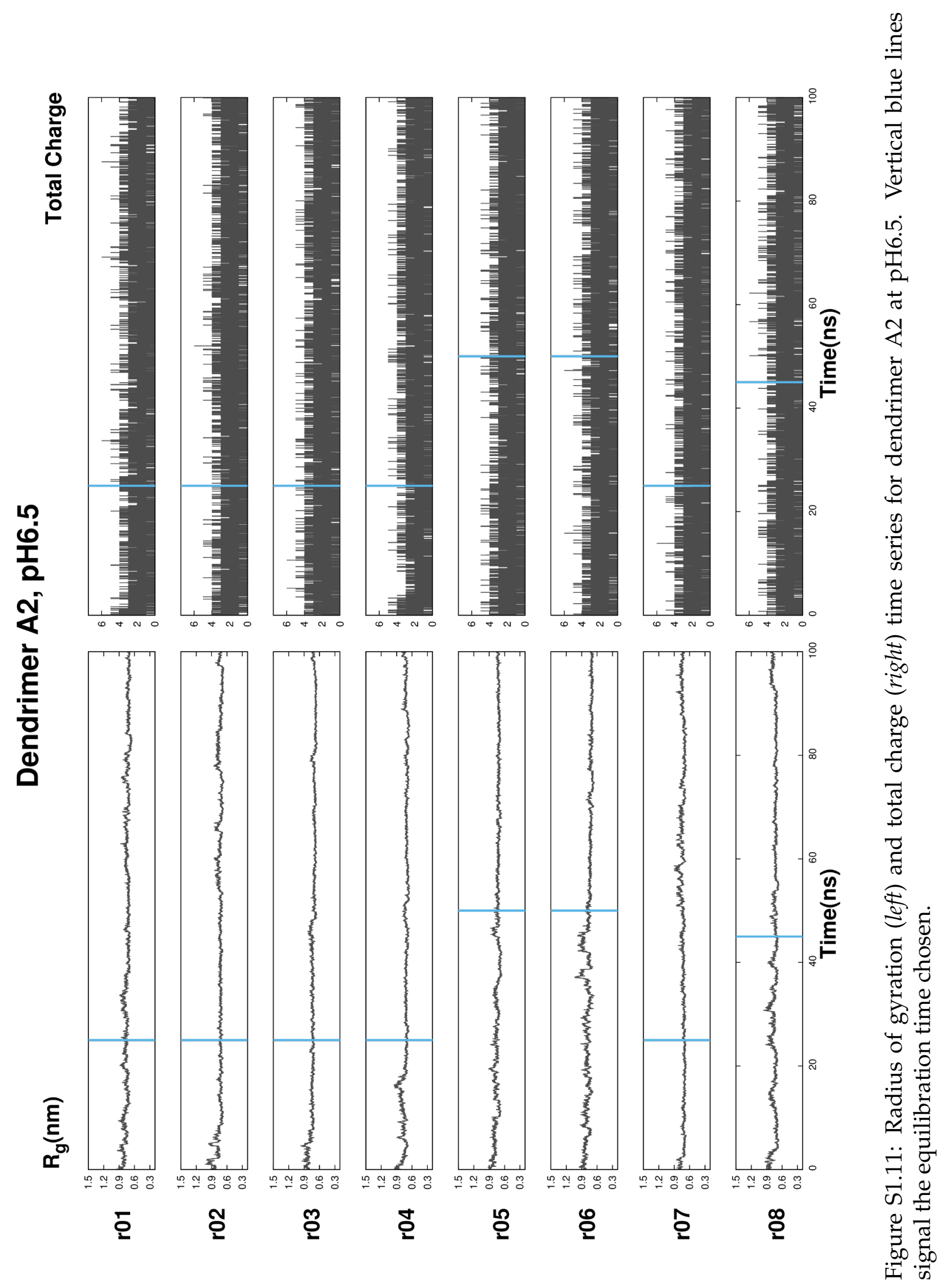




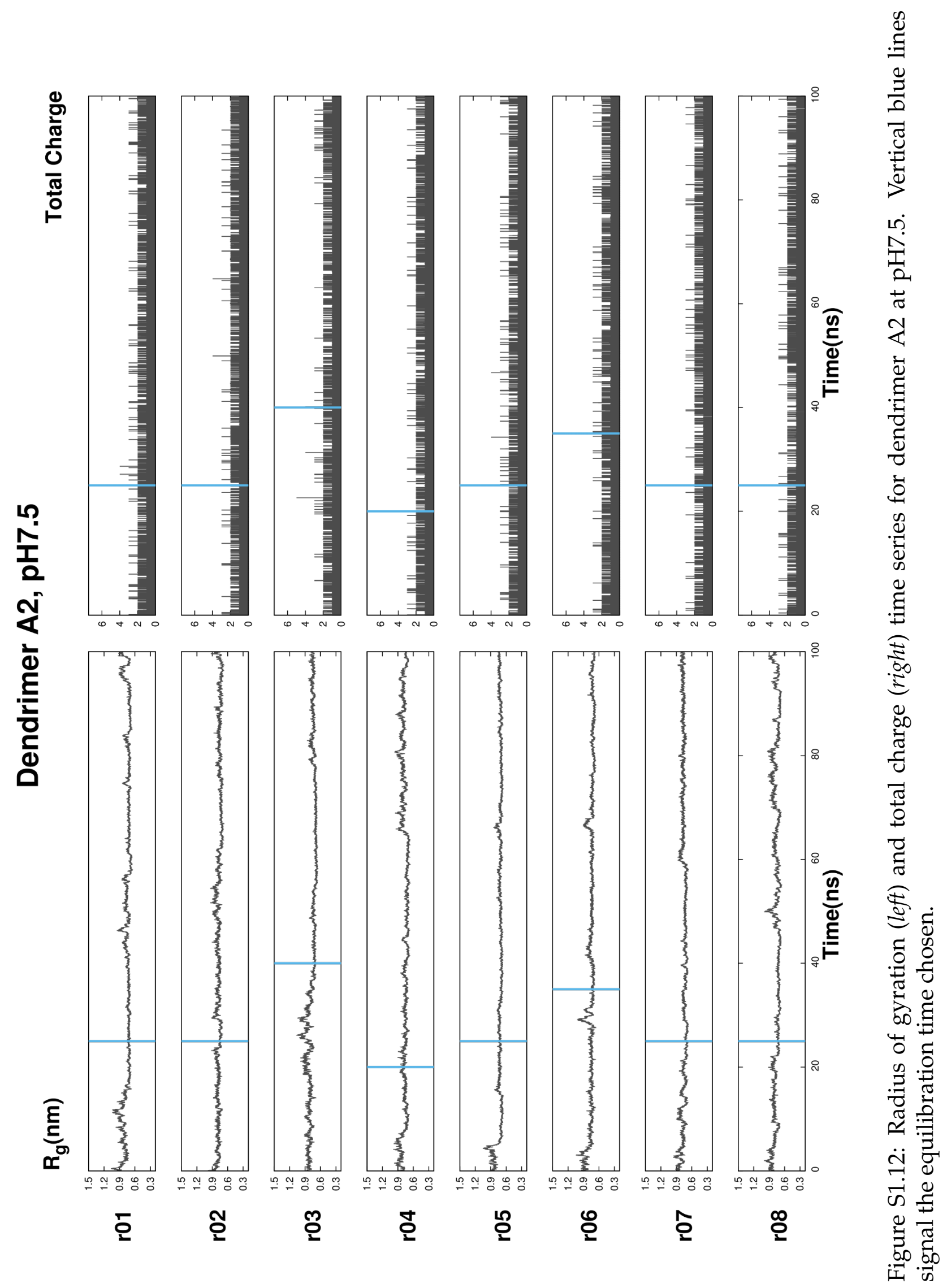




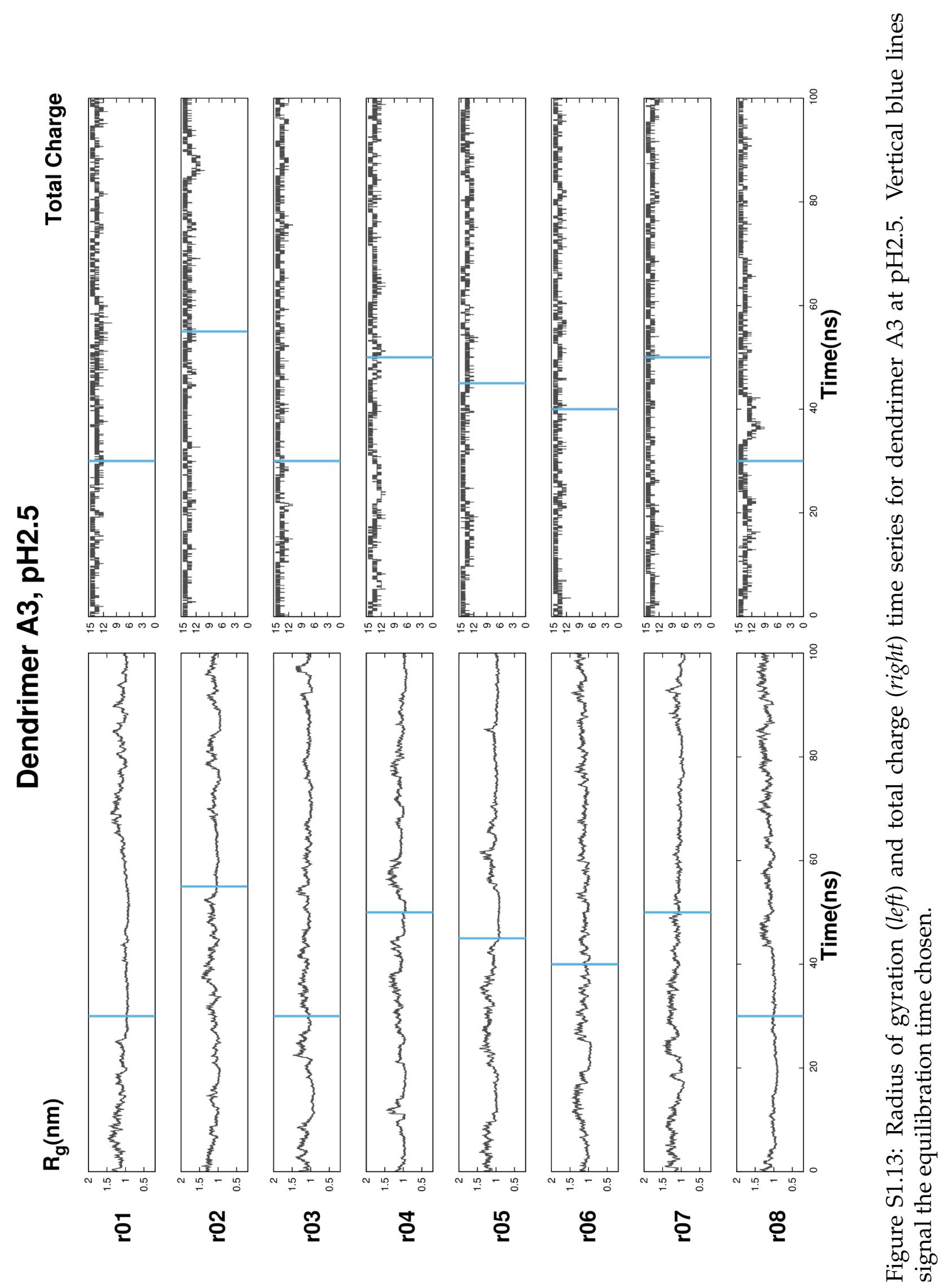




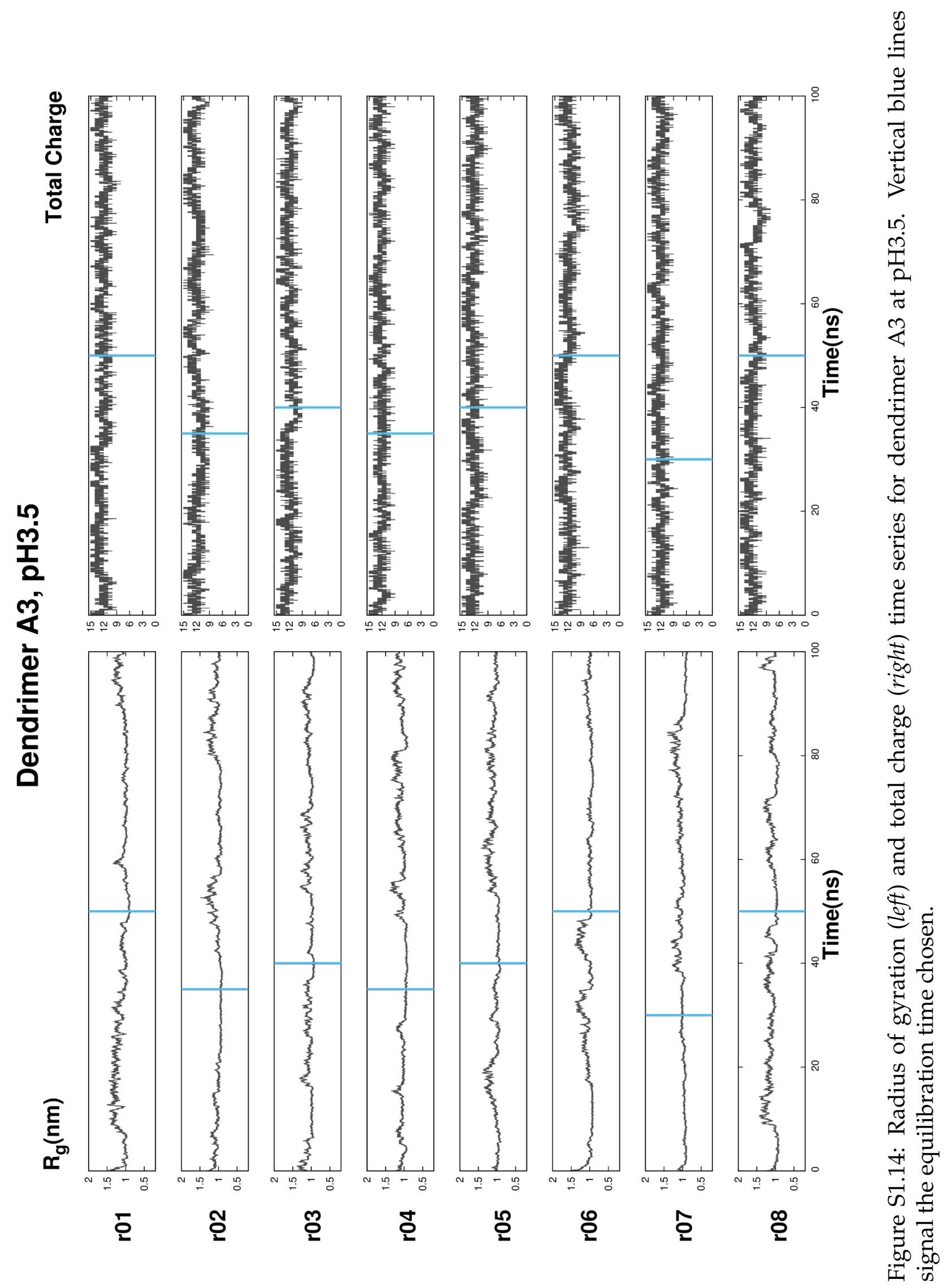




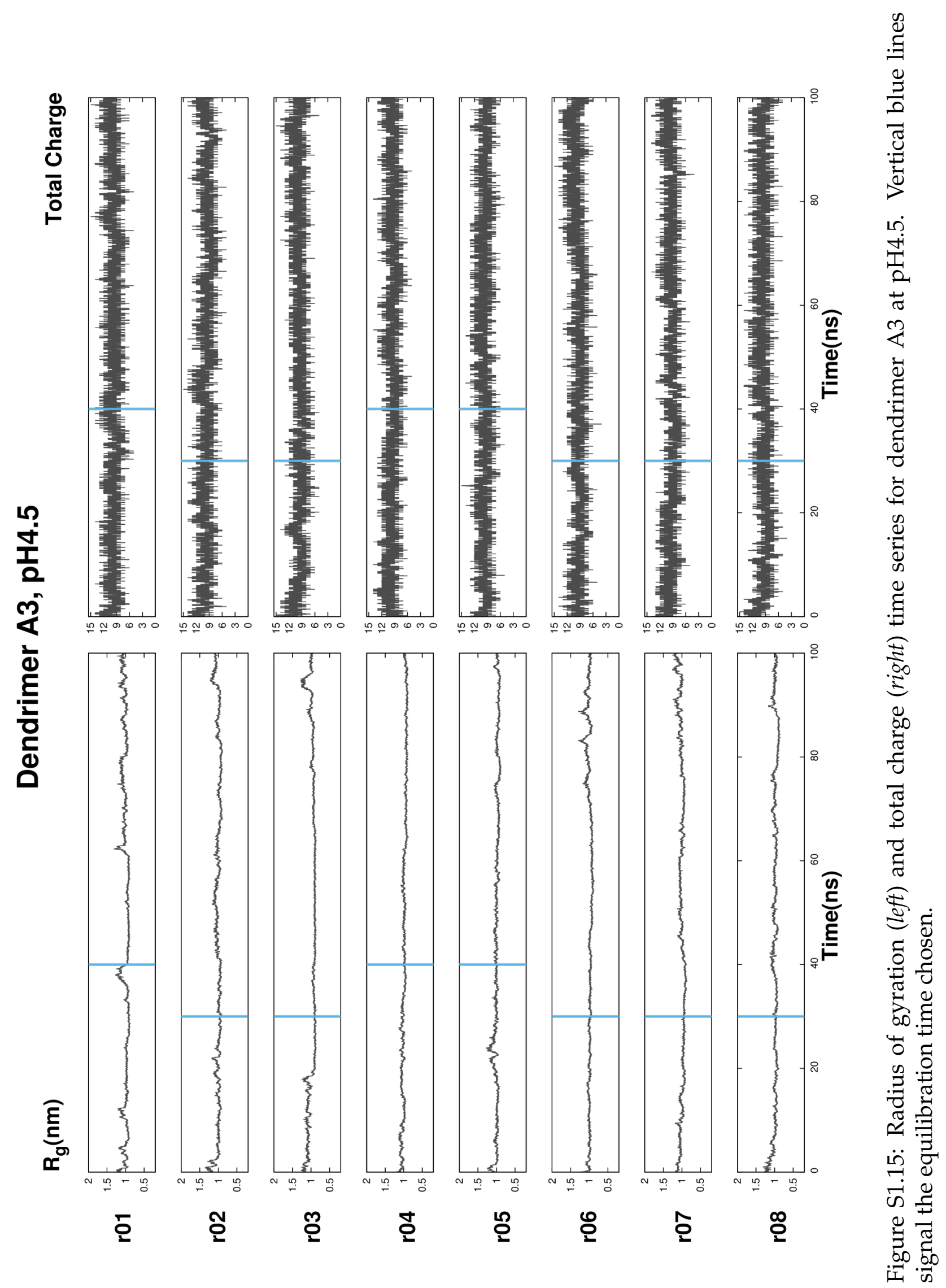




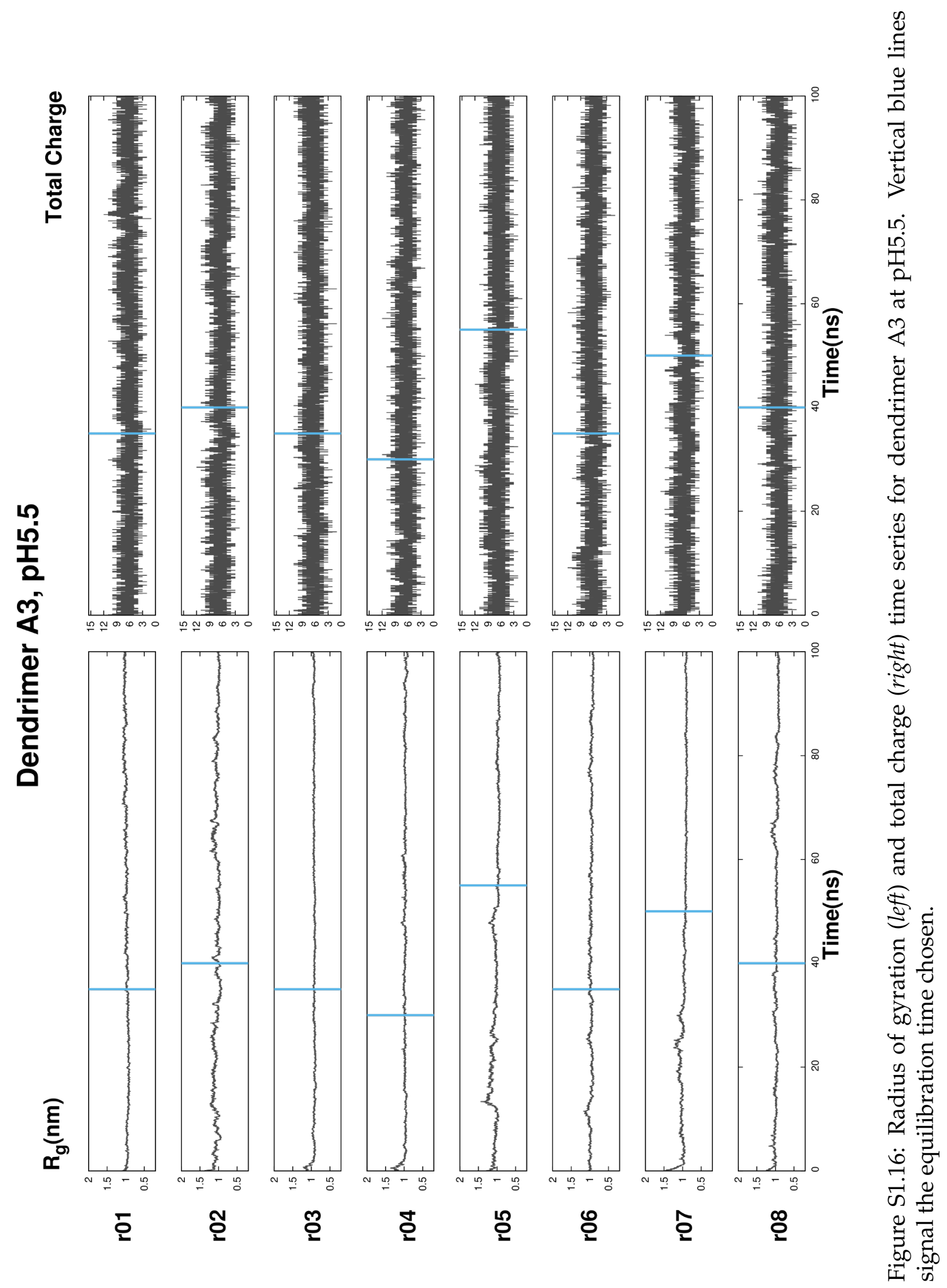




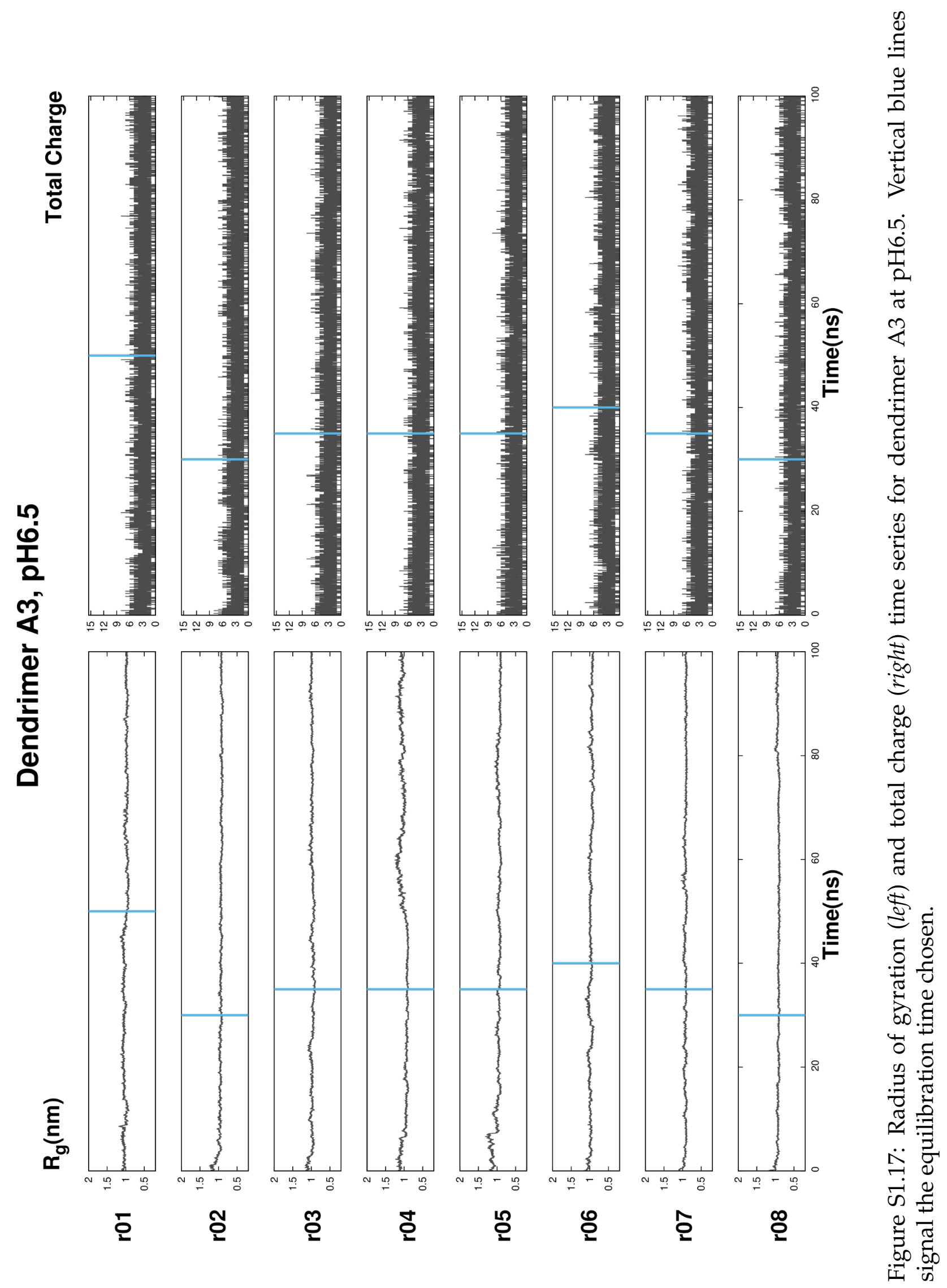




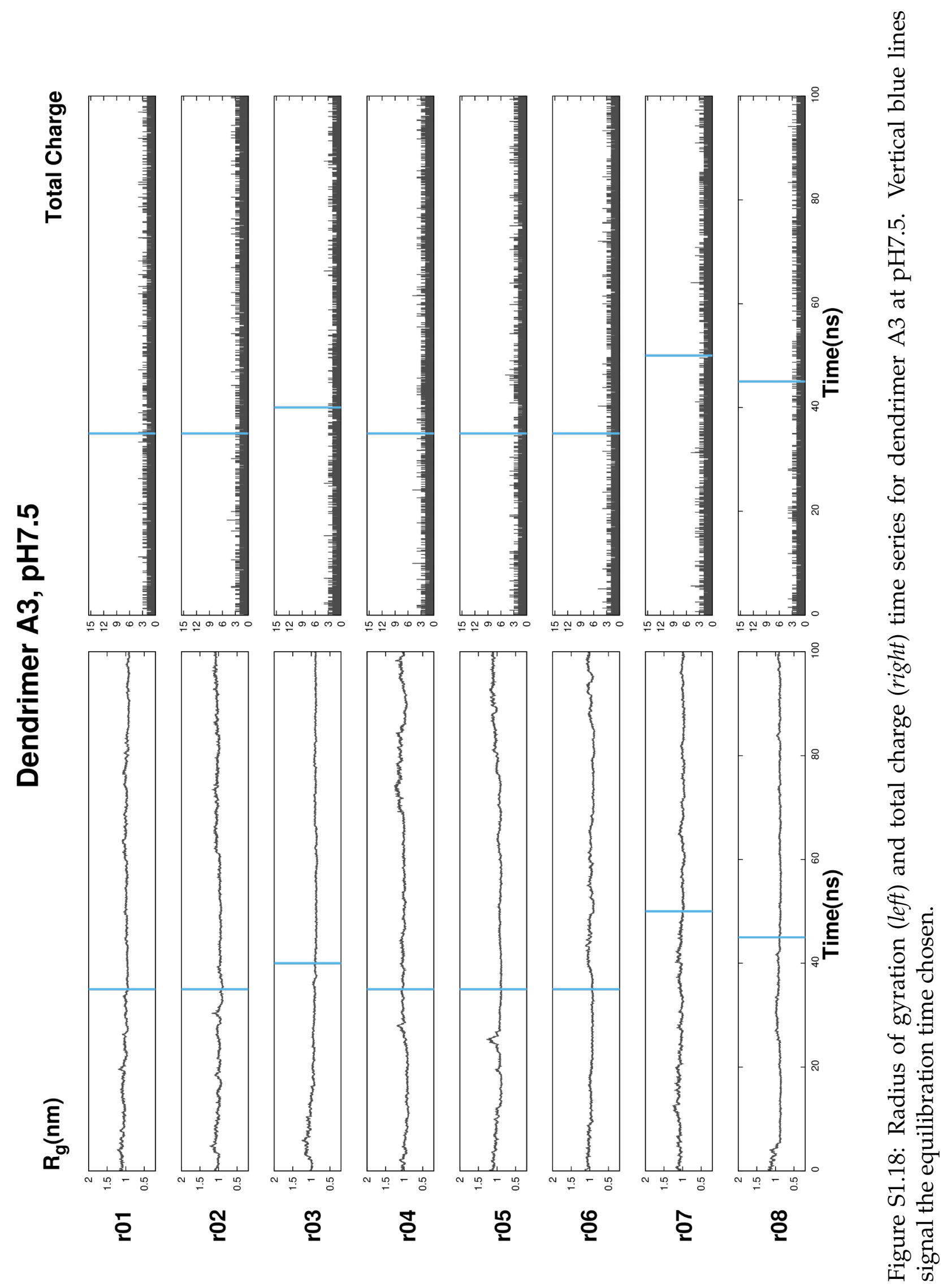




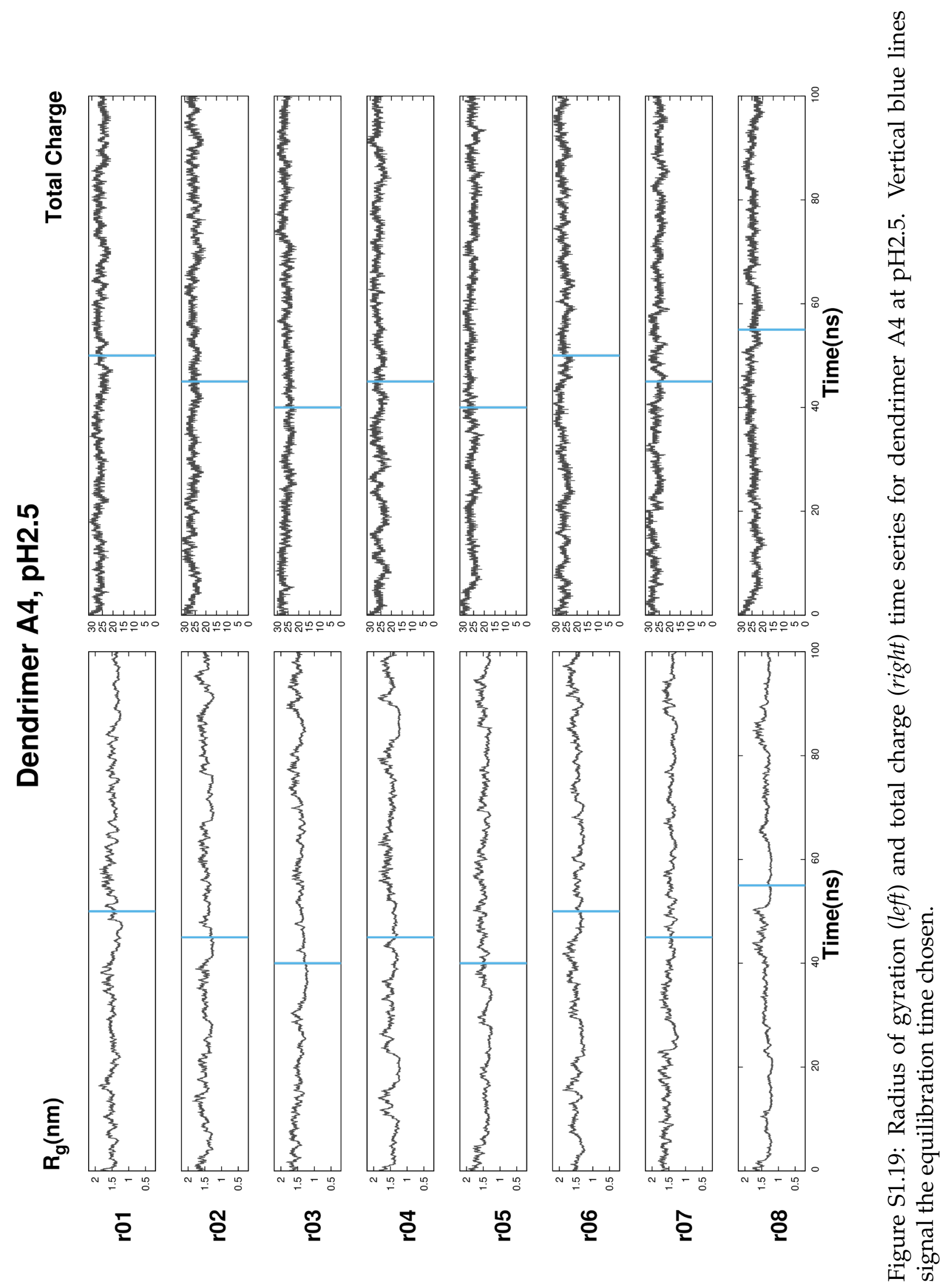




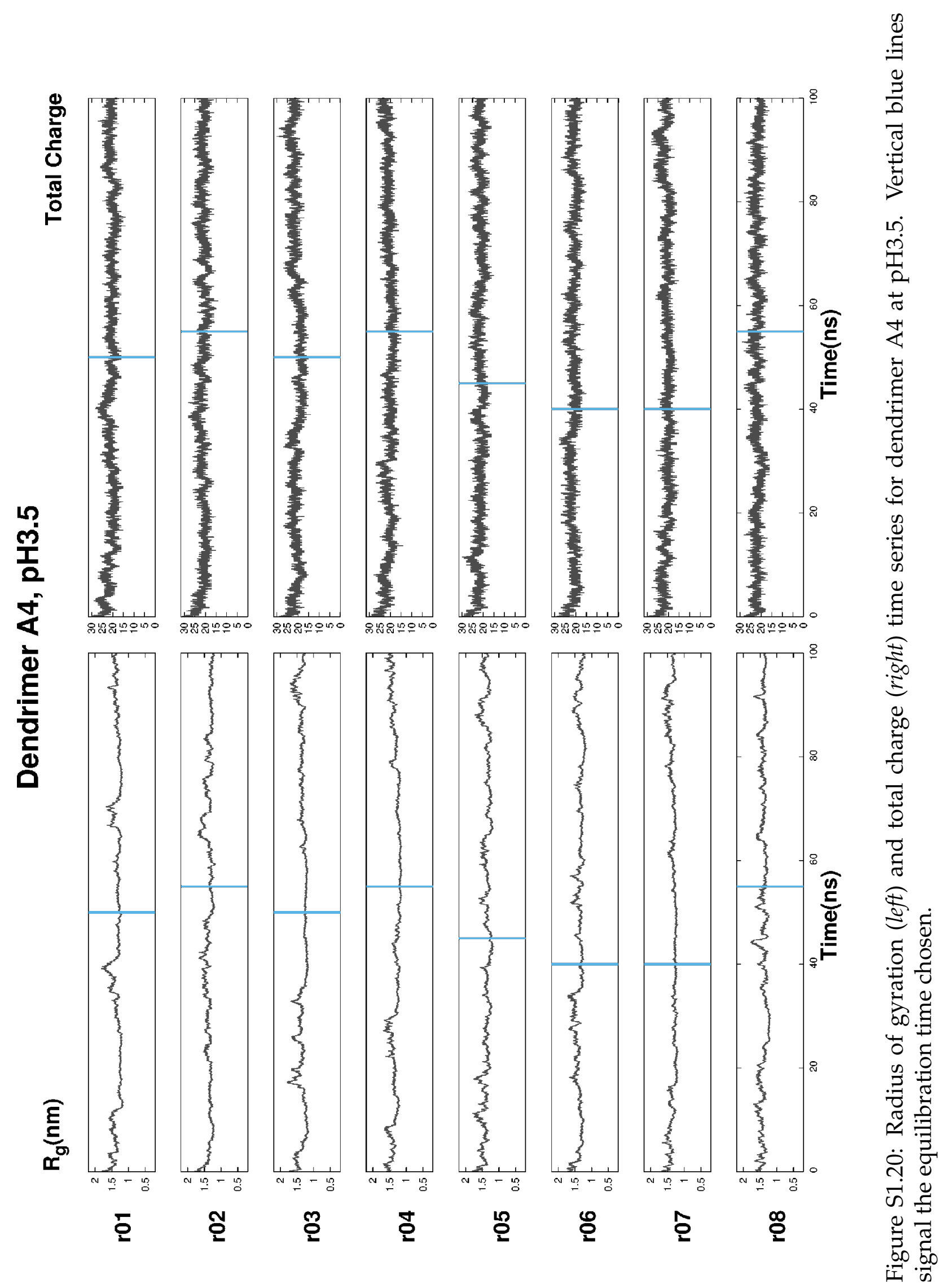




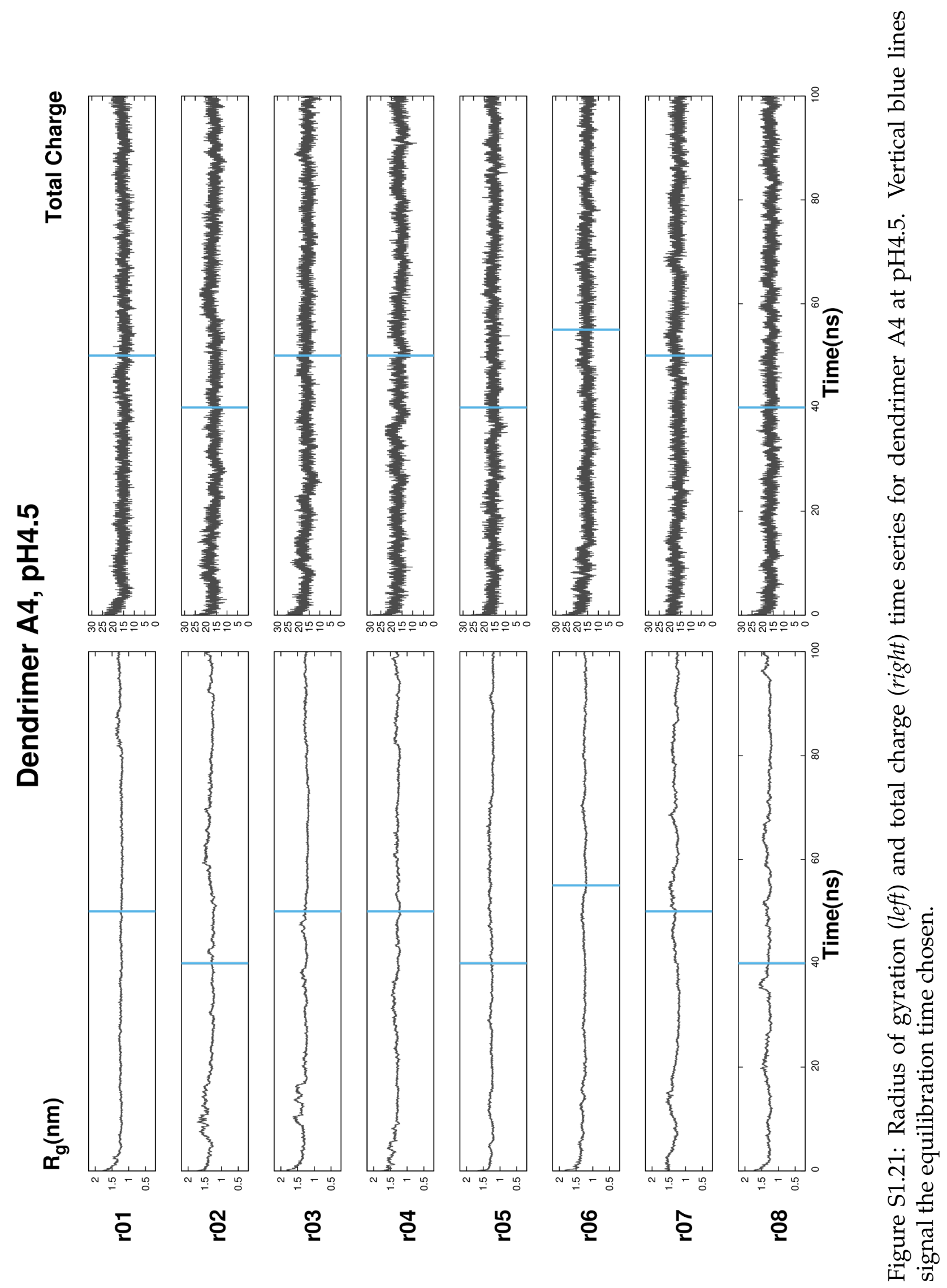




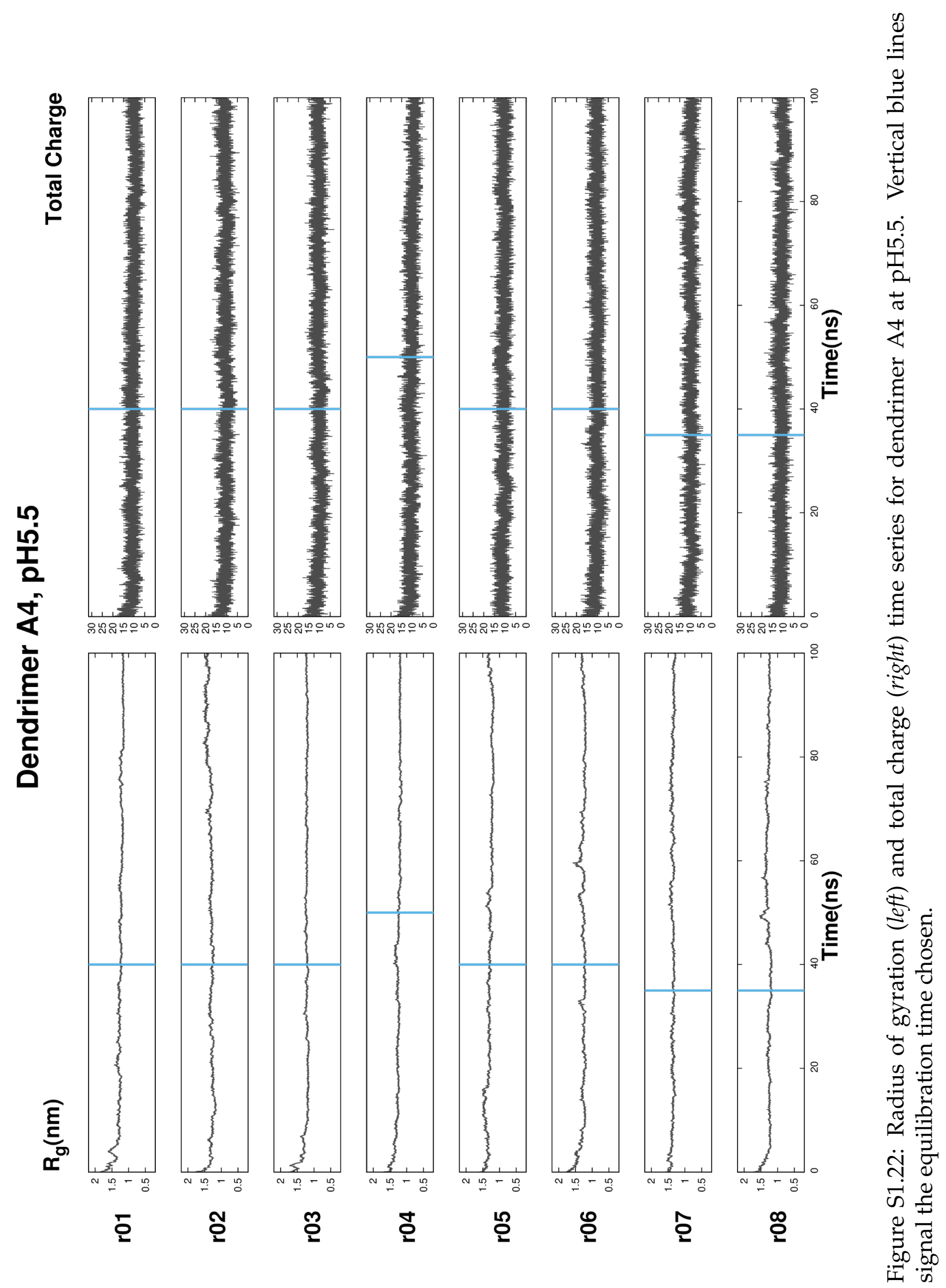




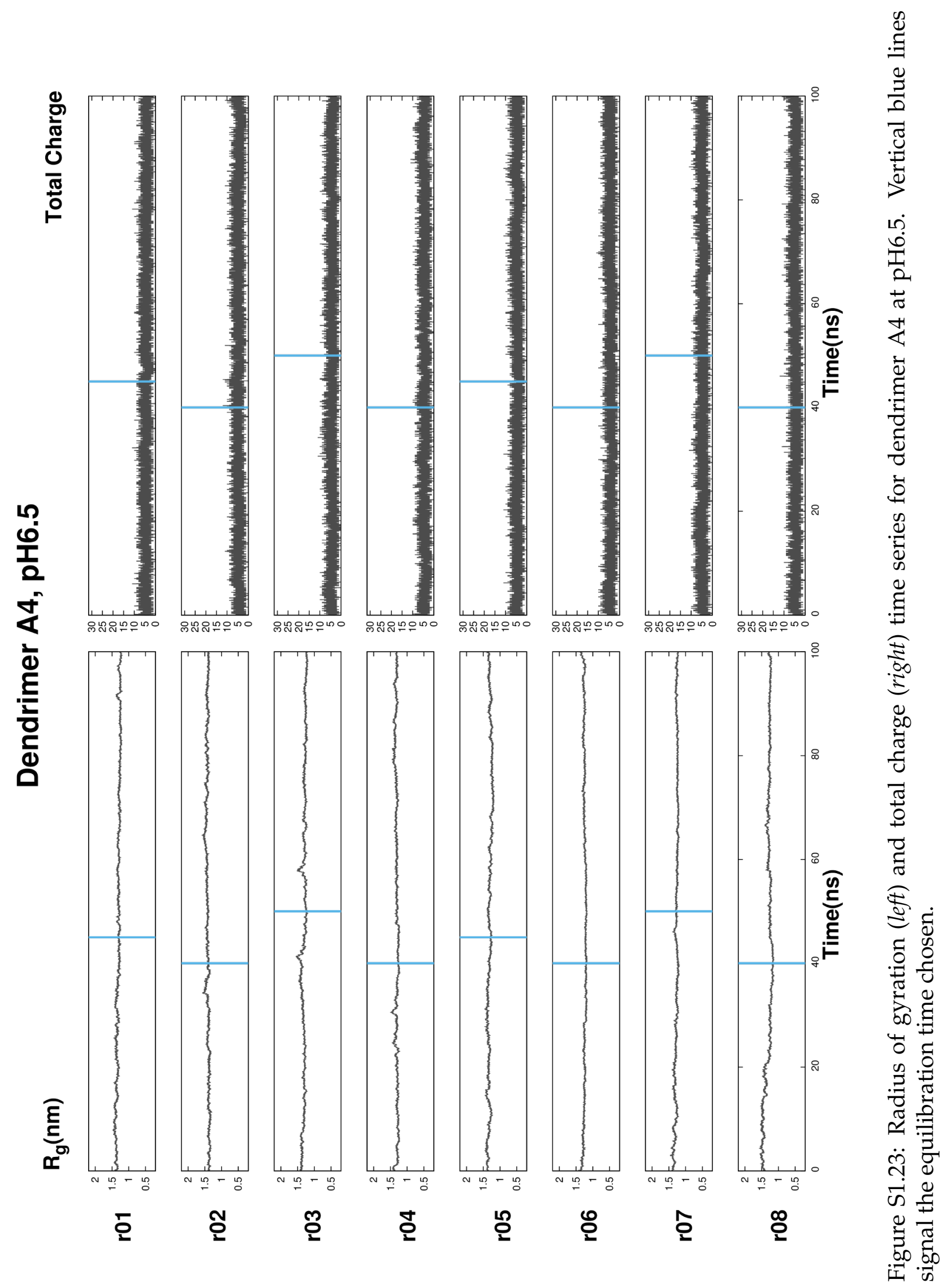




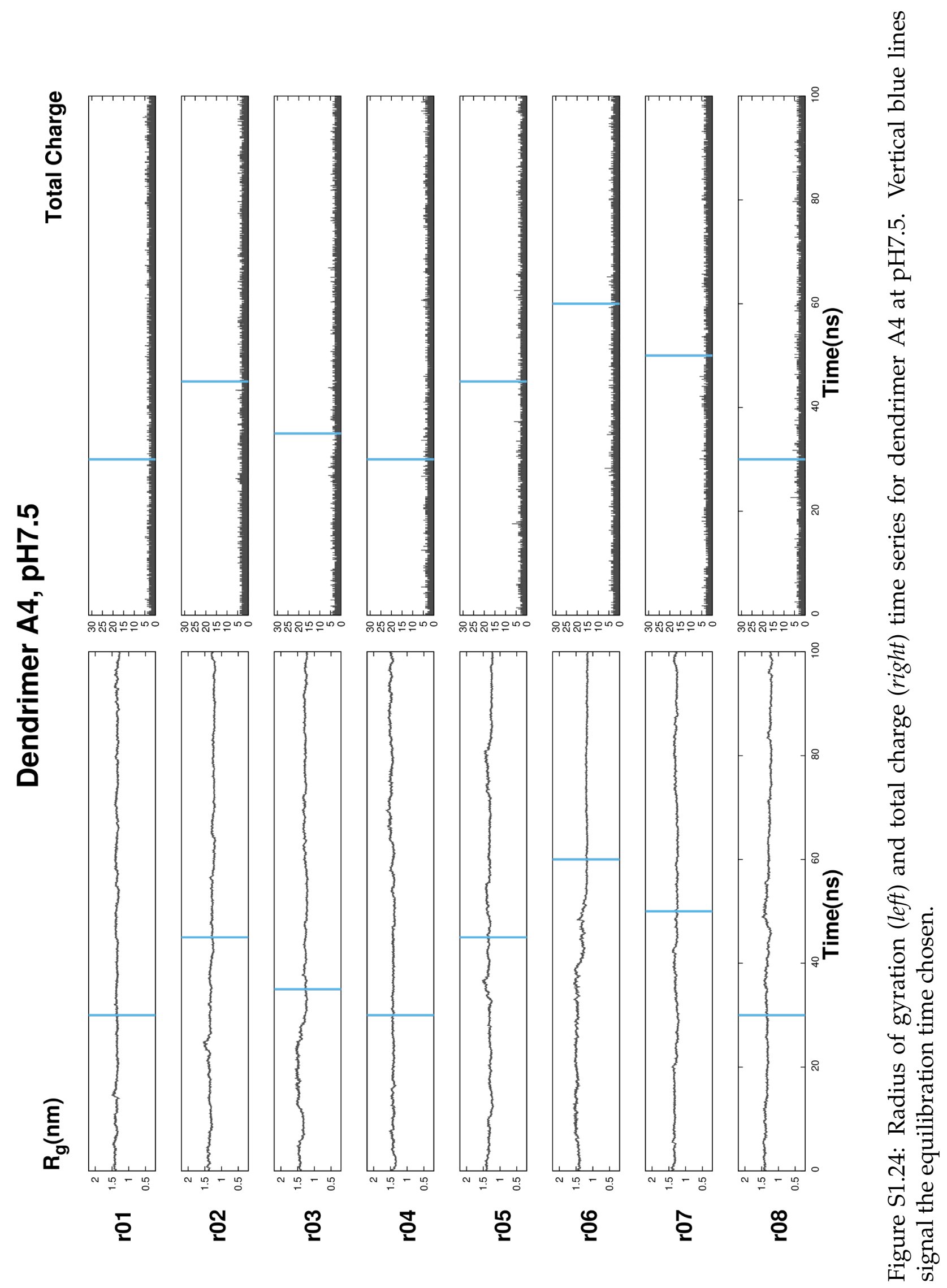


2 Replicate sampling convergence 
Dendrimer A1
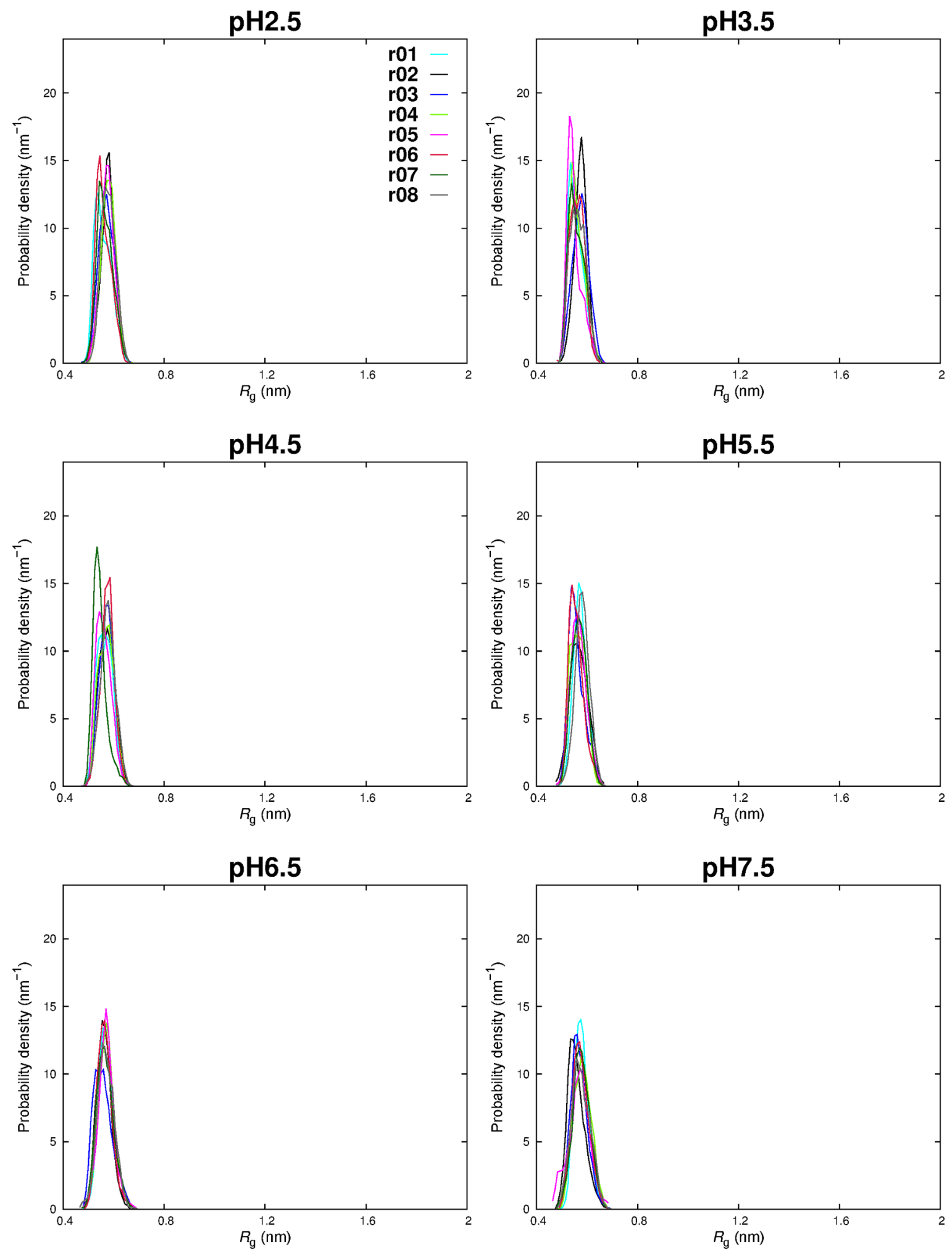

Figure S2.1: Dendrimer A1. Radius of gyration probability density histograms for the different replicates at the $\mathrm{pH}$ values studies. 
Dendrimer A2
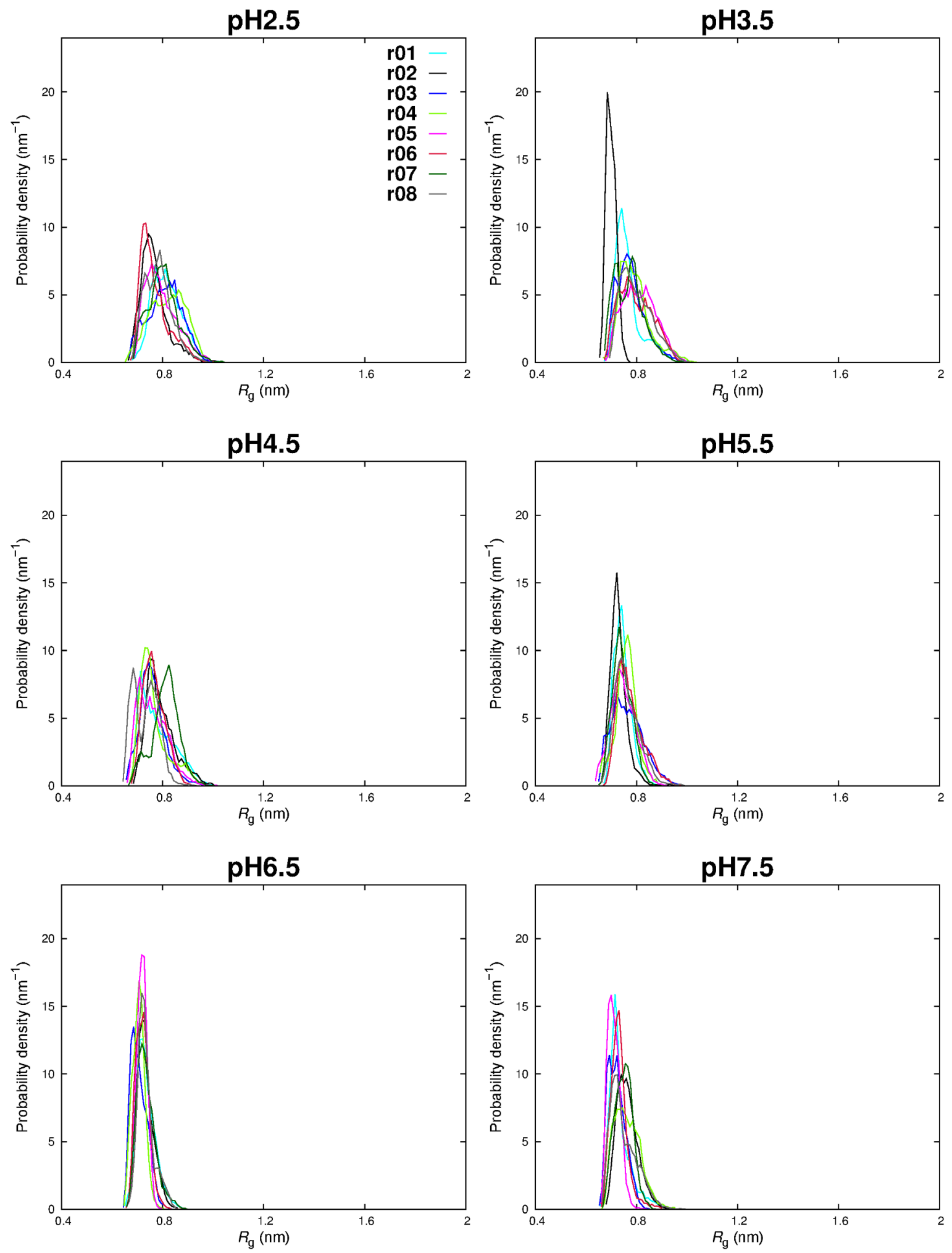

Figure S2.2: Dendrimer A2. Radius of gyration probability density histograms for the different replicates at the $\mathrm{pH}$ values studies. 


\section{Dendrimer A3}
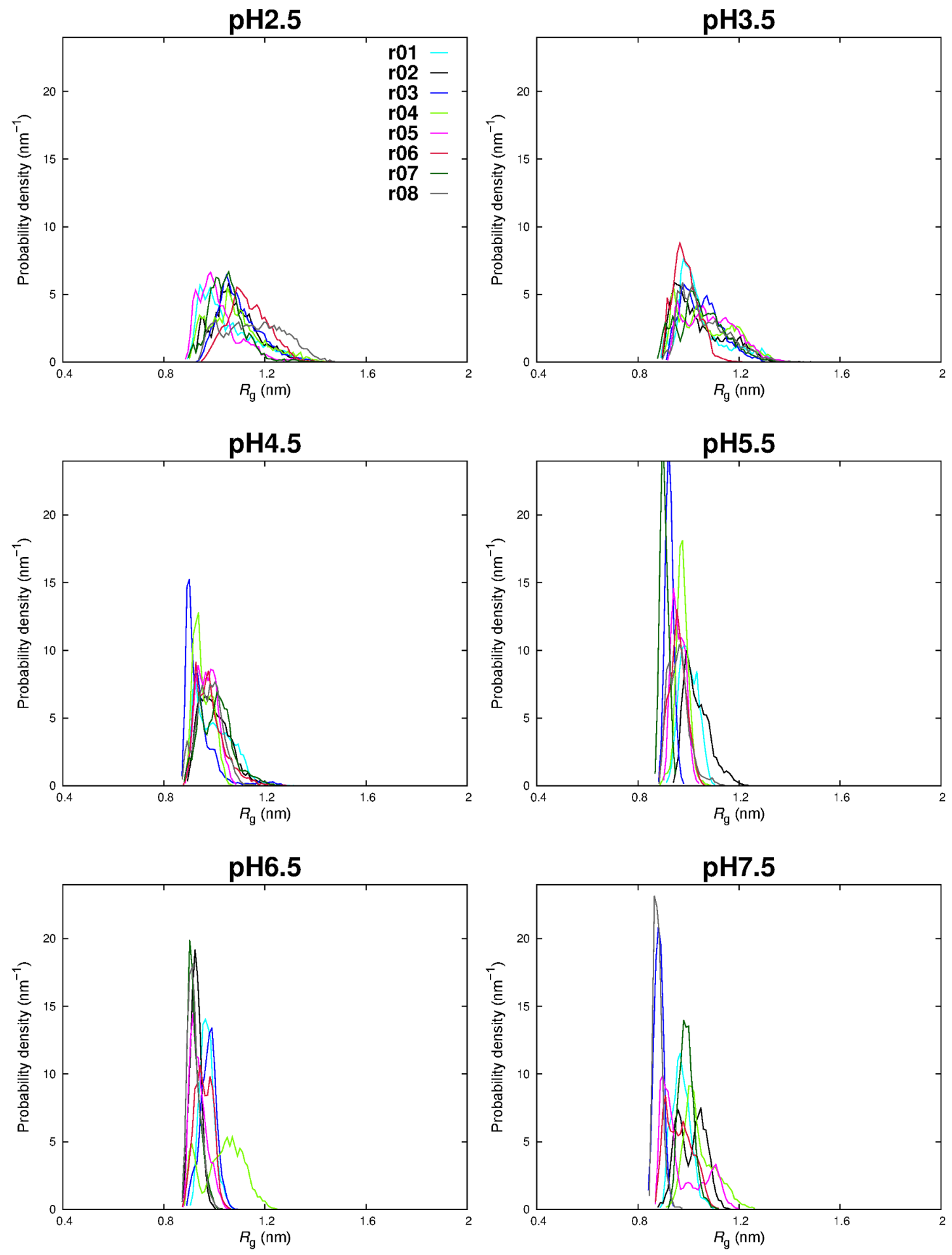

Figure S2.3: Dendrimer A3. Radius of gyration probability density histograms for the different replicates at the $\mathrm{pH}$ values studies. 
Dendrimer A4
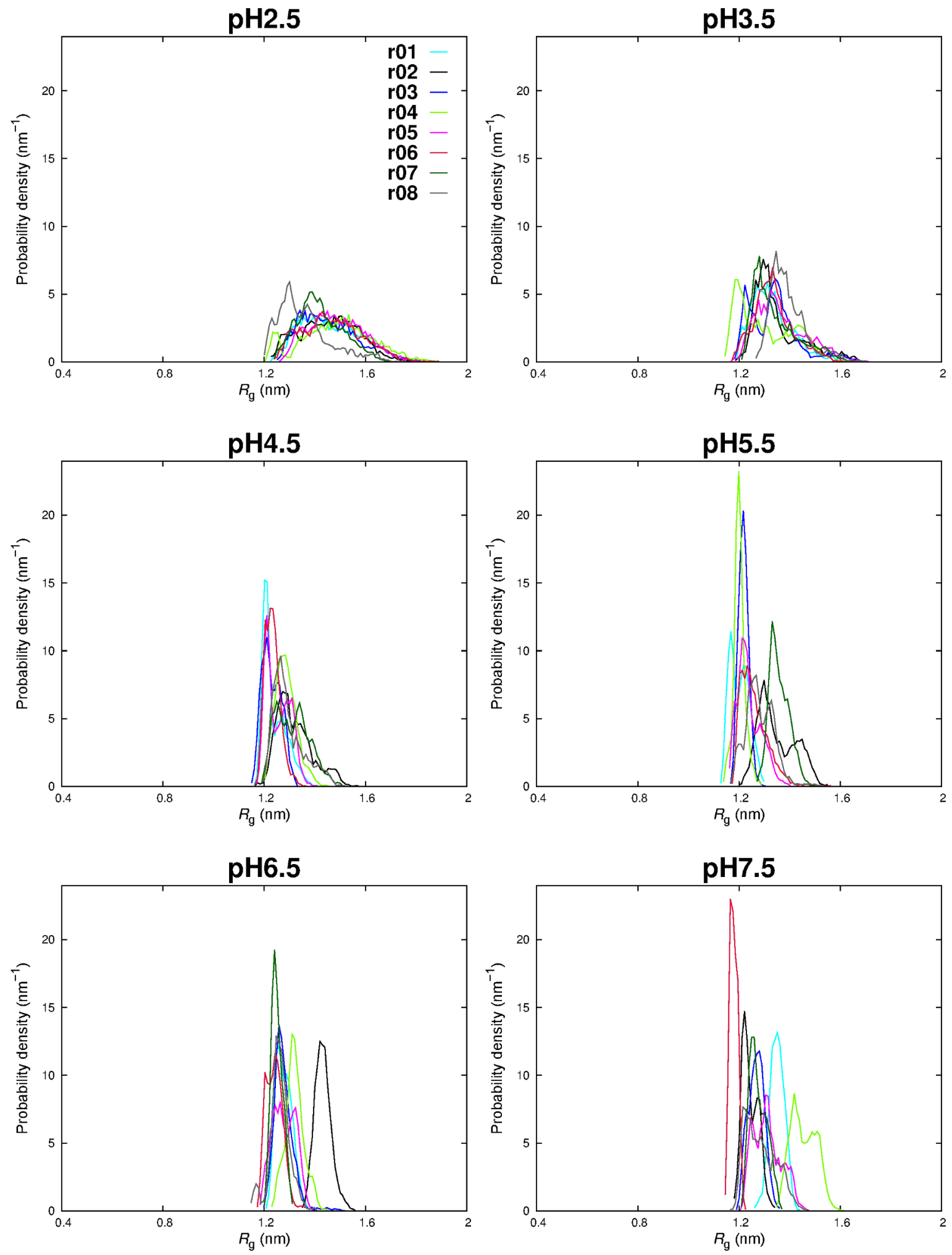

Figure S2.4: Dendrimer A4. Radius of gyration probability density histograms for the different replicates at the $\mathrm{pH}$ values studies. 


\section{Parameterization of substrate BPTS}

Partial atomic charges were determined using a procedure described elsewhere. ${ }^{1}$ The molecule geometry was optimized with Gaussian 03 at the HF/6-31G(d,p) level, and the resulting electrostatic potential was fitted to atomic coordinates with RESP. ${ }^{2}$ The final atomic charges were slightly adjusted to be consistent with the charge groups presented in Figure S3.1.

The bonded and van der Waals parameters for 8-butyryloxypyrene-1,3,6-trisulfonate were derived from the GROMOS 54A7 forcefield. ${ }^{3}$ The parameter macros presented in tables S3.1 to S3.6 must be interpreted having in mind the GROMACS package configuration. The abbreviations gb $x$, ga $x$, gd $x$ and gi $x$, mean gromos bond (gb), angle (ga), dihedral (gd) and improper (gi); the $\mathrm{x}$ accounts for a number that is associated with a certain parameter value in the force field. 


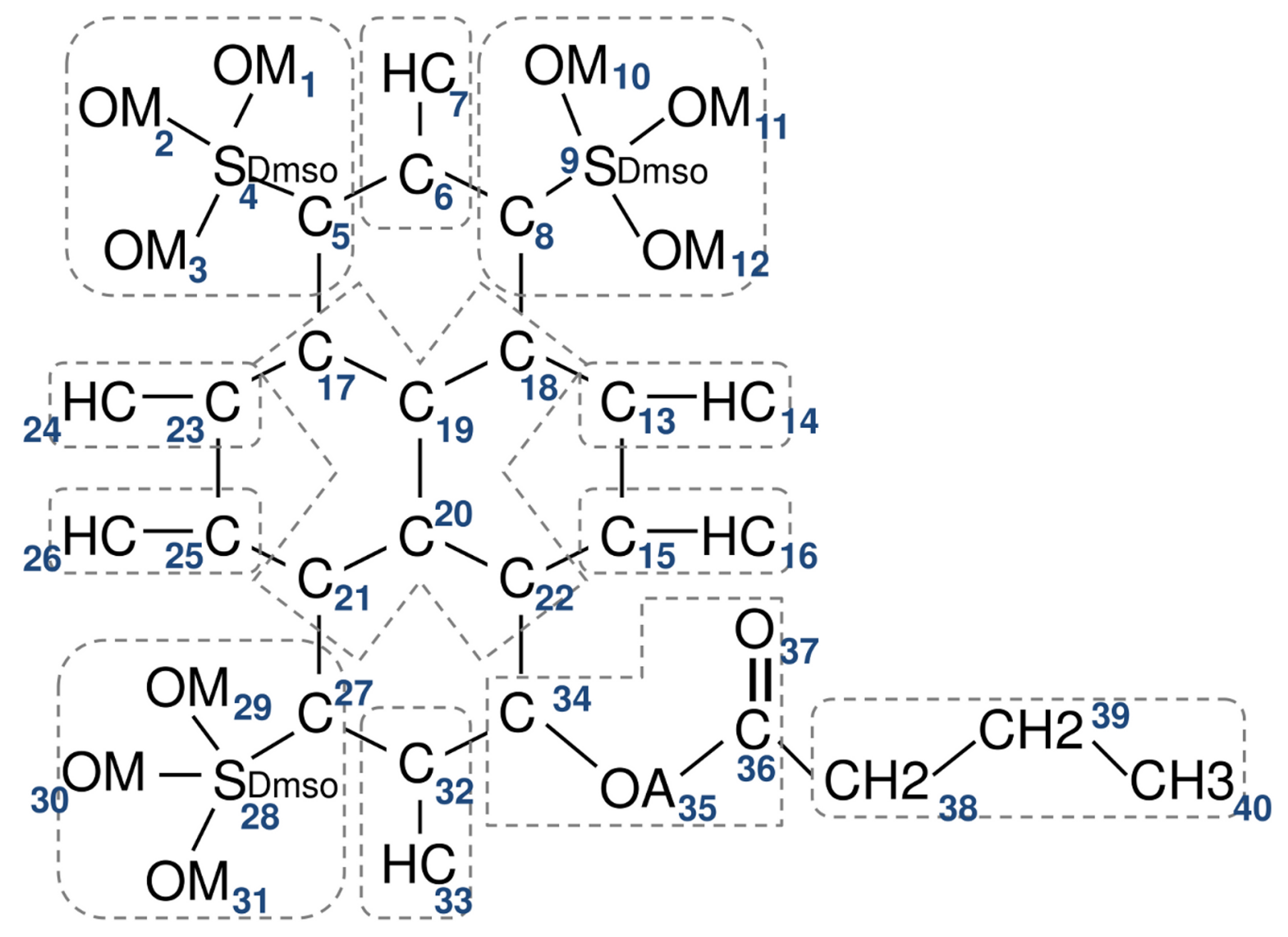

Figure S3.1: Atom numbering and atom types for 8-butyryloxypyrene-1,3,6-trisulfonate (substrate BPTS). Dashed lines delimit the charged-groups considered. 
Table S3.1: Atomic partial charges of BPTS

\begin{tabular}{rrrrrrrr}
\hline Atom & Charge & & Atom & Charge & & Atom & Charge \\
\cline { 1 - 2 } \cline { 7 - 8 } $\mathbf{1}$ & -0.610 & & $\mathbf{1 5}$ & -0.140 & & $\mathbf{2 9}$ & -0.610 \\
$\mathbf{2}$ & -0.610 & & $\mathbf{1 6}$ & 0.140 & & $\mathbf{3 0}$ & -0.610 \\
$\mathbf{3}$ & -0.610 & & $\mathbf{1 7}$ & 0.000 & & $\mathbf{3 1}$ & -0.610 \\
$\mathbf{4}$ & 0.910 & & $\mathbf{1 8}$ & 0.000 & & $\mathbf{3 2}$ & -0.140 \\
$\mathbf{5}$ & -0.080 & & $\mathbf{1 9}$ & 0.000 & & $\mathbf{3 3}$ & 0.140 \\
$\mathbf{6}$ & -0.140 & & $\mathbf{2 0}$ & 0.000 & & $\mathbf{3 4}$ & 0.340 \\
$\mathbf{7}$ & 0.140 & & $\mathbf{2 1}$ & 0.000 & & $\mathbf{3 5}$ & -0.400 \\
$\mathbf{8}$ & -0.080 & & $\mathbf{2 2}$ & 0.000 & & $\mathbf{3 6}$ & 0.580 \\
$\mathbf{9}$ & 0.910 & & $\mathbf{2 3}$ & -0.140 & & $\mathbf{3 7}$ & -0.520 \\
$\mathbf{1 0}$ & -0.610 & & $\mathbf{2 4}$ & 0.140 & & $\mathbf{3 8}$ & 0.000 \\
$\mathbf{1 1}$ & -0.610 & & $\mathbf{2 5}$ & -0.140 & & $\mathbf{3 9}$ & 0.000 \\
$\mathbf{1 2}$ & -0.610 & & $\mathbf{2 6}$ & 0.140 & & $\mathbf{4 0}$ & 0.000 \\
$\mathbf{1 3}$ & -0.140 & & $\mathbf{2 7}$ & -0.080 & & & \\
$\mathbf{1 4}$ & 0.140 & $\mathbf{2 8}$ & 0.910 & & \\
\hline
\end{tabular}


Table S3.2: Bond types of BPTS

\begin{tabular}{|c|c|c|c|c|c|}
\hline Atom(i) & Atom(j) & Bond type & Atom(i) & Atom(j) & Bond type \\
\hline 1 & 4 & $\mathrm{gb} 25$ & 20 & 22 & gb 16 \\
\hline 2 & 4 & $\mathrm{gb} 25$ & 21 & 20 & gb 16 \\
\hline 3 & 4 & $\mathrm{gb} 25$ & 21 & 27 & gb 16 \\
\hline 4 & 5 & $\mathrm{gb} 32$ & 22 & 34 & gb 16 \\
\hline 5 & 17 & $\mathrm{gb} 16$ & 23 & 24 & gb 3 \\
\hline 5 & 6 & $\mathrm{gb} 16$ & 23 & 25 & gb 16 \\
\hline 6 & 7 & $\mathrm{gb} 3$ & 25 & 26 & gb 3 \\
\hline 6 & 8 & gb 16 & 25 & 21 & gb 16 \\
\hline 8 & 9 & gb 32 & 27 & 32 & gb 16 \\
\hline 8 & 18 & gb 16 & 27 & 28 & gb 32 \\
\hline 9 & 10 & gb 25 & 28 & 29 & gb 25 \\
\hline 9 & 11 & gb 25 & 28 & 30 & gb 25 \\
\hline 9 & 12 & gb 25 & 28 & 31 & gb 25 \\
\hline 13 & 14 & $\mathrm{gb} 3$ & 32 & 33 & $\mathrm{gb} 3$ \\
\hline 13 & 15 & $\mathrm{gb} 16$ & 32 & 34 & gb 16 \\
\hline 15 & 16 & gb 3 & 34 & 35 & gb 18 \\
\hline 15 & 22 & $\mathrm{gb} 16$ & 35 & 36 & gb 13 \\
\hline 17 & 23 & gb 16 & 36 & 37 & gb 5 \\
\hline 18 & 13 & $\mathrm{gb} 16$ & 36 & 38 & gb 27 \\
\hline 18 & 19 & gb 16 & 38 & 39 & gb 27 \\
\hline 19 & 20 & $\mathrm{gb} 16$ & 39 & 40 & gb 27 \\
\hline 19 & 17 & $\mathrm{gb} 16$ & & & \\
\hline
\end{tabular}


Table S3.3: Angle types of BPTS

\begin{tabular}{|c|c|c|c|c|c|c|c|}
\hline Atom(i) & Atom(j) & Atom(k) & Angle type & Atom(i) & Atom(j) & Atom(k) & Angle type \\
\hline 1 & 4 & 2 & ga 14 & 17 & 23 & 24 & ga 25 \\
\hline 1 & 4 & 3 & ga 14 & 17 & 23 & 25 & ga 27 \\
\hline 1 & 4 & 5 & ga 46 & 18 & 19 & 20 & ga 27 \\
\hline 2 & 4 & 3 & ga 29 & 19 & 17 & 23 & ga 27 \\
\hline 2 & 4 & 5 & ga 46 & 19 & 20 & 21 & ga 27 \\
\hline 3 & 4 & 5 & ga 46 & 19 & 20 & 22 & ga 27 \\
\hline 4 & 5 & 6 & ga 35 & 20 & 21 & 25 & ga 27 \\
\hline 4 & 5 & 17 & ga 35 & 20 & 21 & 27 & ga 27 \\
\hline 5 & 6 & 7 & ga 25 & 20 & 22 & 34 & ga 27 \\
\hline 5 & 6 & 8 & ga 27 & 21 & 20 & 22 & ga 27 \\
\hline 5 & 17 & 19 & ga 27 & 21 & 25 & 23 & ga 27 \\
\hline 5 & 17 & 23 & ga 35 & 21 & 25 & 26 & ga 25 \\
\hline 6 & 5 & 17 & ga 27 & 21 & 27 & 28 & ga 35 \\
\hline 6 & 8 & 9 & ga 35 & 21 & 27 & 32 & ga 27 \\
\hline 6 & 8 & 18 & ga 27 & 22 & 34 & 32 & ga 27 \\
\hline 7 & 6 & 8 & ga 25 & 22 & 34 & 35 & ga 19 \\
\hline 8 & 9 & 10 & ga 46 & 23 & 25 & 26 & ga 25 \\
\hline 8 & 9 & 11 & ga 46 & 24 & 23 & 25 & ga 25 \\
\hline 8 & 9 & 12 & ga 46 & 25 & 21 & 27 & ga 35 \\
\hline 8 & 18 & 13 & ga 35 & 27 & 28 & 29 & ga 46 \\
\hline 8 & 18 & 19 & ga 27 & 27 & 28 & 30 & ga 46 \\
\hline 9 & 8 & 18 & ga 35 & 27 & 28 & 31 & ga 46 \\
\hline 10 & 9 & 11 & ga 14 & 27 & 32 & 33 & ga 25 \\
\hline 10 & 9 & 12 & ga 14 & 27 & 32 & 34 & ga 27 \\
\hline 11 & 9 & 12 & ga 29 & 28 & 27 & 32 & ga 35 \\
\hline 13 & 15 & 16 & ga 25 & 29 & 28 & 30 & ga 14 \\
\hline 13 & 15 & 22 & ga 27 & 29 & 28 & 31 & ga 14 \\
\hline 13 & 18 & 19 & ga 27 & 30 & 28 & 31 & ga 29 \\
\hline 14 & 13 & 15 & ga 25 & 32 & 34 & 35 & ga 19 \\
\hline 14 & 13 & 18 & ga 25 & 33 & 32 & 34 & ga 25 \\
\hline 15 & 13 & 18 & ga 27 & 34 & 35 & 36 & ga 12 \\
\hline 15 & 22 & 20 & ga 27 & 35 & 36 & 37 & ga 33 \\
\hline 15 & 22 & 34 & ga 35 & 35 & 36 & 38 & ga 19 \\
\hline 16 & 15 & 22 & ga 25 & 36 & 38 & 39 & ga 15 \\
\hline 17 & 18 & 19 & ga 27 & 37 & 36 & 38 & ga 30 \\
\hline 17 & 19 & 20 & ga 27 & 38 & 39 & 40 & ga 15 \\
\hline
\end{tabular}


Table S3.4: Dihedrals of BPTS

\begin{tabular}{|c|c|c|c|c|c|c|c|c|c|c|c|}
\hline$i$ & $j$ & $k$ & $n$ & type & dihedral & $i$ & $j$ & $k$ & $n$ & type & dihedral \\
\hline 6 & 5 & 4 & 3 & 1 & $\operatorname{gd} 40$ & 19 & 20 & 22 & 15 & 2 & gi 1 \\
\hline 6 & 8 & 9 & 12 & 1 & $\operatorname{gd} 40$ & 20 & 19 & 18 & 13 & 2 & gi 1 \\
\hline 22 & 34 & 35 & 36 & 1 & $\operatorname{gd} 17$ & 20 & 21 & 19 & 22 & 2 & gi 1 \\
\hline 32 & 27 & 28 & 29 & 1 & $\operatorname{gd~} 40$ & 20 & 22 & 15 & 13 & 2 & gi 1 \\
\hline 34 & 35 & 36 & 38 & 1 & $\operatorname{gd} 11$ & 21 & 20 & 27 & 25 & 2 & gi 1 \\
\hline 35 & 36 & 38 & 39 & 1 & $\operatorname{gd} 40$ & 22 & 15 & 13 & 18 & 2 & gi 1 \\
\hline 36 & 38 & 39 & 40 & 1 & $\operatorname{gd} 34$ & 22 & 15 & 34 & 20 & 2 & gi 1 \\
\hline 5 & 6 & 4 & 17 & 2 & gi 1 & 22 & 20 & 19 & 18 & 2 & gi 1 \\
\hline 5 & 6 & 8 & 18 & 2 & gi 1 & 23 & 17 & 19 & 20 & 2 & gi 1 \\
\hline 5 & 17 & 19 & 18 & 2 & gi 1 & 23 & 17 & 24 & 25 & 2 & gi 1 \\
\hline 8 & 6 & 9 & 18 & 2 & gi 1 & 23 & 25 & 21 & 20 & 2 & gi 1 \\
\hline 13 & 18 & 14 & 15 & 2 & gi 1 & 25 & 21 & 20 & 19 & 2 & gi 1 \\
\hline 15 & 13 & 16 & 22 & 2 & gi 1 & 25 & 21 & 26 & 23 & 2 & gi 1 \\
\hline 17 & 5 & 6 & 8 & 2 & gi 1 & 25 & 23 & 17 & 19 & 2 & gi 1 \\
\hline 17 & 19 & 5 & 23 & 2 & gi 1 & 27 & 21 & 20 & 22 & 2 & gi 1 \\
\hline 17 & 19 & 18 & 8 & 2 & gi 1 & 27 & 21 & 28 & 32 & 2 & gi 1 \\
\hline 17 & 19 & 20 & 21 & 2 & gi 1 & 27 & 32 & 34 & 22 & 2 & gi 1 \\
\hline 17 & 23 & 25 & 21 & 2 & gi 1 & 32 & 27 & 21 & 20 & 2 & gi 1 \\
\hline 18 & 13 & 8 & 19 & 2 & gi 1 & 32 & 34 & 22 & 20 & 2 & gi 1 \\
\hline 19 & 17 & 5 & 6 & 2 & gi 1 & 34 & 22 & 20 & 21 & 2 & gi 1 \\
\hline 19 & 17 & 20 & 18 & 2 & gi 1 & 34 & 22 & 35 & 32 & 2 & gi 1 \\
\hline 19 & 18 & 8 & 6 & 2 & gi 1 & 34 & 32 & 27 & 21 & 2 & gi 1 \\
\hline 19 & 18 & 13 & 15 & 2 & gi 1 & 36 & 35 & 37 & 38 & 2 & gi 1 \\
\hline
\end{tabular}


Table S3.5: Pairs in BPTS

\begin{tabular}{ccccccc}
\hline Atom(i) & Atom(j) & type & & Atom(i) & Atom(j) & type \\
\cline { 1 - 3 } \cline { 5 - 6 } $\mathbf{1}$ & $\mathbf{6}$ & 1 & & $\mathbf{2 1}$ & $\mathbf{2 9}$ & 1 \\
$\mathbf{1}$ & $\mathbf{1 7}$ & 1 & & $\mathbf{2 1}$ & $\mathbf{3 0}$ & 1 \\
$\mathbf{2}$ & $\mathbf{6}$ & 1 & & $\mathbf{2 1}$ & $\mathbf{3 1}$ & 1 \\
$\mathbf{2}$ & $\mathbf{1 7}$ & 1 & & $\mathbf{2 2}$ & $\mathbf{3 6}$ & 1 \\
$\mathbf{3}$ & $\mathbf{6}$ & 1 & & $\mathbf{2 9}$ & $\mathbf{3 2}$ & 1 \\
$\mathbf{3}$ & $\mathbf{1 7}$ & 1 & & $\mathbf{3 0}$ & $\mathbf{3 2}$ & 1 \\
$\mathbf{6}$ & $\mathbf{1 0}$ & 1 & & $\mathbf{3 1}$ & $\mathbf{3 2}$ & 1 \\
$\mathbf{6}$ & $\mathbf{1 1}$ & 1 & & $\mathbf{3 2}$ & $\mathbf{3 6}$ & 1 \\
$\mathbf{6}$ & $\mathbf{1 2}$ & 1 & & $\mathbf{3 4}$ & $\mathbf{3 7}$ & 1 \\
$\mathbf{1 0}$ & $\mathbf{1 8}$ & 1 & & $\mathbf{3 4}$ & $\mathbf{3 8}$ & 1 \\
$\mathbf{1 1}$ & $\mathbf{1 8}$ & 1 & & $\mathbf{3 6}$ & $\mathbf{4 0}$ & 1 \\
$\mathbf{1 2}$ & $\mathbf{1 8}$ & 1 & & $\mathbf{3 7}$ & $\mathbf{3 9}$ & 1 \\
\hline
\end{tabular}


Table S3.6: Exclusions in BPTS

\begin{tabular}{|c|c|c|c|c|c|}
\hline Atom(i) & Atom(j) & Atom(i) & Atom(j) & Atom(i) & Atom $(j)$ \\
\hline 4 & 7 & 13 & 20 & 20 & 23 \\
\hline 4 & 8 & 13 & 34 & 20 & 26 \\
\hline 4 & 19 & 14 & 16 & 20 & 28 \\
\hline 4 & 23 & 14 & 19 & 20 & 32 \\
\hline 5 & 9 & 14 & 22 & 20 & 35 \\
\hline 5 & 18 & 15 & 19 & 21 & 24 \\
\hline 5 & 20 & 15 & 21 & 21 & 34 \\
\hline 5 & 24 & 15 & 32 & 22 & 25 \\
\hline 5 & 25 & 15 & 35 & 22 & 27 \\
\hline 6 & 13 & 16 & 18 & 22 & 33 \\
\hline 6 & 19 & 16 & 20 & 23 & 27 \\
\hline 6 & 23 & 16 & 34 & 24 & 26 \\
\hline 7 & 9 & 17 & 21 & 25 & 28 \\
\hline 7 & 17 & 17 & 22 & 25 & 32 \\
\hline 7 & 18 & 17 & 26 & 25 & 33 \\
\hline 8 & 14 & 18 & 21 & 26 & 27 \\
\hline 8 & 15 & 18 & 22 & 27 & 35 \\
\hline 8 & 17 & 18 & 23 & 28 & 33 \\
\hline 8 & 20 & 19 & 24 & 28 & 34 \\
\hline 9 & 13 & 19 & 25 & 33 & 35 \\
\hline 9 & 19 & 19 & 27 & 35 & 39 \\
\hline 13 & 17 & 19 & 34 & & \\
\hline
\end{tabular}




\section{Dendrimer-substrate equilibration times}




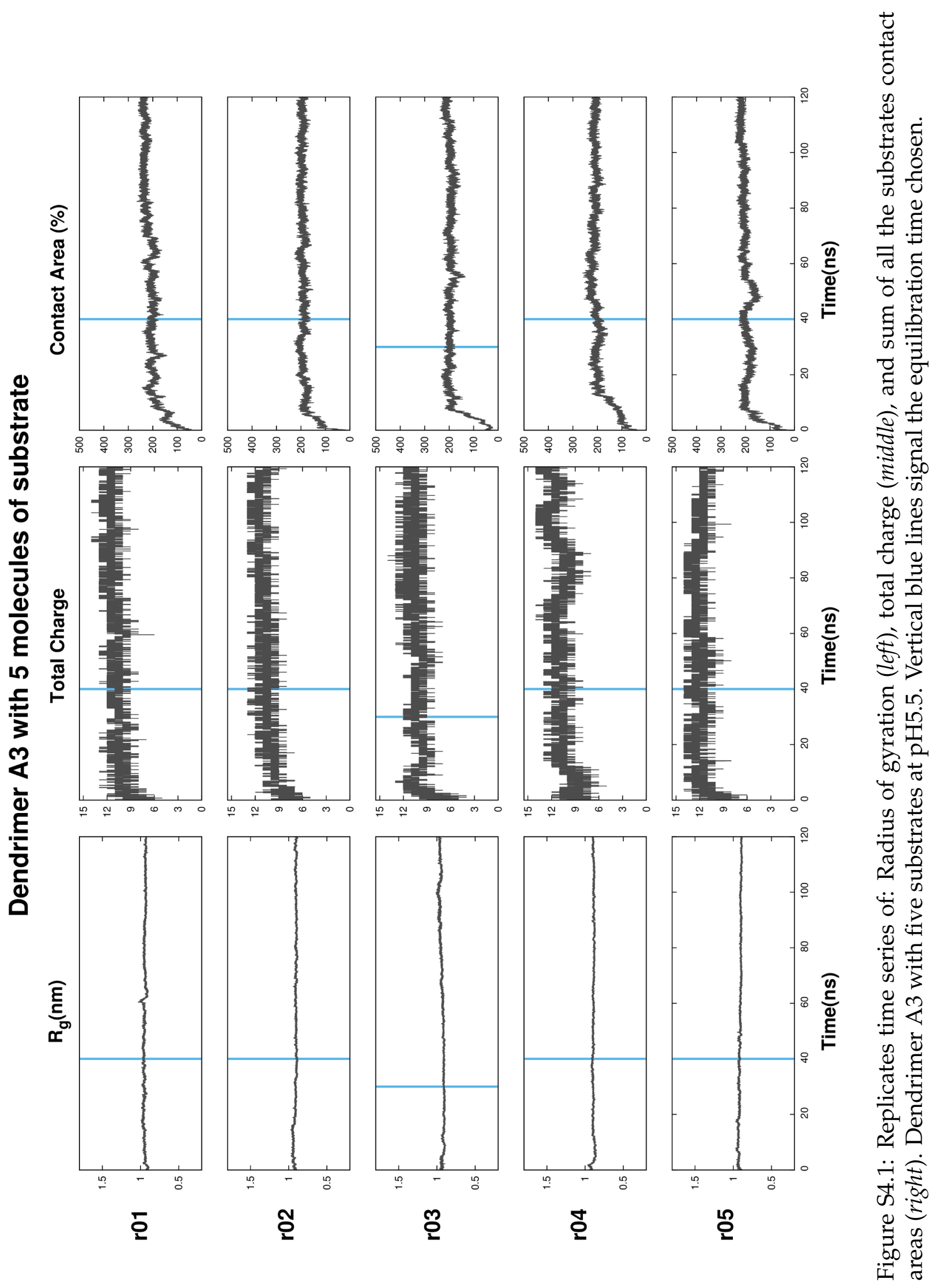




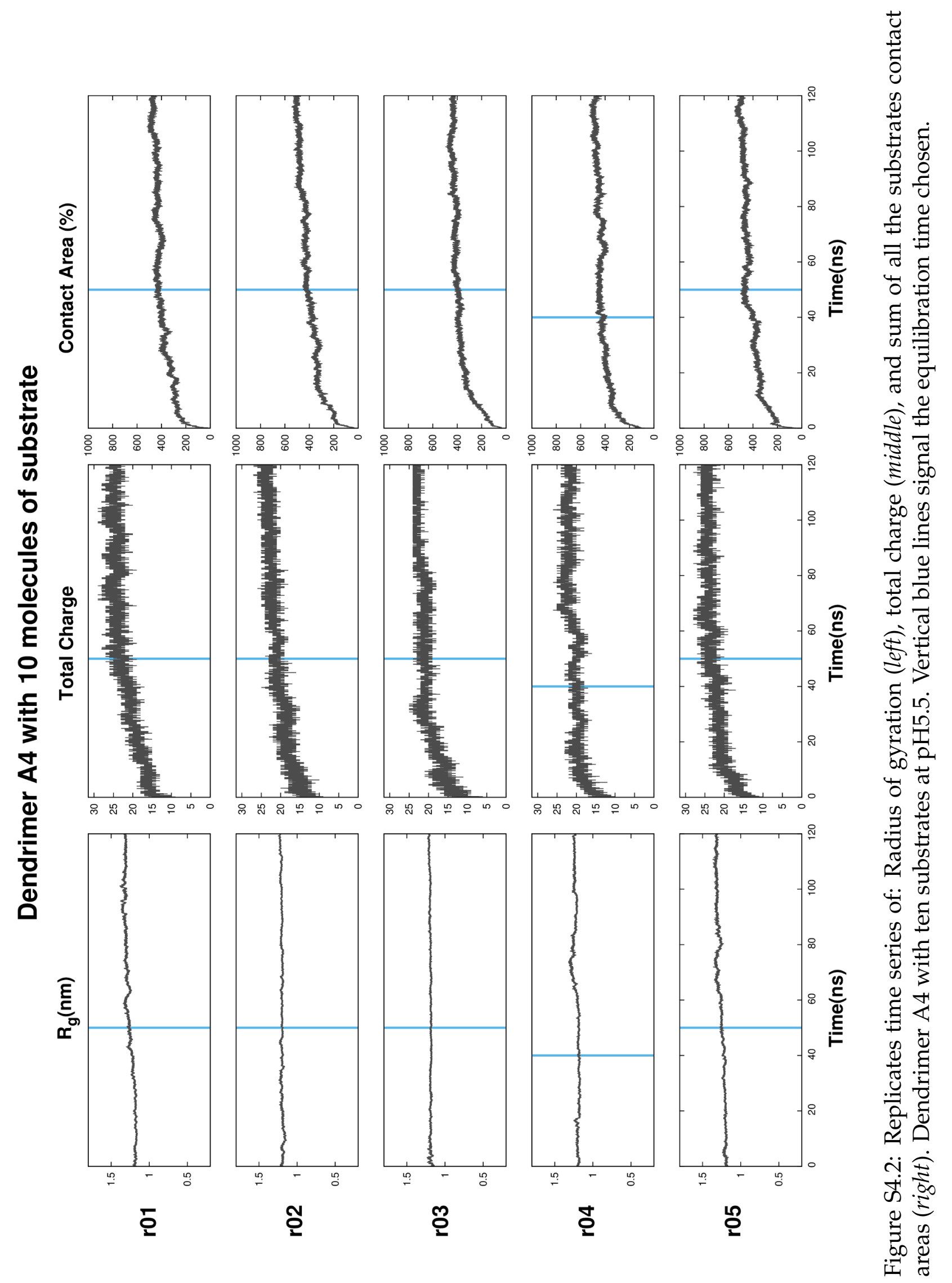




\section{Dendrimer-substrate replicate sampling convergence}
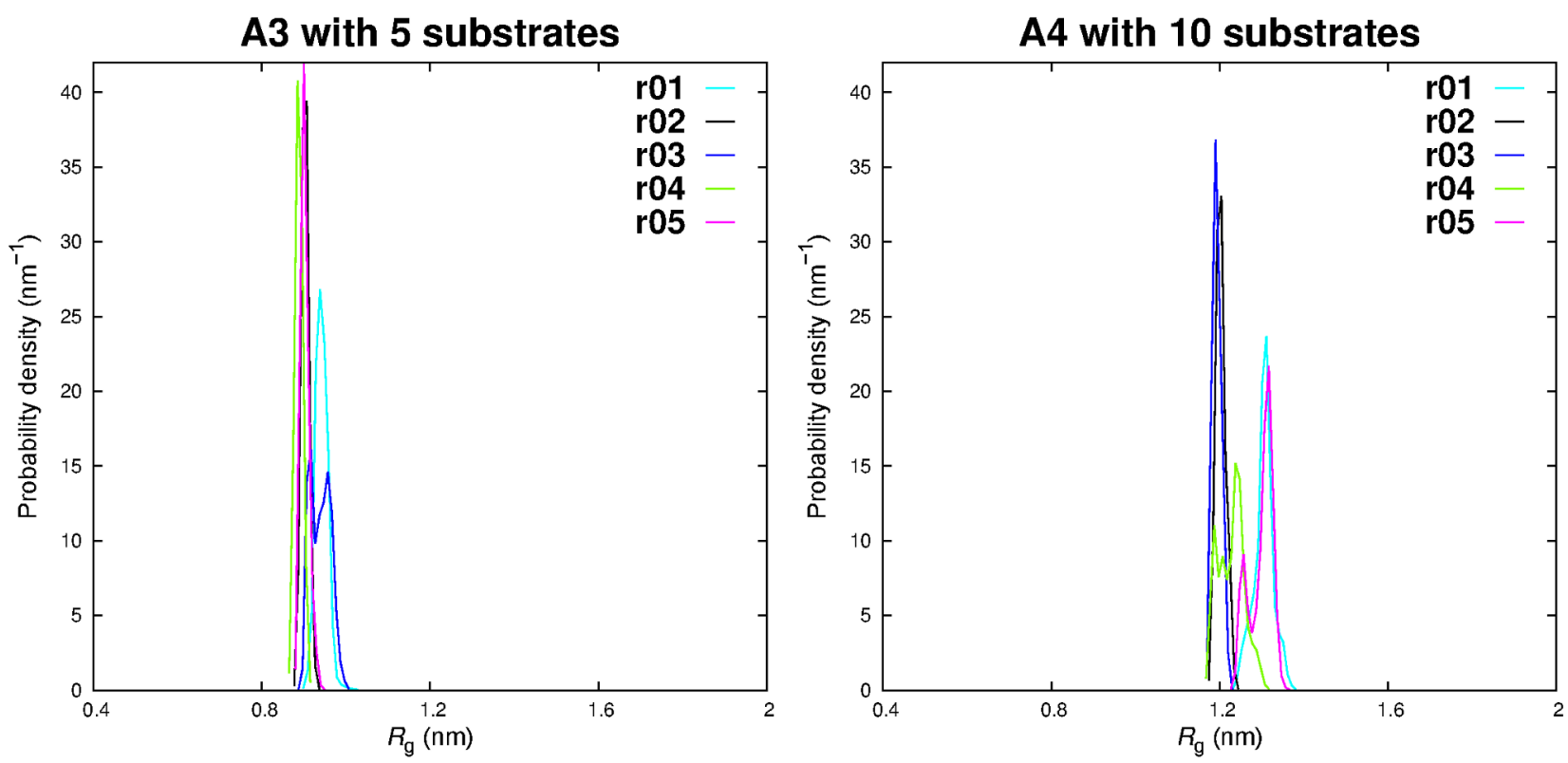

Figure S5.1: Radius of gyration probability density histograms for the different replicates. Dendrimer A3 (left) and dendrimer A4 (right). 


\section{$6 \quad \mathrm{X}-\mathrm{H} \cdots \pi$ test}
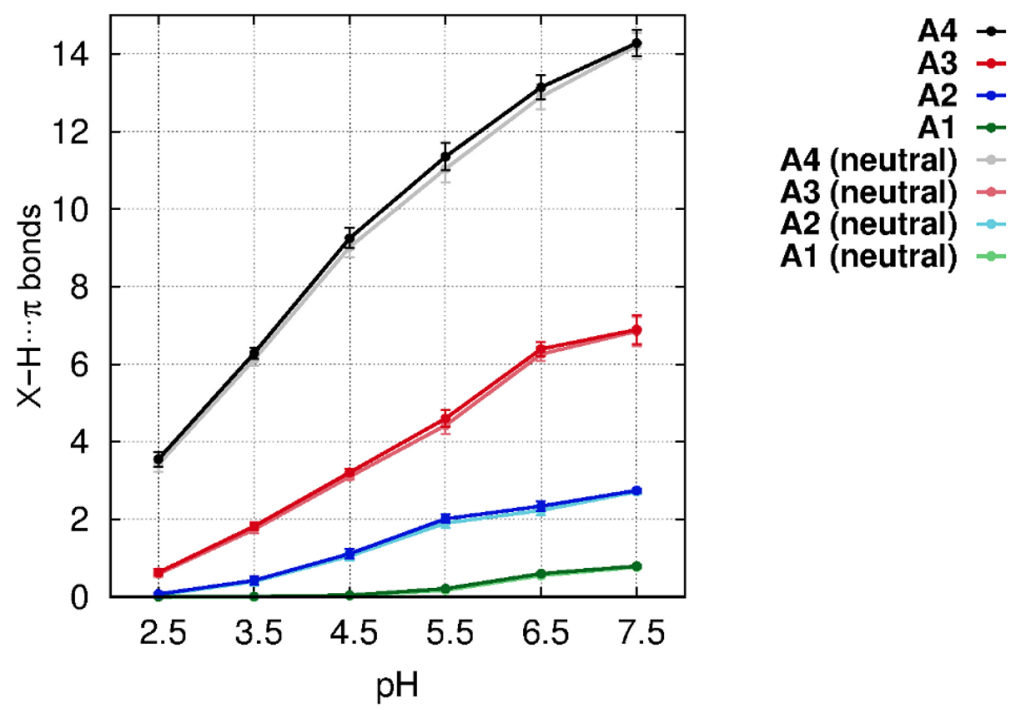

Figure S6.1: We tested the robustness of the criteria employed for $\mathrm{X}-\mathrm{H} \cdots \pi$ bonds by computing $\mathrm{X}-\mathrm{H} \cdots \pi$ interactions considering all histidine residues in the calculations, regardless of their charged form, and performing the same calculations while considering only neutral histidines (neutral lines in the key). 


\section{$7 \quad$ A1 and A2 titration curves}

Dendrimer A1 and A2 were re-synthesized following the procedure described in reference 4 and their titration curves determined using the procedure described in the previous reference. Briefly, the dendrimers were titrated by adding aliquots $20 \mu \mathrm{L}$ of $2 \mathrm{mM}$ $\mathrm{NaOH}$ into a $1 \mathrm{mM}$ aqueous solution of dendrimer, at $25^{\circ} \mathrm{C}$. The experiments were repeated and gave reproducible values within 10\% error. The mass spectra (ESI+) for A1 and A2 are presented in Figures S7.2 and S7.3.

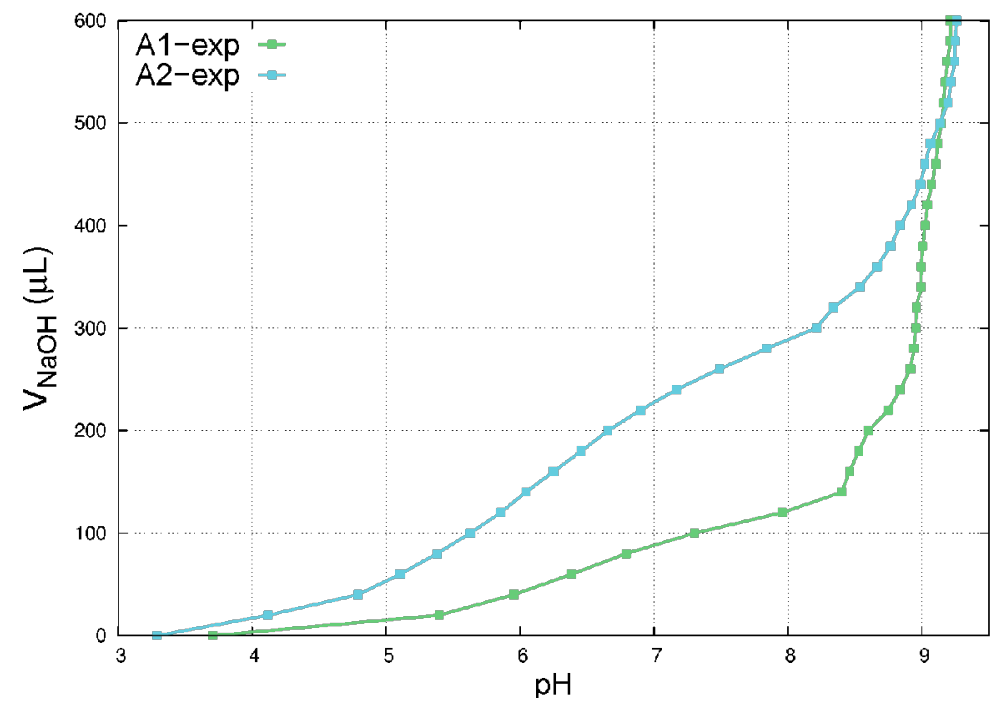

Figure S7.1: Titration curves for A1 and A2. A dendrimer solution (1 mM) was titrated with $20 \mu \mathrm{L}$ aliquots of a $\mathrm{NaOH}$ solution $(2 \mathrm{mM})$. 


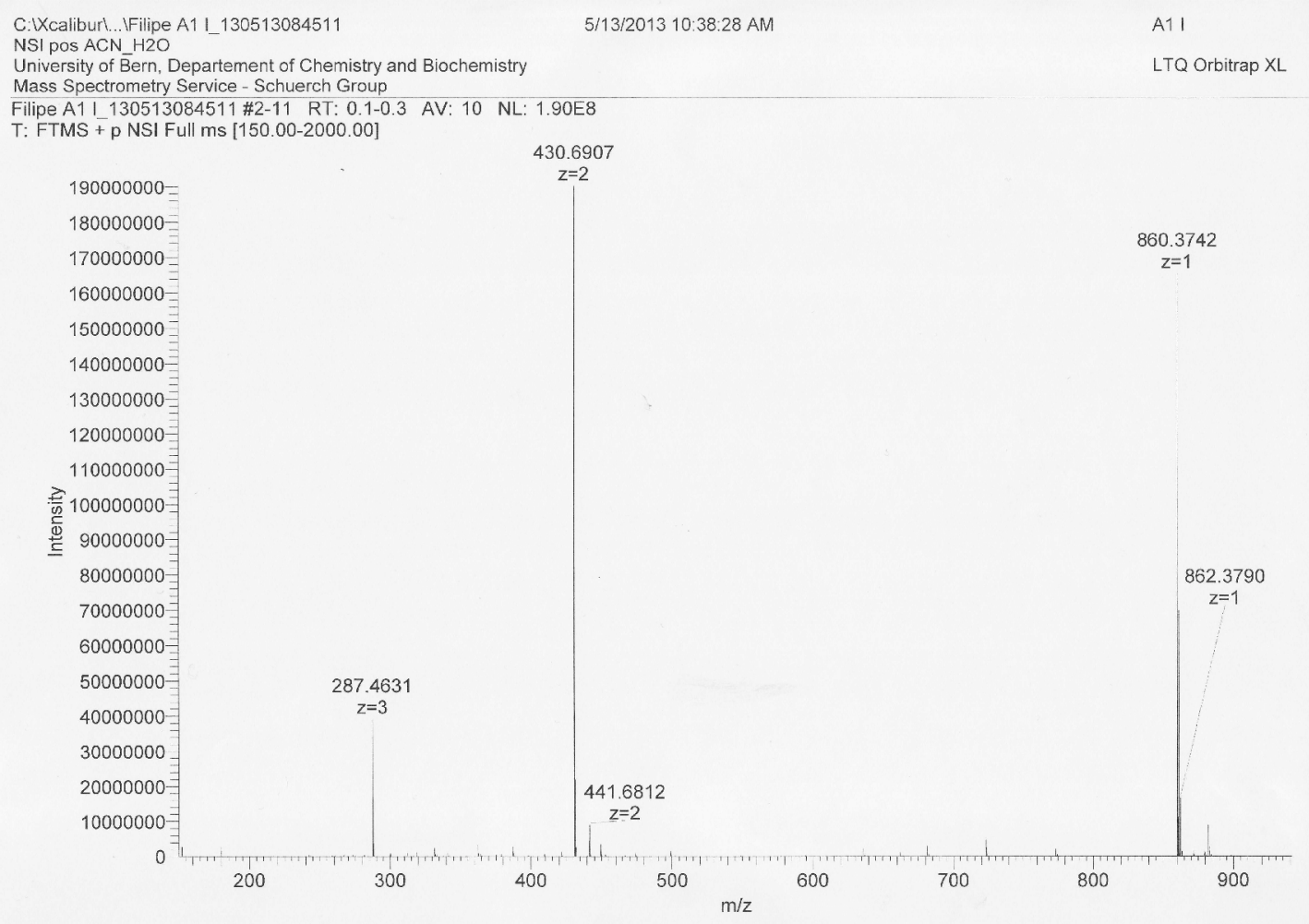

Figure S7.2: Mass Spectra of A1. MS (ESI+) calc. for $\mathrm{C}_{34} \mathrm{H}_{49} \mathrm{~N}_{15} \mathrm{O}_{12}[\mathrm{M}+\mathrm{H}]^{+}$: 859.37, found: 860.37 . 


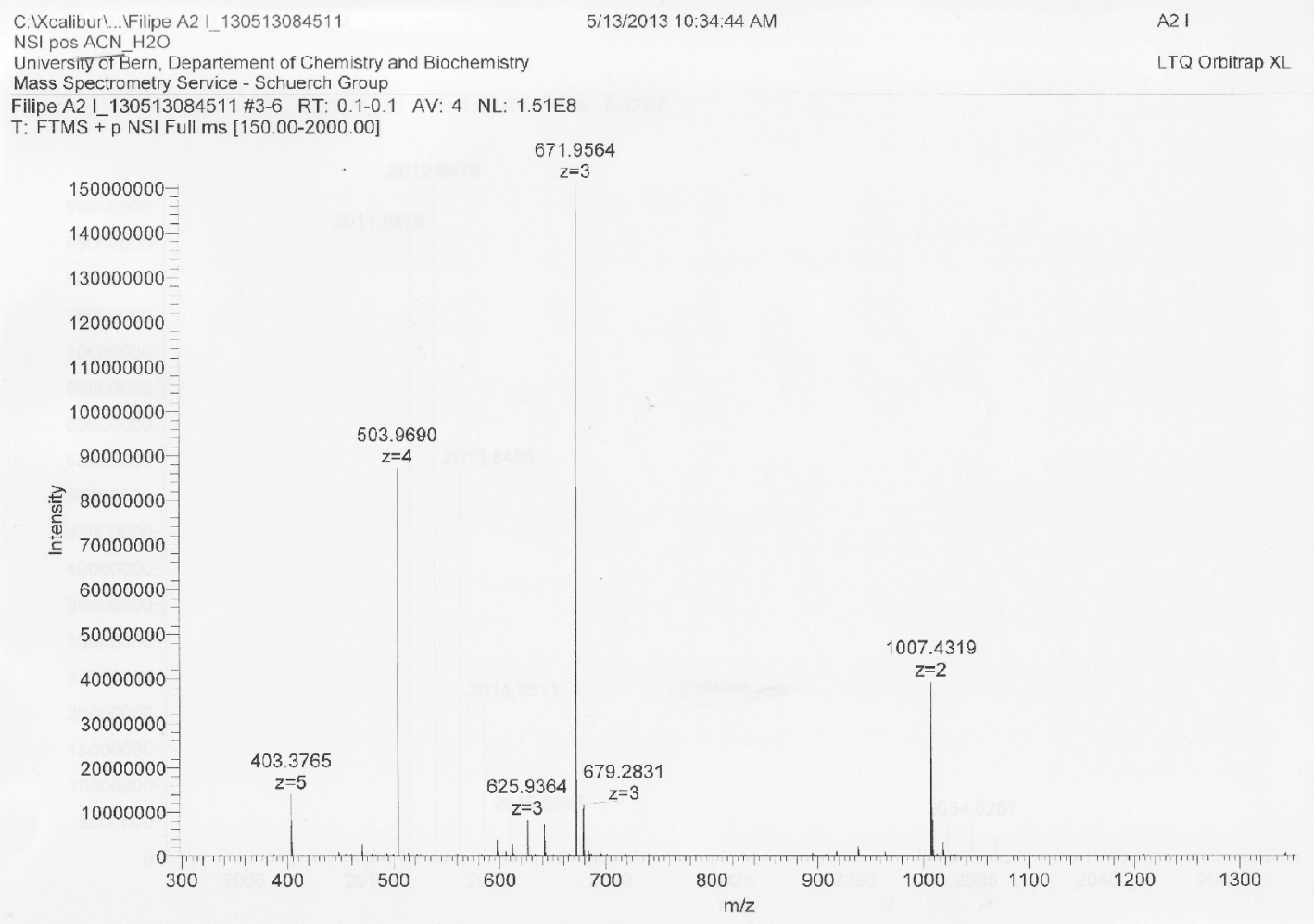

Figure S7.3: Mass Spectra of A2. MS (ESI+) calc. for $\mathrm{C}_{80} \mathrm{H}_{113} \mathrm{~N}_{35} \mathrm{O}_{28}[\mathrm{M}+\mathrm{H}]^{+}$: 2011.84, found: 2012.85 . 


\section{$8 R_{\mathrm{g}}$ histograms at different $\mathrm{pH}$ values}

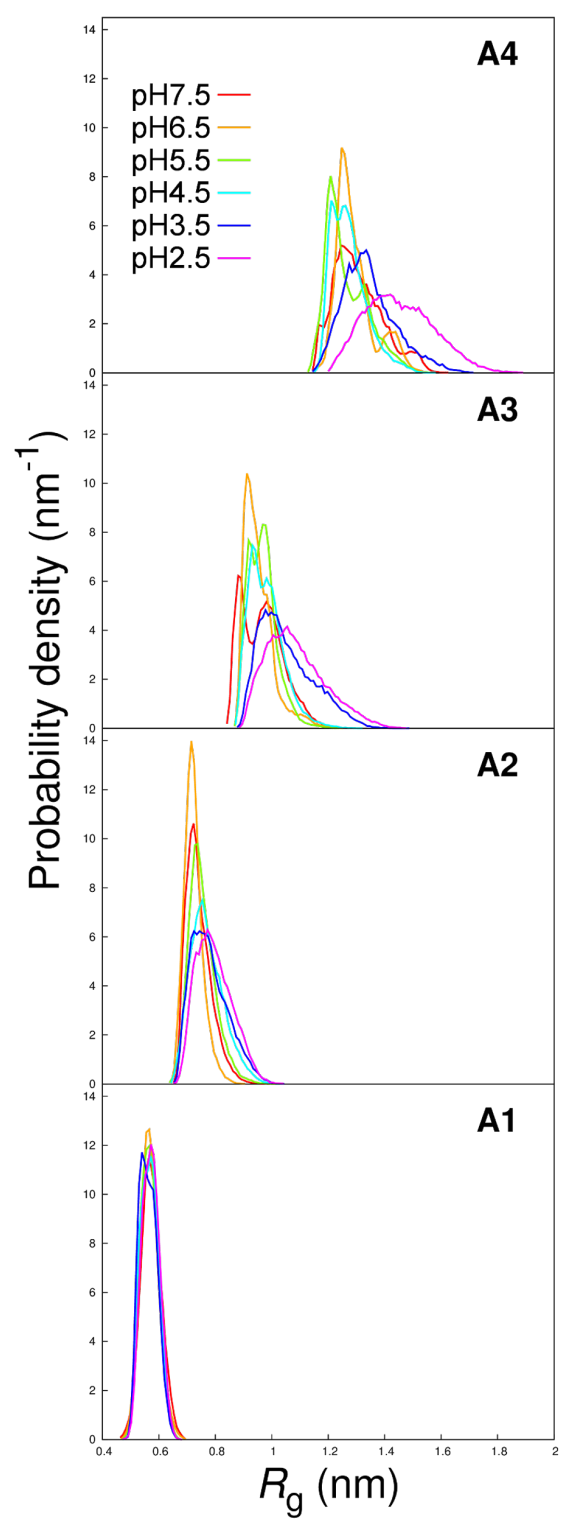

Figure S8.1: $R_{\mathrm{g}}$ probability density histograms for $\mathrm{A} 1, \mathrm{~A} 2, \mathrm{~A} 3$ and $\mathrm{A} 4$ at different $\mathrm{pH}$ values. 


\section{Average SASA at different $\mathrm{pH}$ values}

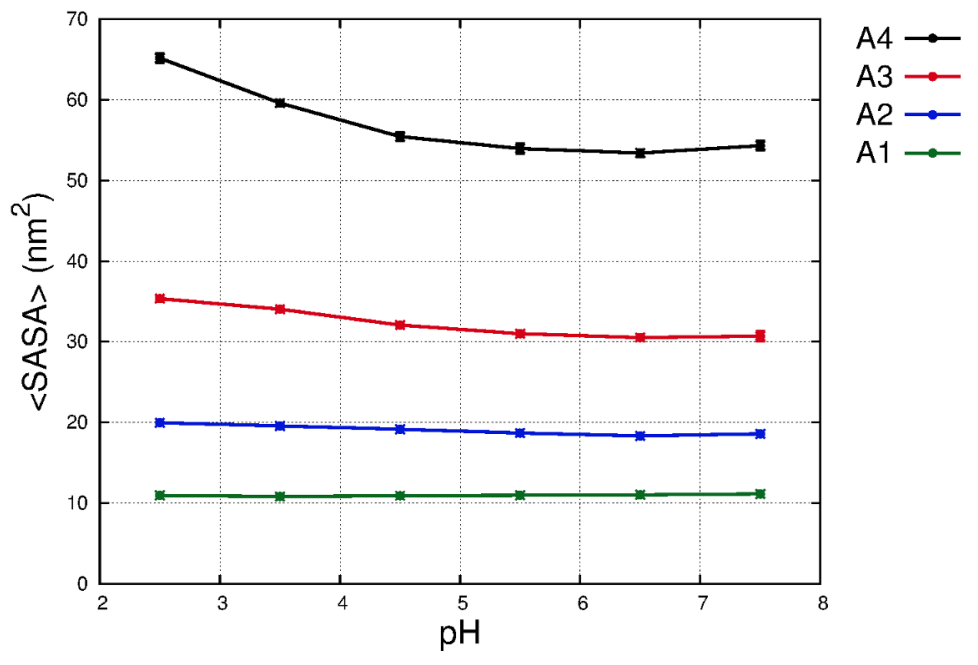

Figure S9.1: Average solvent accessible surface area of the dendrimer at different $\mathrm{pH}$ values. 


\section{Energy landscapes: A1, A2 and A3}
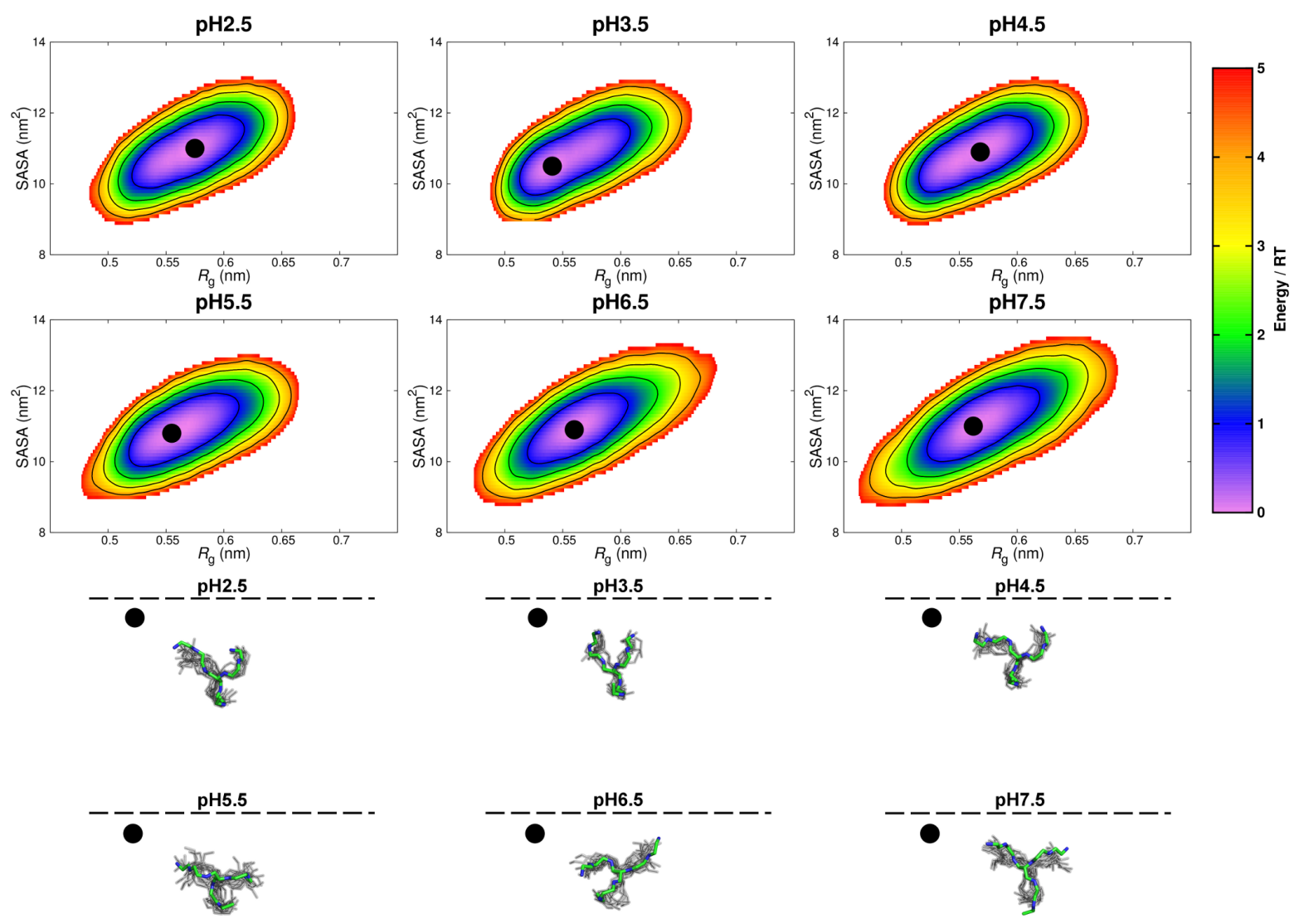

Figure S10.1: Dendrimer A1 energy profiles and lowest energy conformers at different $\mathrm{pH}$ values. The energy profiles use $R_{\mathrm{g}}$ and SASA as structural coordinates. In the energy profiles the lowest energy cluster is represented as a black dot. For the different $\mathrm{pH}$ values pictures of the 21 lowest energy conformers of each energy cluster are shown, with the lowest energy structure from each cluster highlighted (only the peptidic backbone is represented using green for carbons and blue for nitrogen atoms) and superimposed on the remaining 20 structures (in gray). 

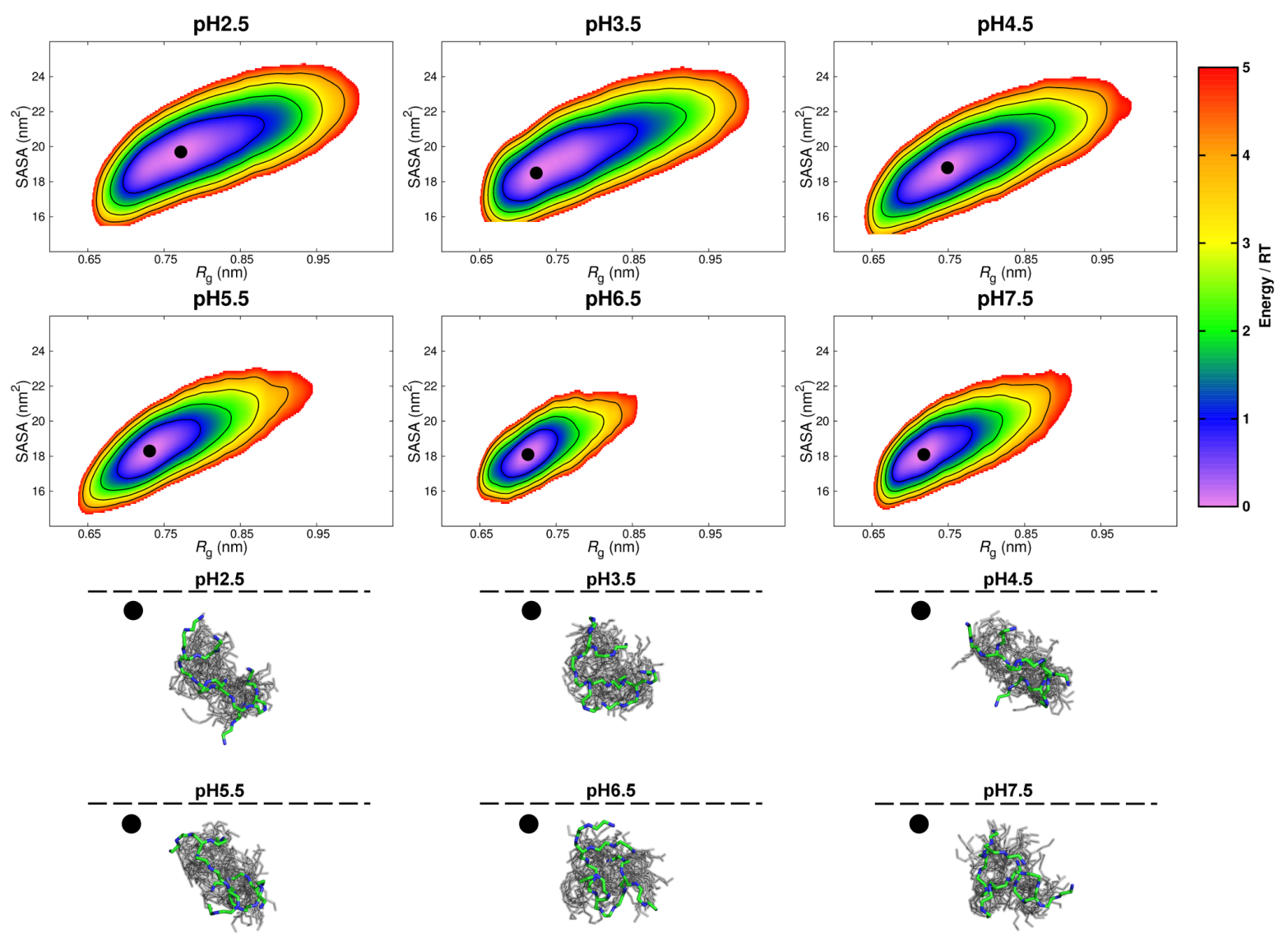

Figure S10.2: Dendrimer A2 energy profiles and lowest energy conformers at different $\mathrm{pH}$ values. The energy profiles use $R_{\mathrm{g}}$ and SASA as structural coordinates. In the energy profiles the lowest energy cluster is represented as a black dot. For the different $\mathrm{pH}$ values pictures of the 21 lowest energy conformers of each energy cluster are shown, with the lowest energy structure from each cluster highlighted (only the peptidic backbone is represented using green for carbons and blue for nitrogen atoms) and superimposed on the remaining 20 structures (in gray). 

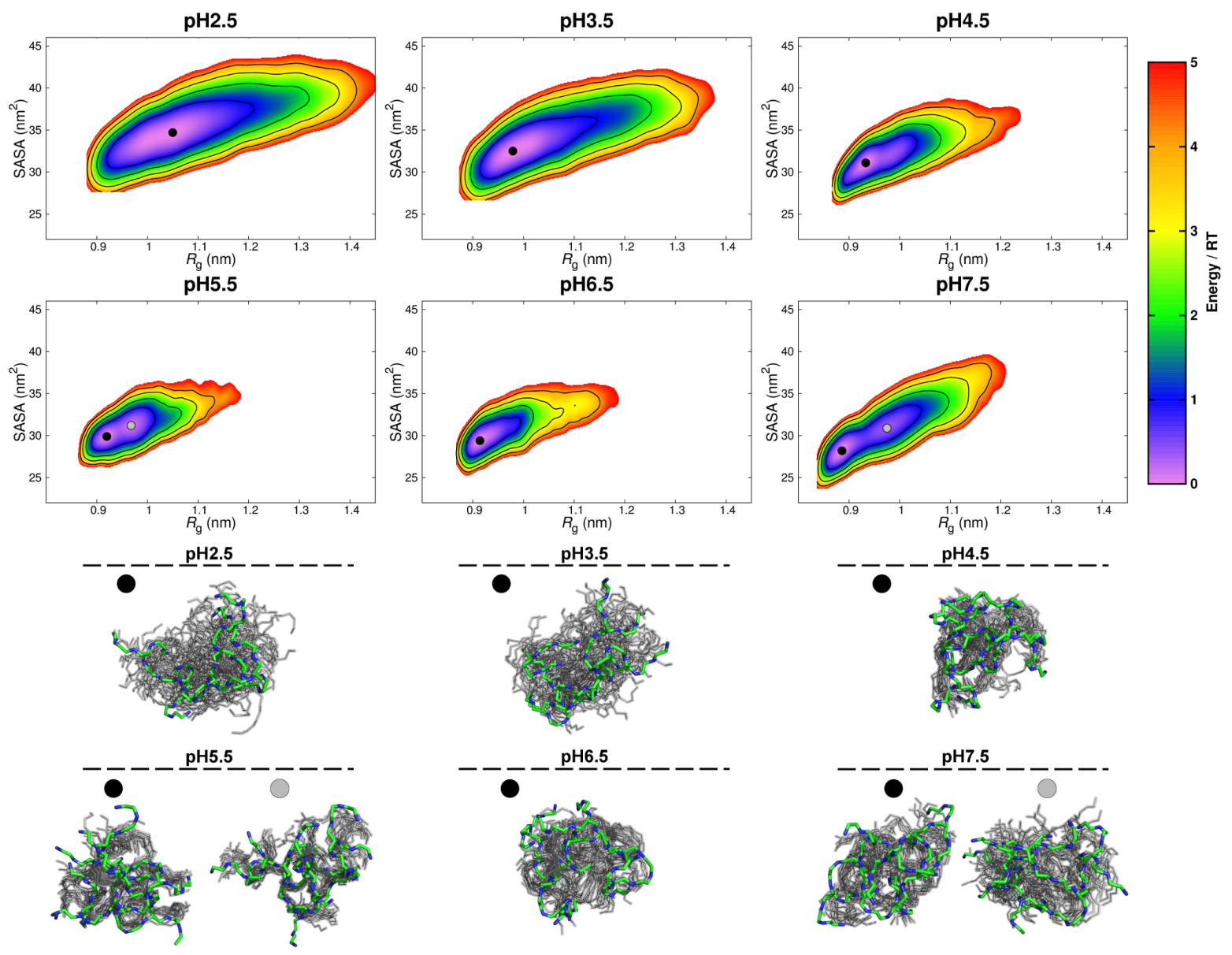

Figure S10.3: Dendrimer A3 energy profiles and lowest energy conformers at different $\mathrm{pH}$ values. The energy profiles use $R_{\mathrm{g}}$ and SASA as structural coordinates. In the energy profiles the lowest energy cluster is represented as a black dot. For the different $\mathrm{pH}$ values pictures of the 21 lowest energy conformers of each energy cluster are shown, with the lowest energy structure from each cluster highlighted (only the peptidic backbone is represented using green for carbons and blue for nitrogen atoms) and superimposed on the remaining 20 structures (in gray). 


\section{$11 \pi-\pi$ interactions}
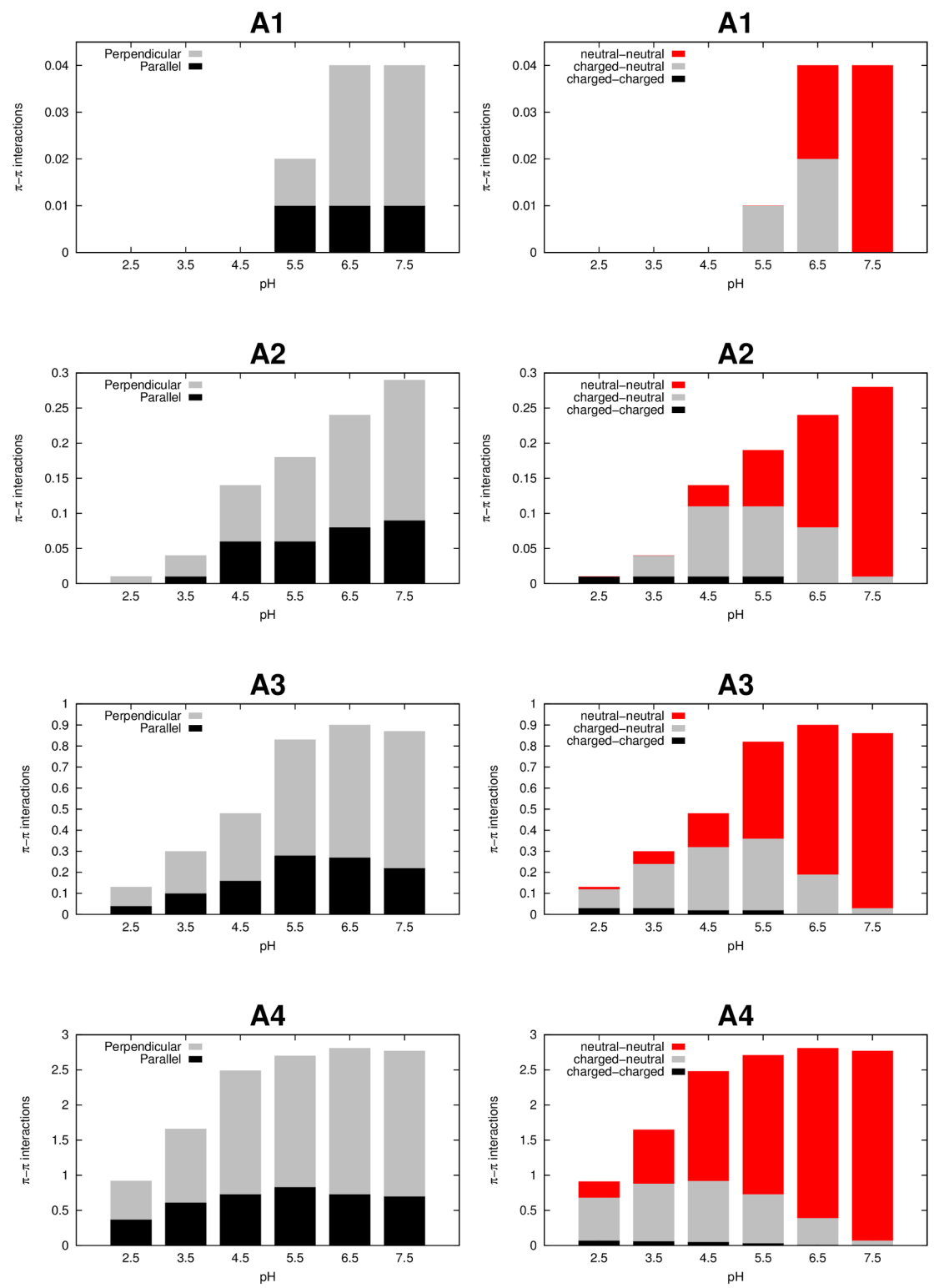

Figure S11.1: Distribution of $\pi-\pi$ stacking by type (left) and histidine charged states (right). The sum the values in each column yields the average number of $\pi-\pi$ interactions at each $\mathrm{pH}$. 


\section{Hydrogen Bonds: MainChain vs. SideChain}
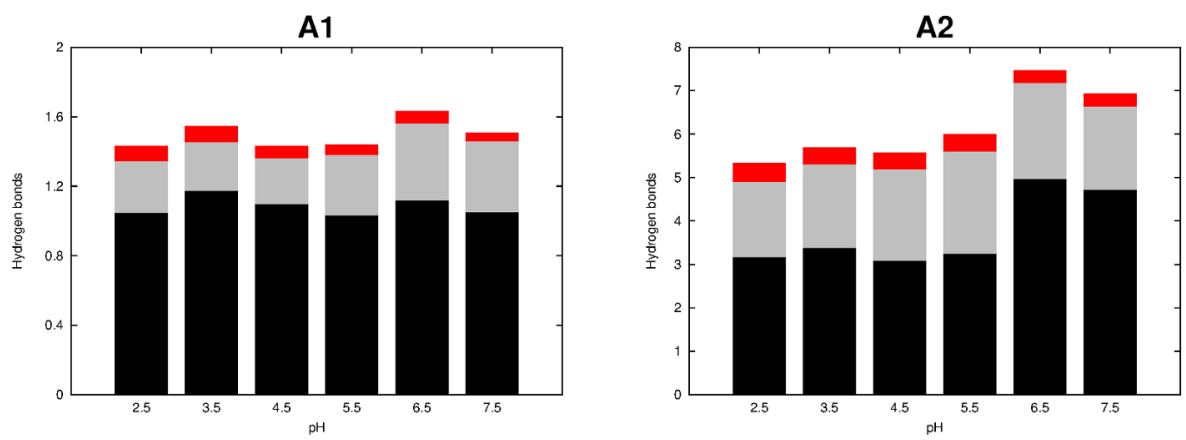

Donor Acceptor

Side

Side

Main Side

Main

Main
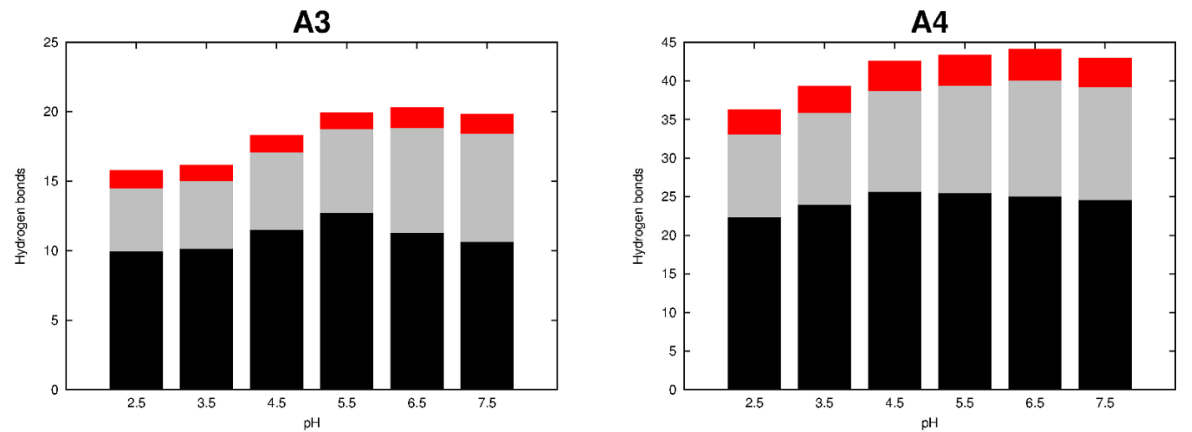

Figure S12.1: Distribution of HB by donors and acceptors of the dendrimers main-chain or side-chain. The sum the values in each column yields the average number of $\mathrm{HB}$ at each $\mathrm{pH}$. 


\section{Histidines protonation curves}
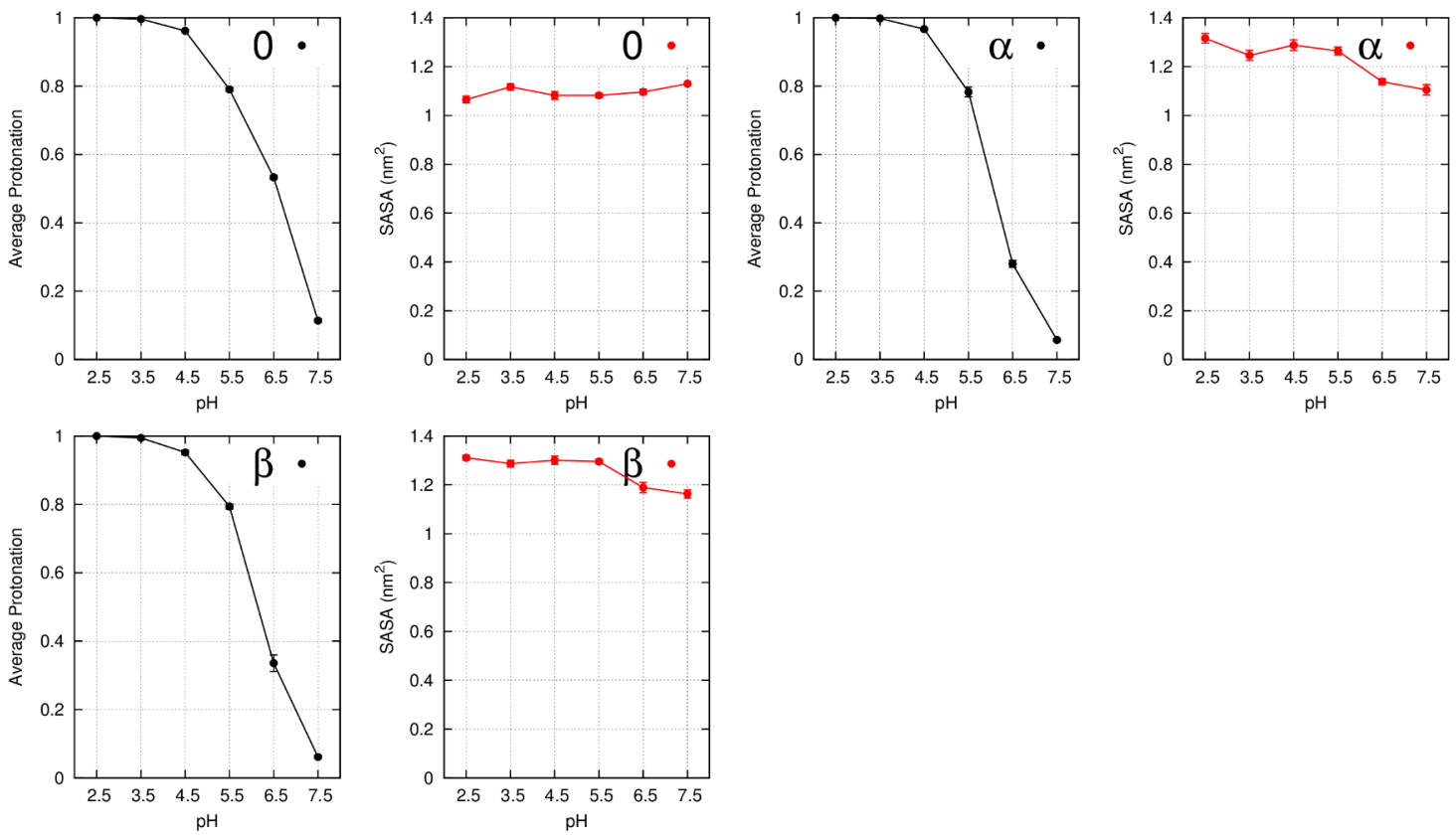

Figure S13.1: Dendrimer A1. Histidines average proton occupancy and imidazole ring solvent accessible surface area curves. 

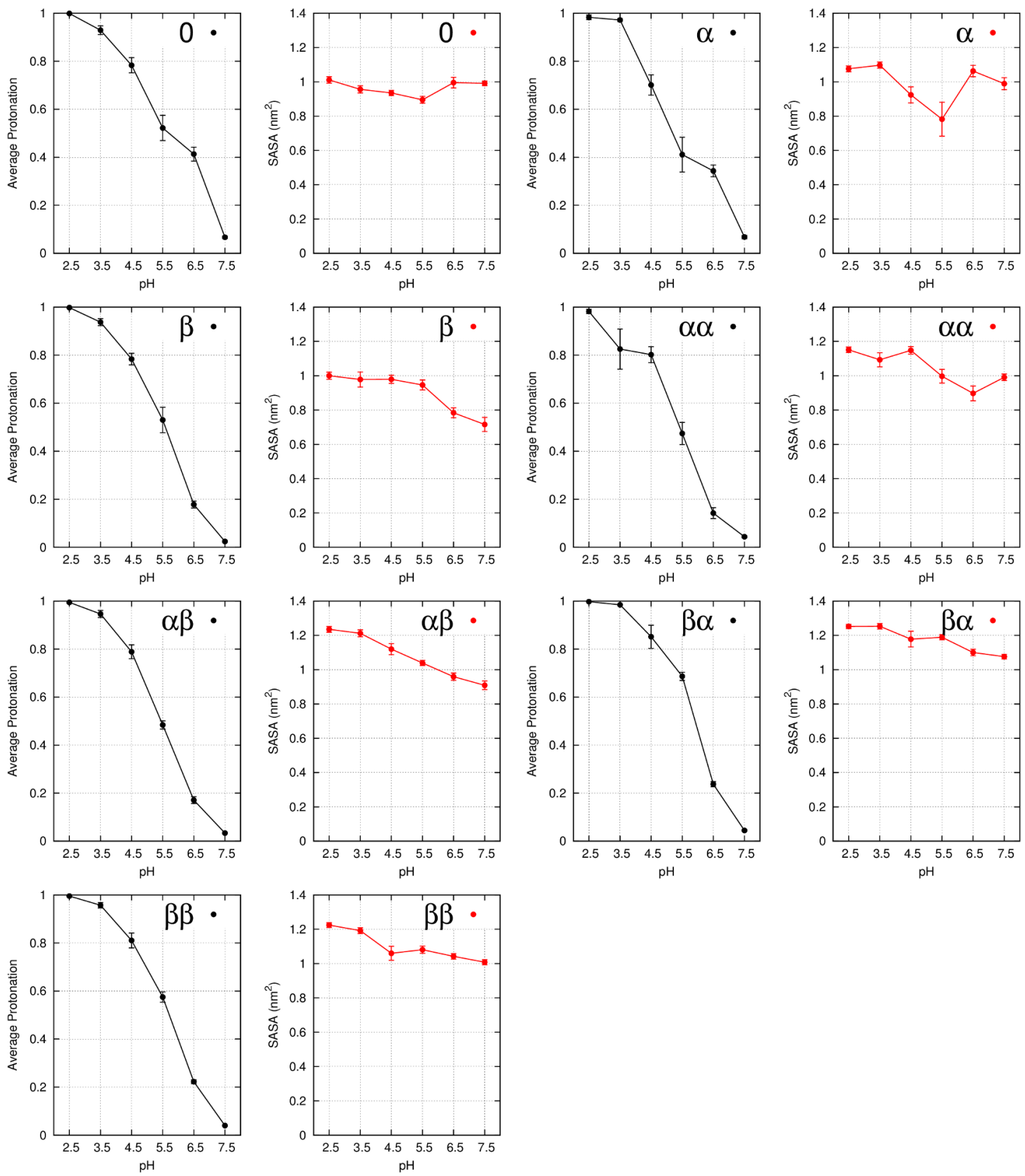

Figure S13.2: Dendrimer A2. Histidines average proton occupancy and imidazole ring solvent accessible surface area curves. 

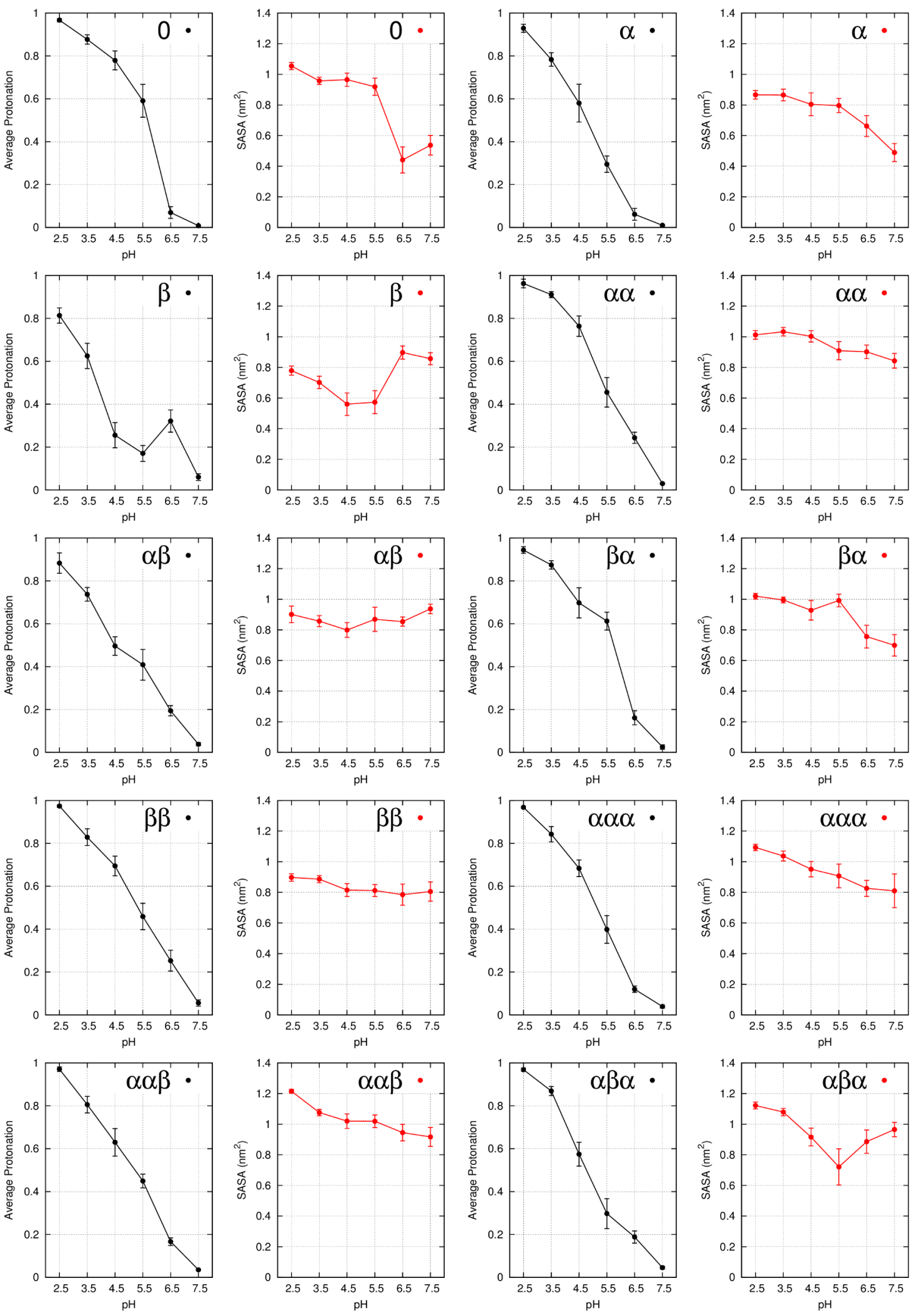

Figure S13.3: Dendrimer A3. Histidines average proton occupancy and imidazole ring solvent accessible surface area curves. 

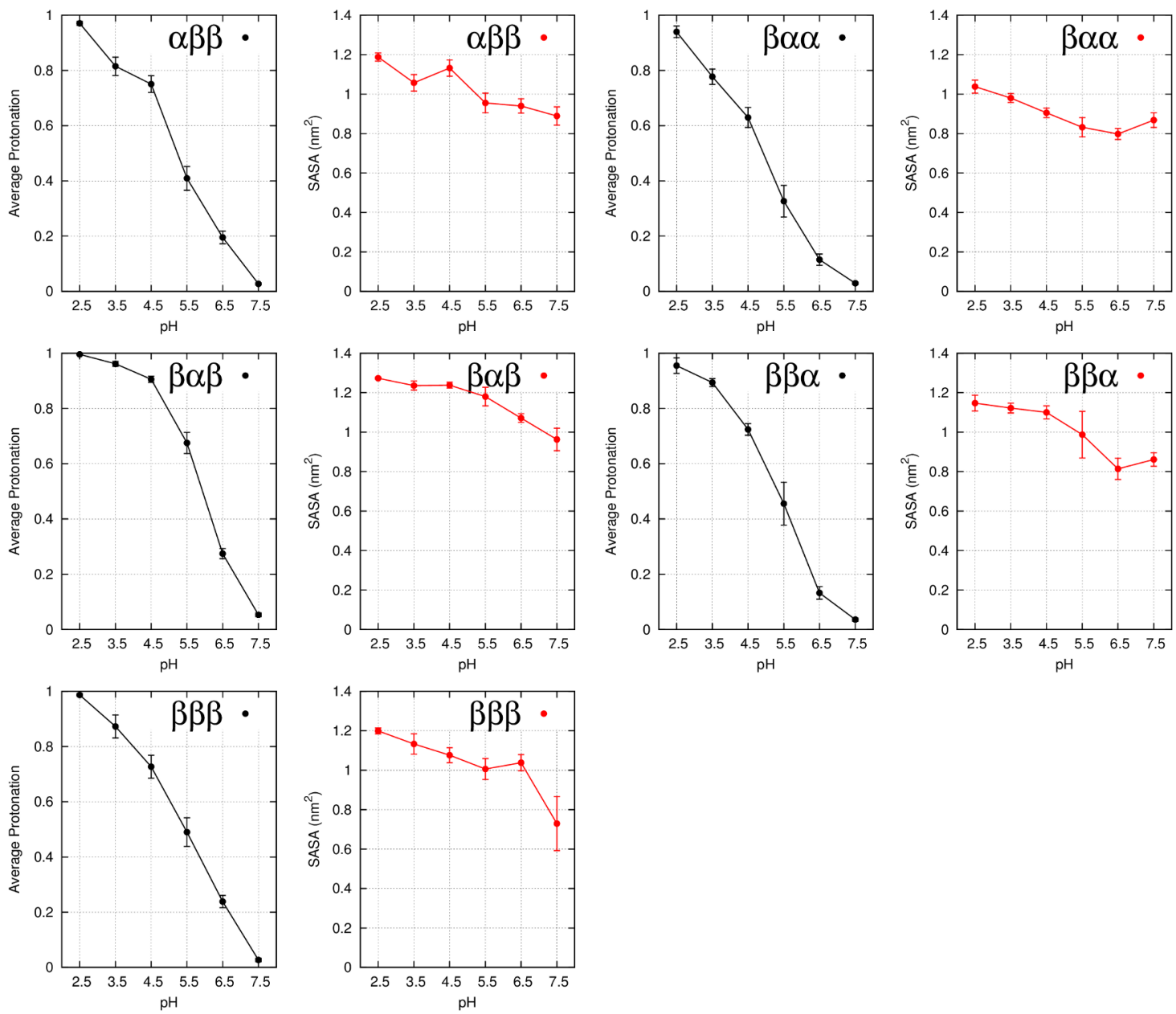

Figure S13.4: Dendrimer A3. Histidines average proton occupancy and imidazole ring solvent accessible surface area curves. 

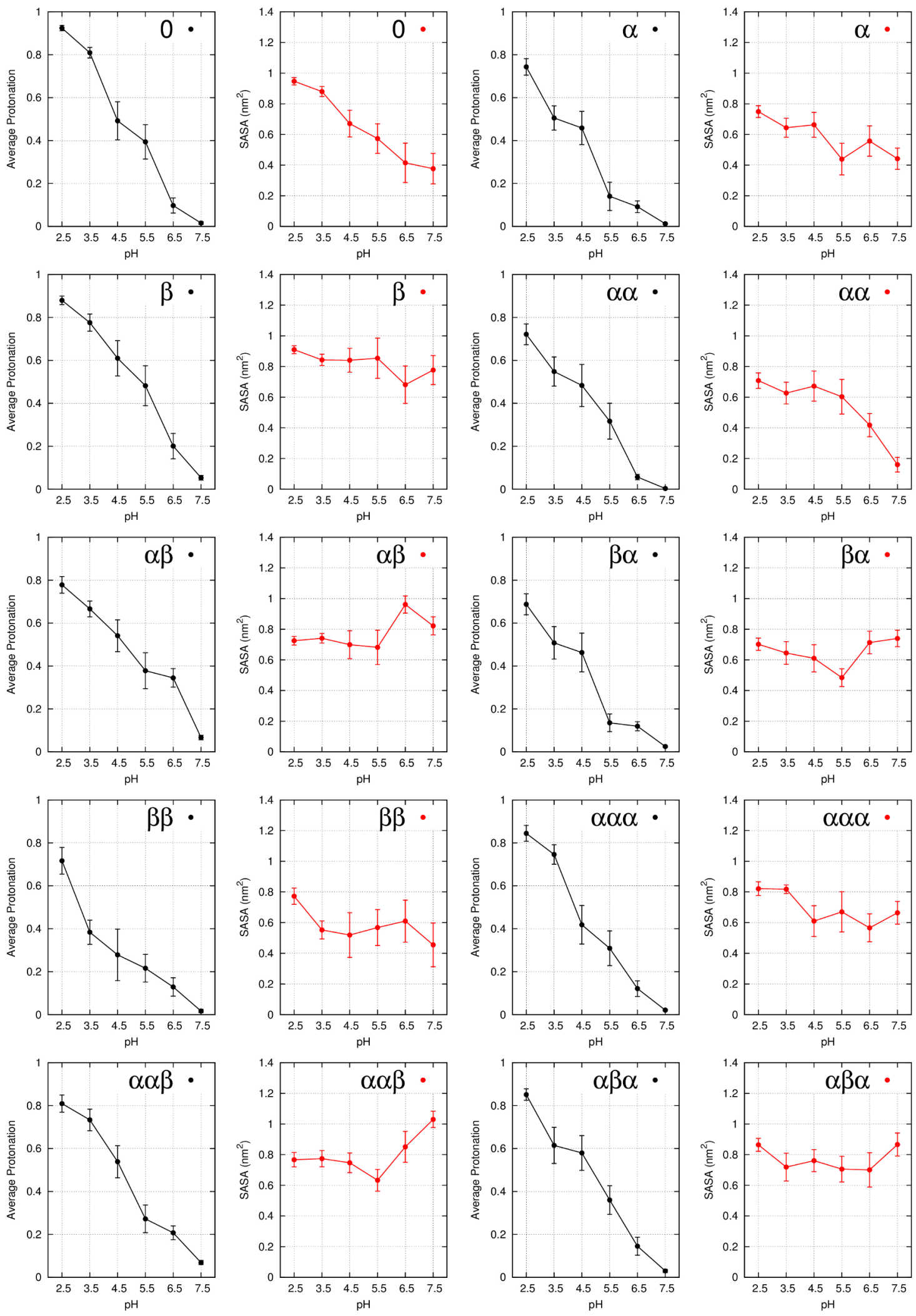

Figure S13.5: Dendrimer A4. Histidines average proton occupancy and imidazole ring solvent accessible surface area curves. 

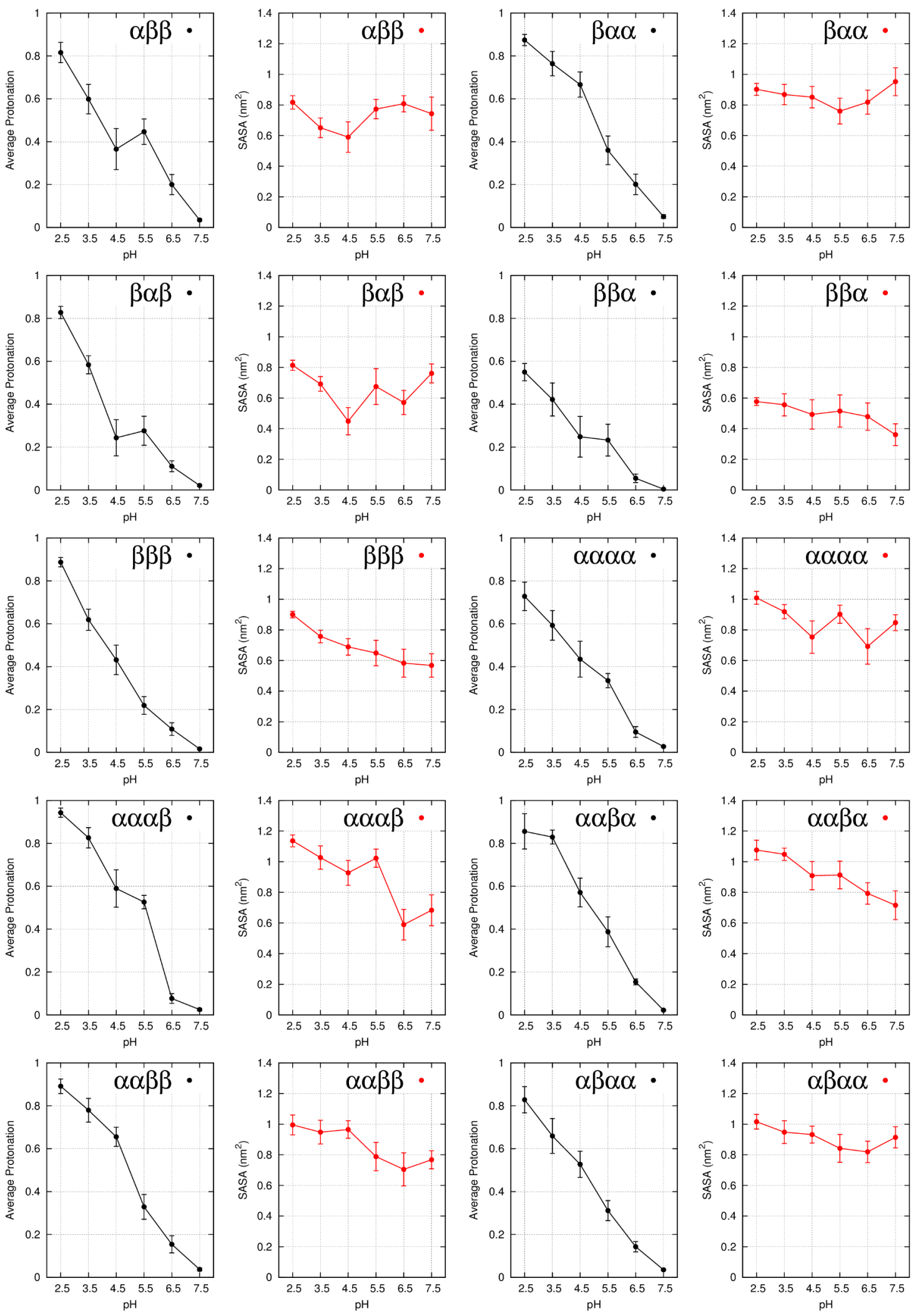

Figure S13.6: Dendrimer A4. Histidines average proton occupancy and imidazole ring solvent accessible surface area curves. 

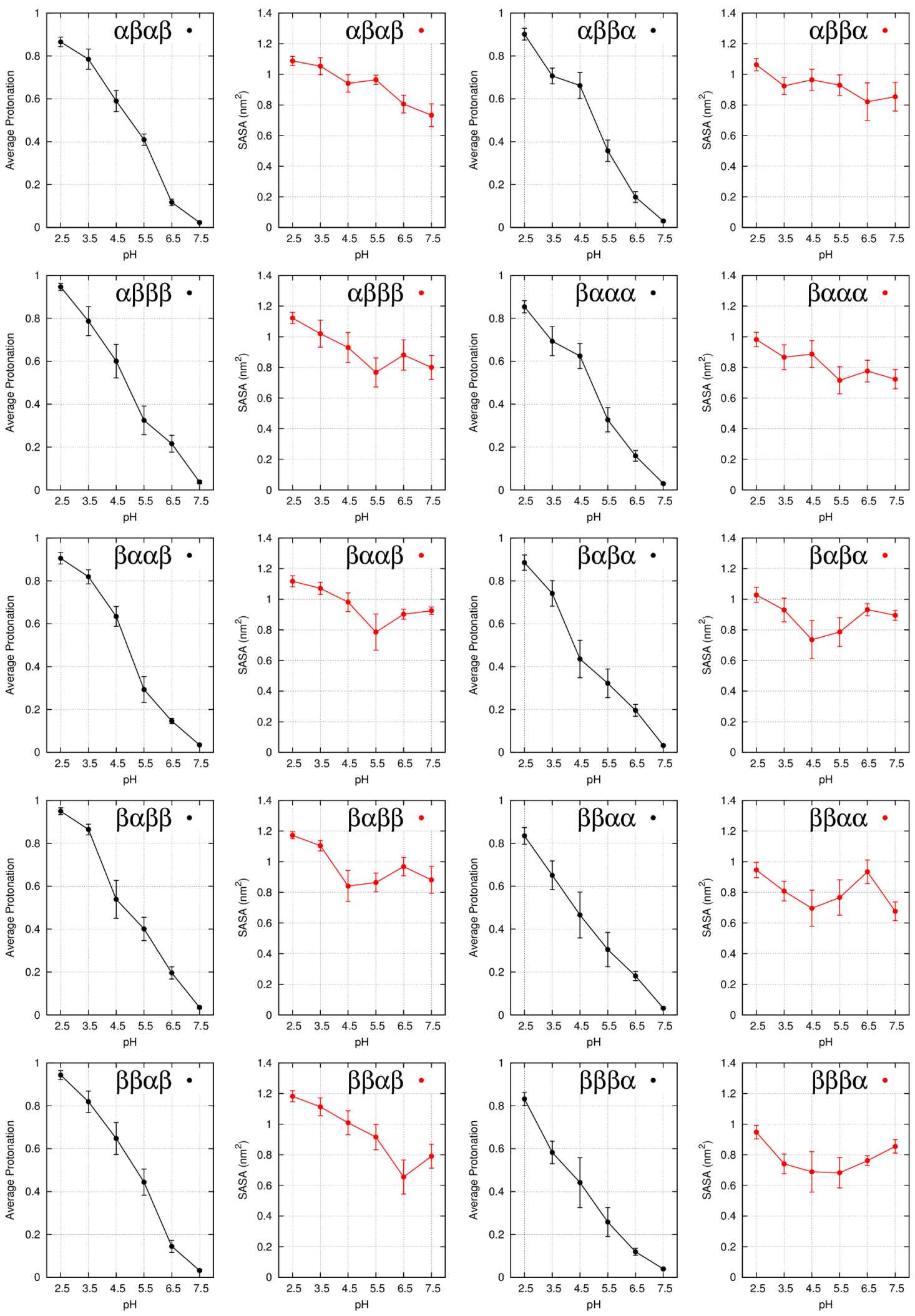

Figure S13.7: Dendrimer A4. Histidines average proton occupancy and imidazole ring solvent accessible surface area curves. 

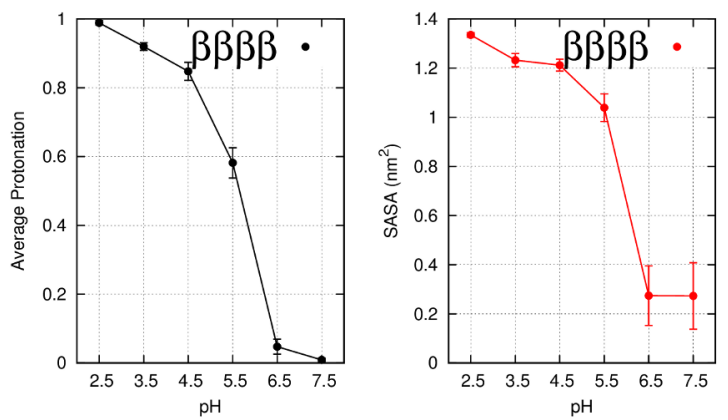

Figure S13.8: Dendrimer A4. Histidines average proton occupancy and imidazole ring solvent accessible surface area curves. 


\section{Dendrimer-Substrate contact area}

The percentage of contact area between a dendrimer and a molecule of substrate was calculated as:

$$
\text { ContactArea }=\left(1-\frac{\text { SASA }_{\text {complex }}}{\text { SASA }_{\text {free }}}\right) \times 100
$$

where, SASA $_{\text {complex }}$ is the solvent accessible surface area of the substrate when considering the dendrimer-substrate complex and $\mathrm{SASA}_{\text {free }}$ is the total solvent accessible surface area of the substrate alone. Hence, the ContactArea is the percentage of substrate SASA that is in contact with the dendrimer surface. This value was computed for each substrate in each frame. SASA values were obtained using the method described in reference 5 using a $1.4 \AA$ A spherical probe. 


\section{Dendrimer-substrate interaction tests}

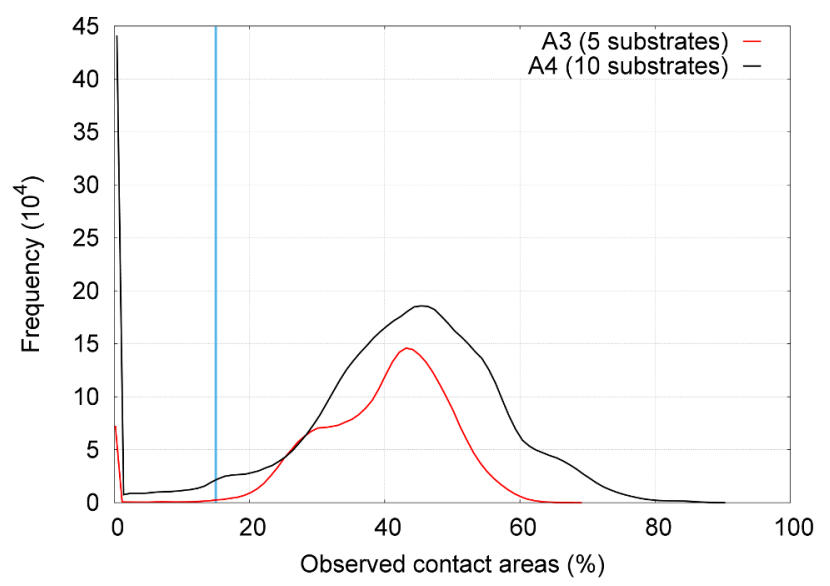

Figure S15.1: Distribution of dendrimer-substrate contact areas. The selected cutoff (15\% of contact area) is displayed as a blue line.
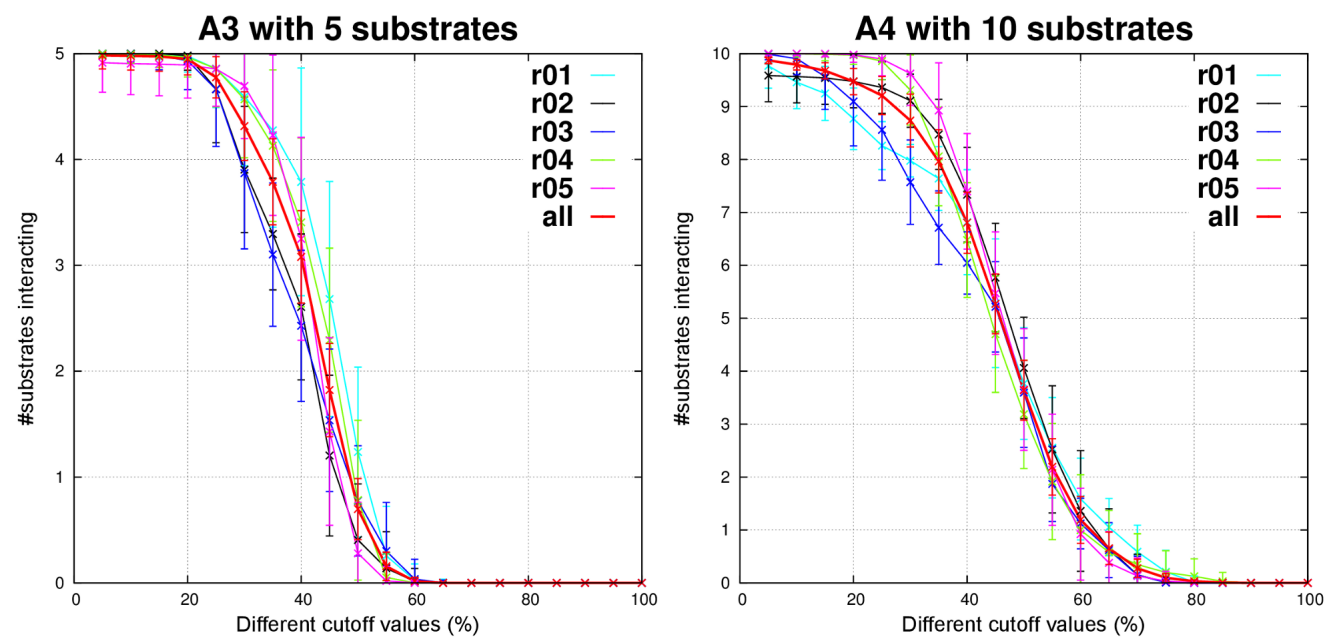

Figure S15.2: Number of substrate molecules considered as interacting with the dendrimer when testing different cutoff values for the contact area. Curves for each replicate and for the entire concatenated trajectories (all) are presented. 


\section{Dendrimer-Substrate complexes}
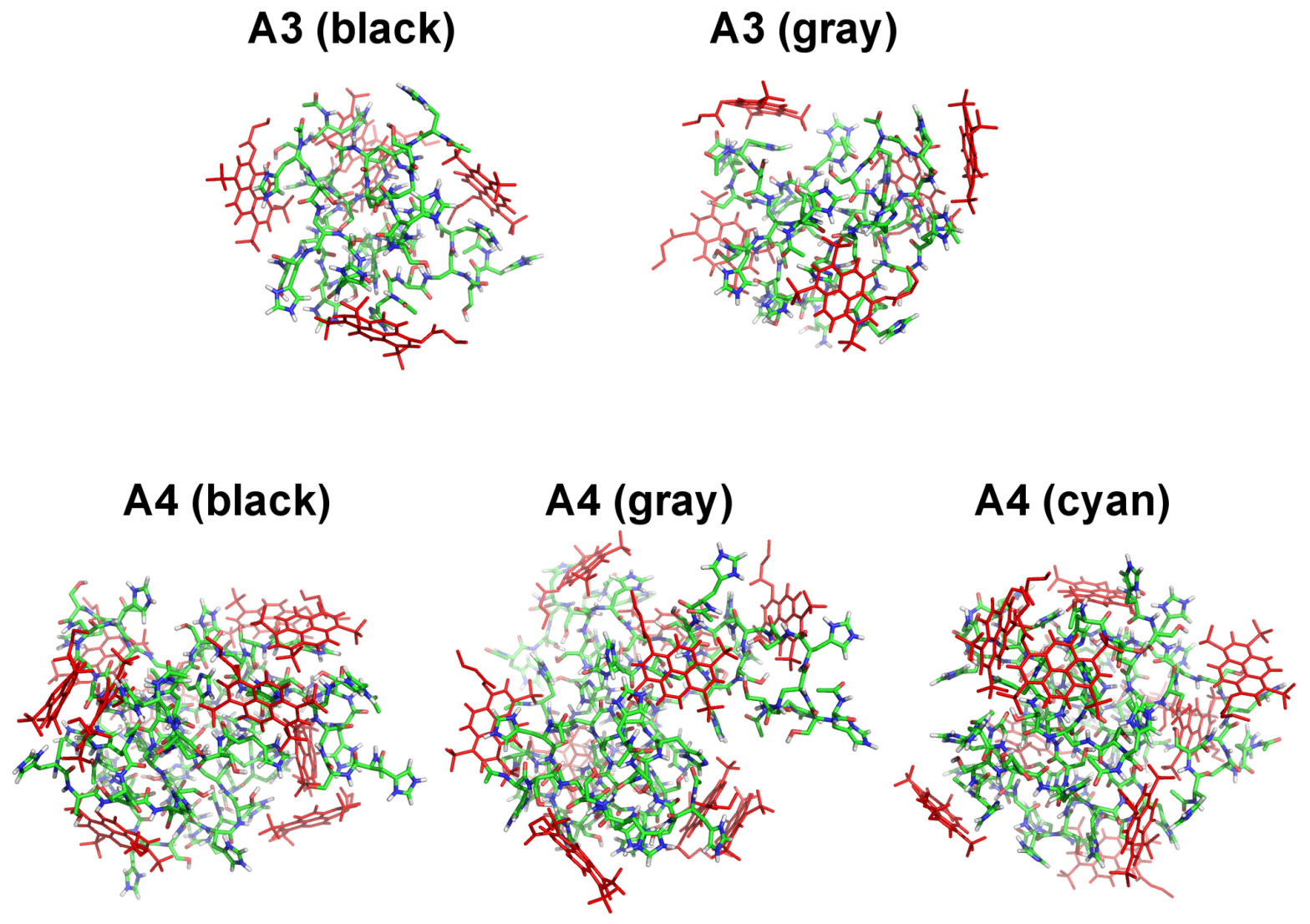

Figure S16.1: Examples of dendrimer-substrate complexes. The images correspond to the lowest energy conformations of the 2D landscapes of A3 and A4 in the presence of 5 and 10 substrate molecules, respectively (Figure 9 in the article). Substrate molecules are shown in red. 

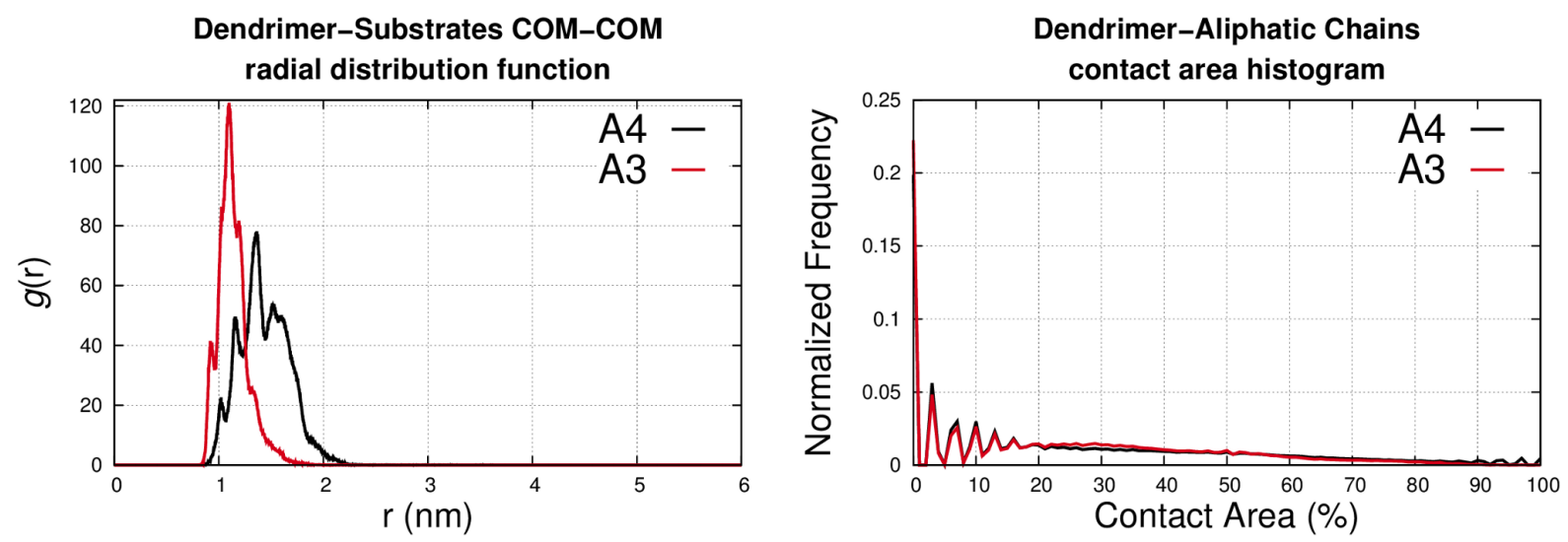

Figure S16.2: (Left) Dendrimer-substrates COM-COM radial distribution functions. (Right) Histogram of the contact area between the aliphatic chains of the substrate molecules and the dendrimer; binsize $=1 \%$. 


\section{A3: Replicate 1}
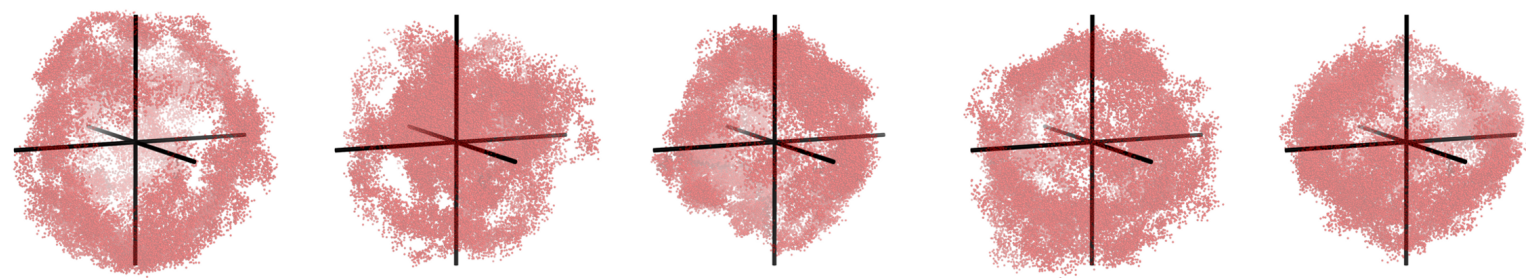

\section{A3: Replicate 2}
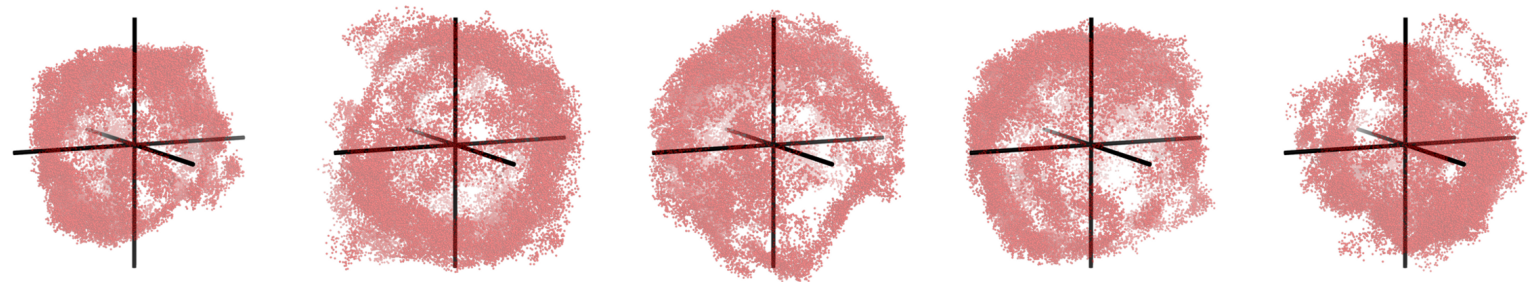

\section{A3: Replicate 3}
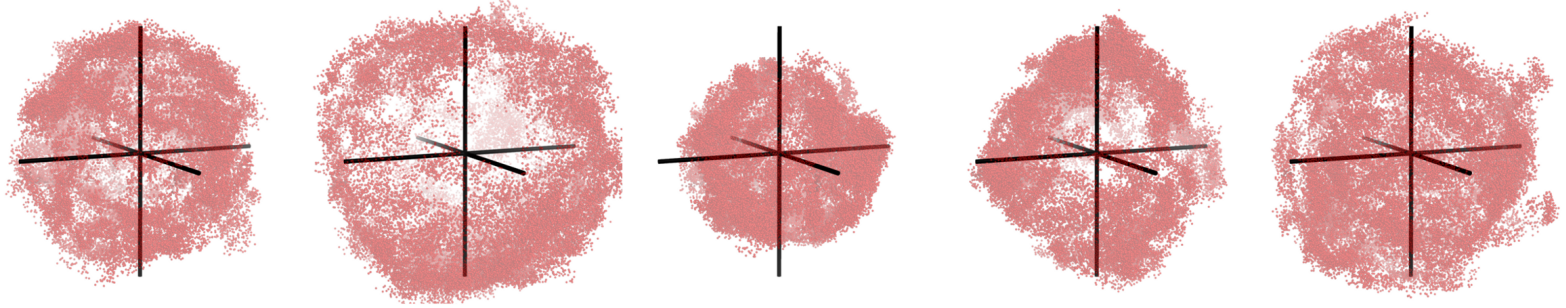

\section{A3: Replicate 4}
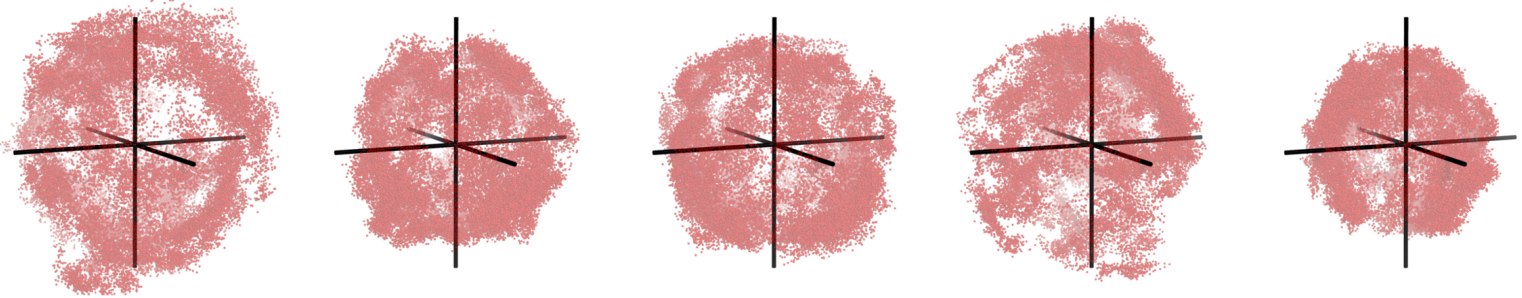

\section{A3: Replicate 5}
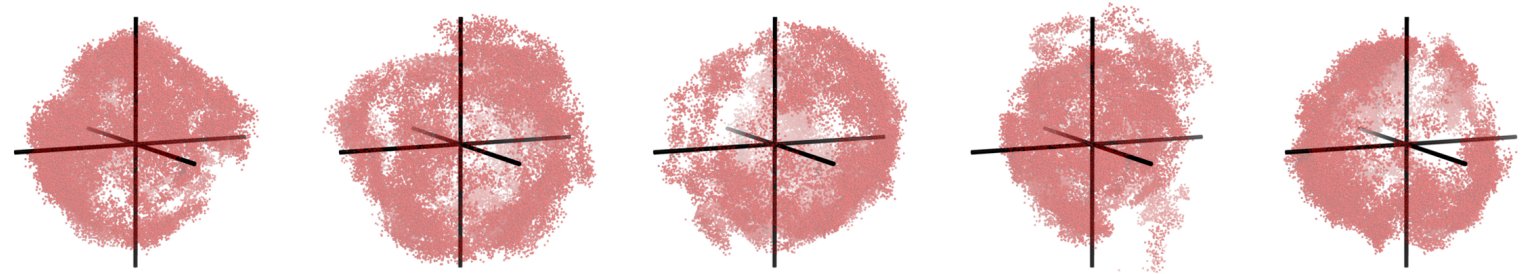

Figure S16.3: Regions explored by the substrate molecules in A3. For each replicate, a scatter plot is shown for each of the 5 substrate molecules, showing its center-of-mass coordinates (collected every 2 ps) as red dots. Only snapshots from productive interactions with the dendrimer (according to the criteria described in the main text) were considered. For each replicate, a coordinate system is shown with origin at the dendrimer center-ofmass. 
A4: Replicate 1
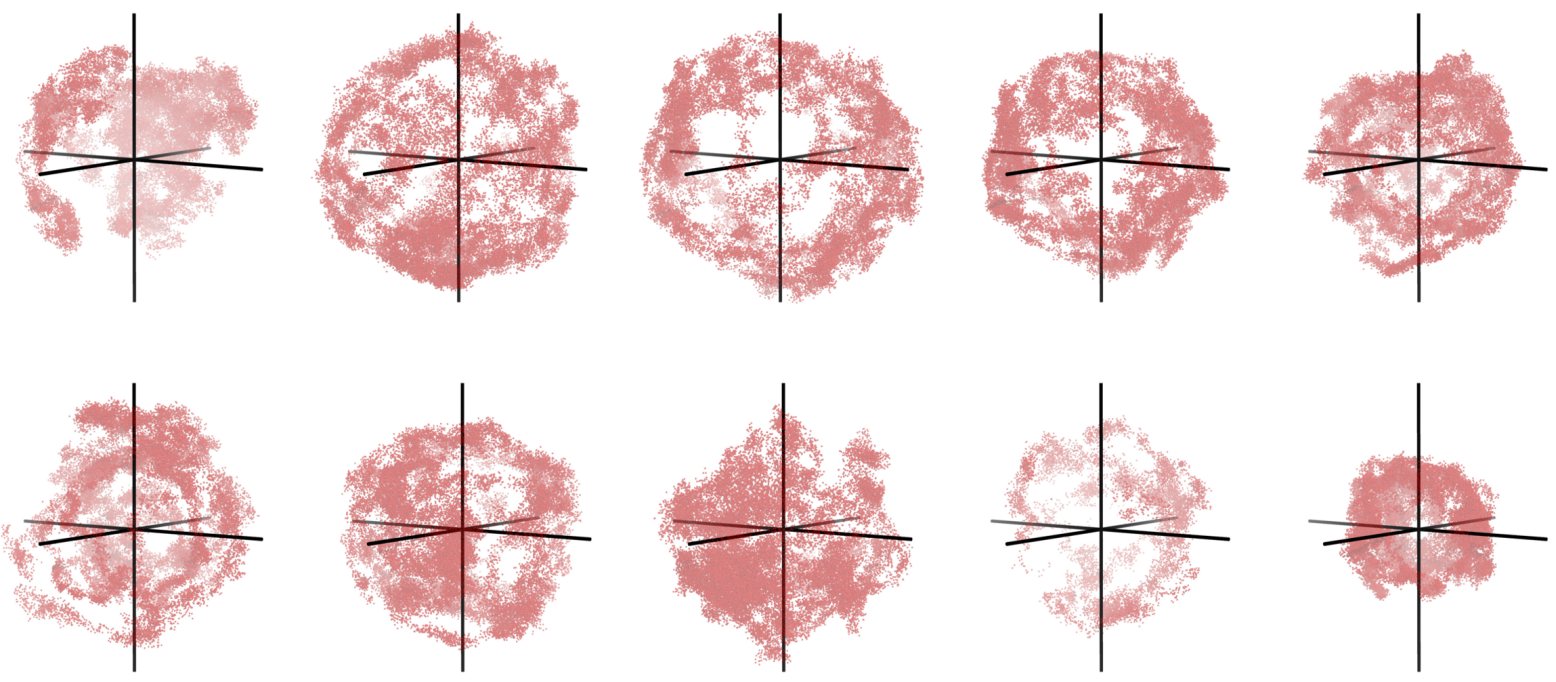

A4: Replicate 2
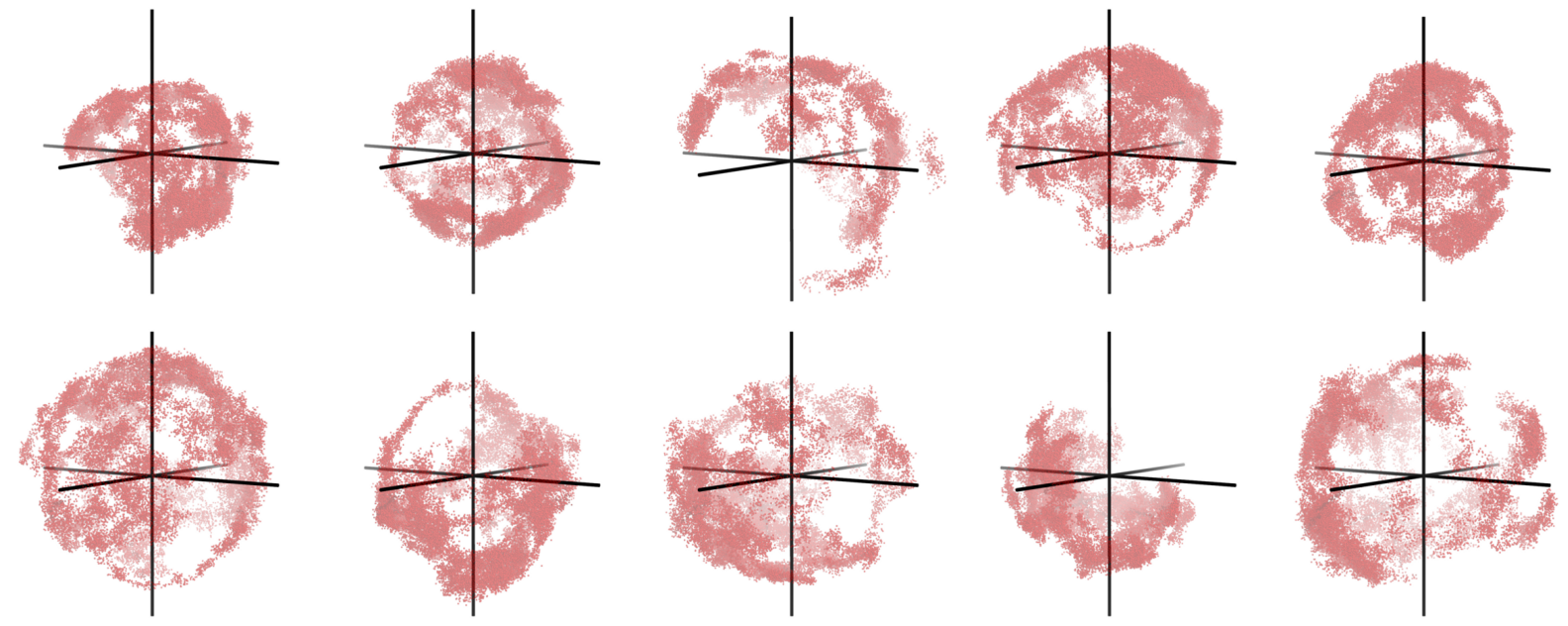
A4: Replicate 3
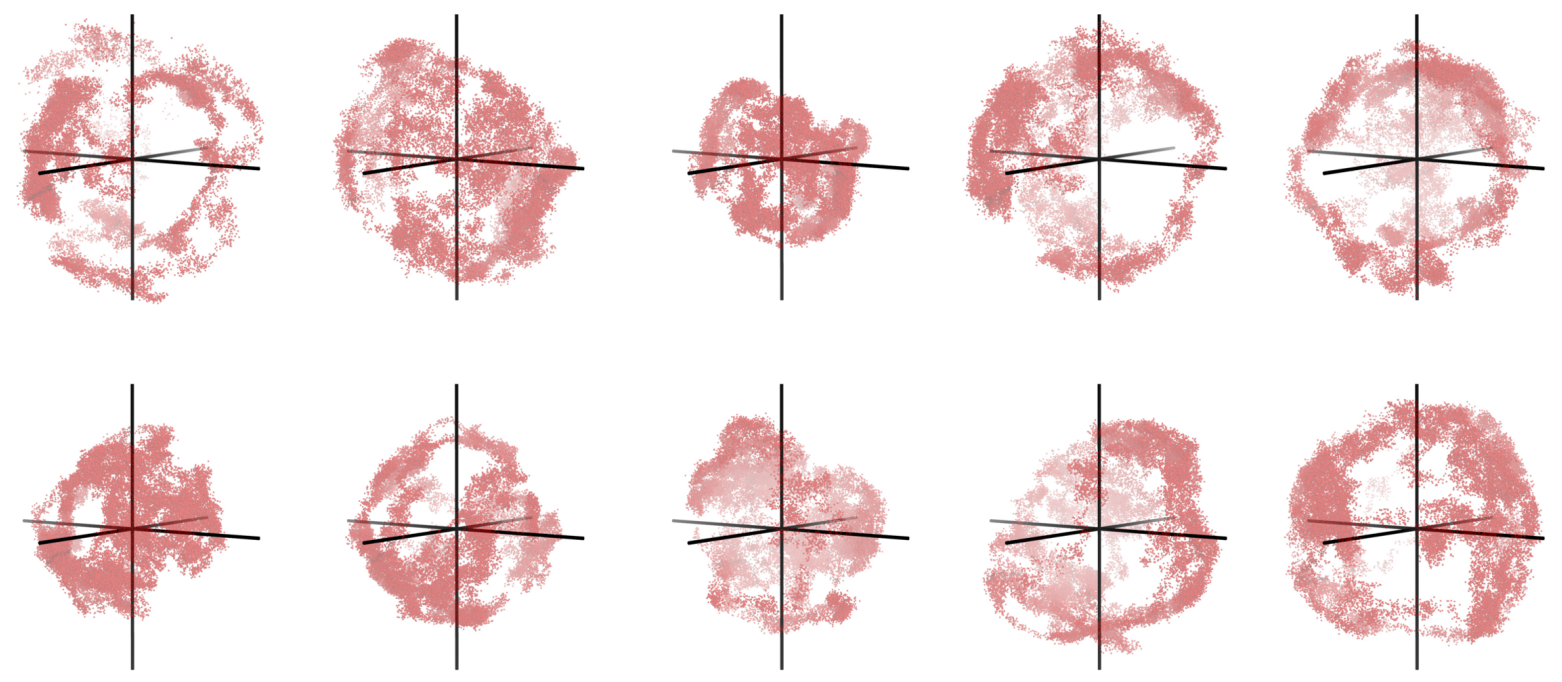

A4: Replicate 4
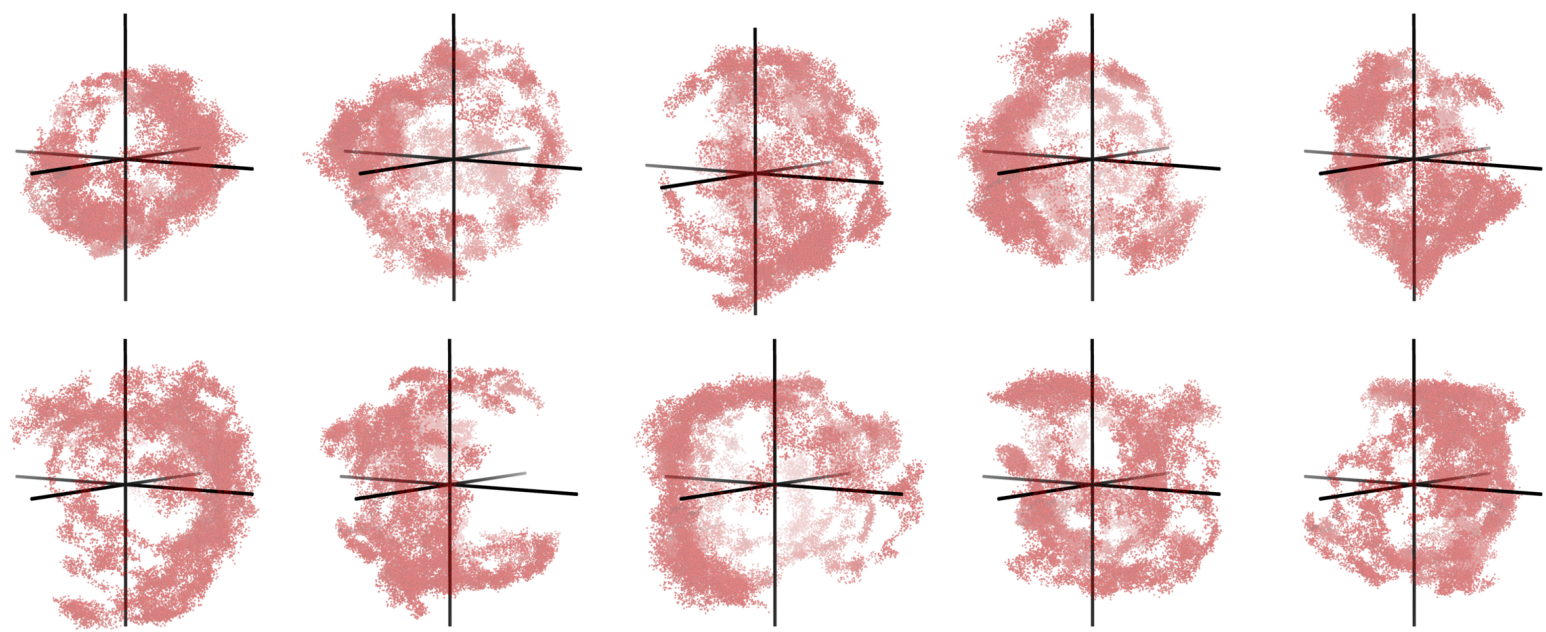


\section{A4: Replicate 5}
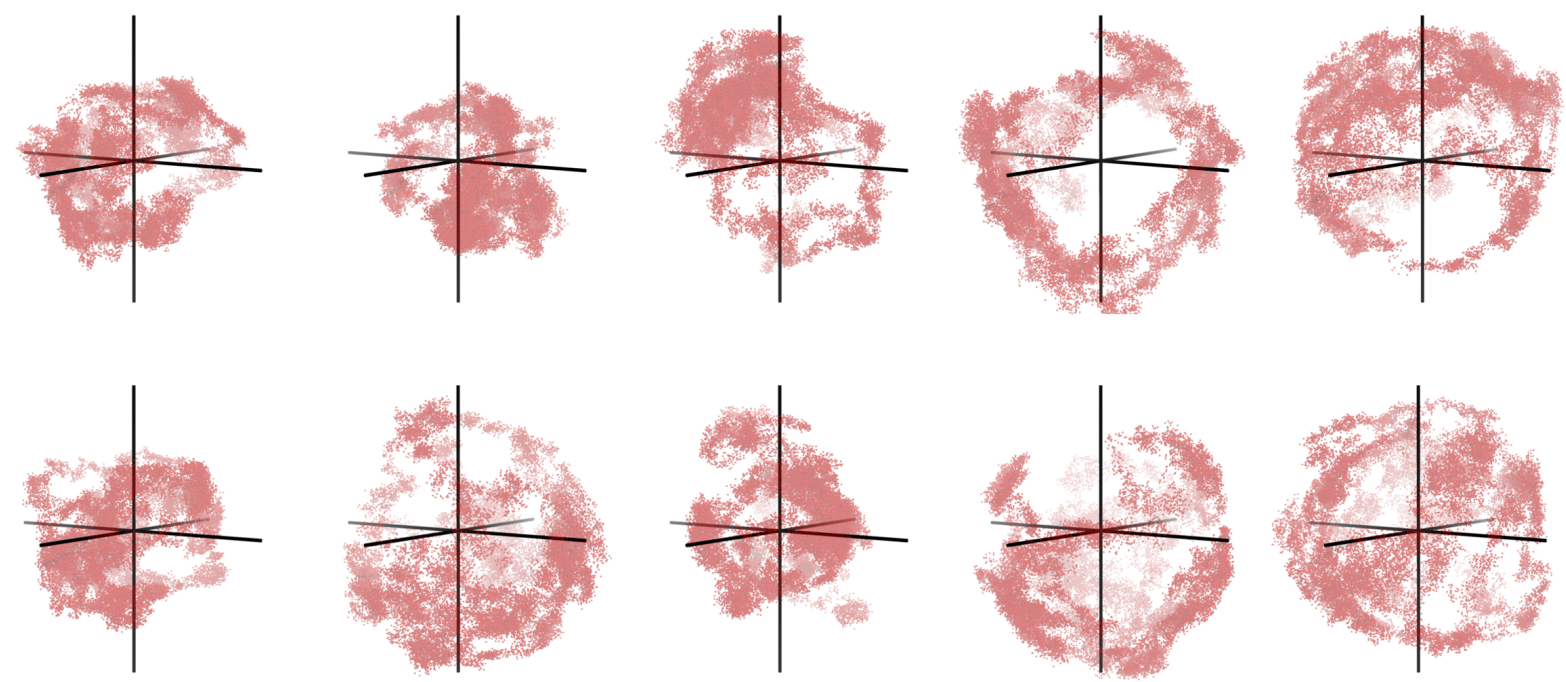

Figure S16.4: Regions explored by the substrate molecules in A4. For each replicate, a scatter plot is shown for each of the 10 substrate molecules, showing its center-of-mass coordinates (collected every 2 ps) as red dots. Only snapshots from productive interactions with the dendrimer (according to the criteria described in the main text) were considered. For each replicate, a coordinate system is shown with origin at the dendrimer center-of-mass. 


\section{References}

(1) Teixeira, V. H.; Vila-Viçosa, D.; Baptista, A. M.; Machuqueiro, M. Journal of Chemical Theory and Computation 2014, 10, 2176-2184.

(2) Bayly, C. I.; Cieplak, P.; Cornell, W.; Kollman, P. A. The Journal of Physical Chemistry $1993,97,10269-10280$.

(3) Schmid, N.; Eichenberger, A. P.; Choutko, A.; Riniker, S.; Winger, M.; Mark, A. E.; van Gunsteren, W. F. European Biophysics Journal 2011, 40, 843-856.

(4) Delort, E.; Nguyen-Trung, N.-Q.; Darbre, T.; Reymond, J.-L. The Journal of Organic Chemistry 2006, 71, 4468-4480.

(5) Eisenhaber, F.; Lijnzaad, P.; Argos, P.; Sander, C.; Scharf, M. Journal of Computational Chemistry 1995, 16, 273-284. 